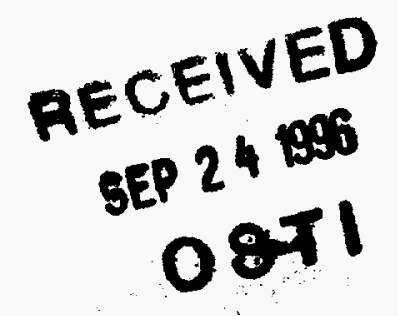

U.S. Departmenn of Energy
Portsmouth Site

Annual Environmental Data
for 1994
ES/ESH-65

POEF-3056
LOCKEED MARTNय

P(t)

A

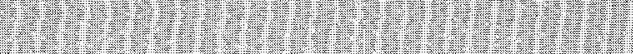

(C)

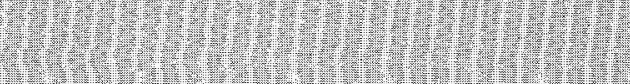

1.
LOCKHEED MARTIN ENERGY SYSTEMS, INC.

FOR TIE UNITED STATES

DEPARTMENT OF ENERGY

.

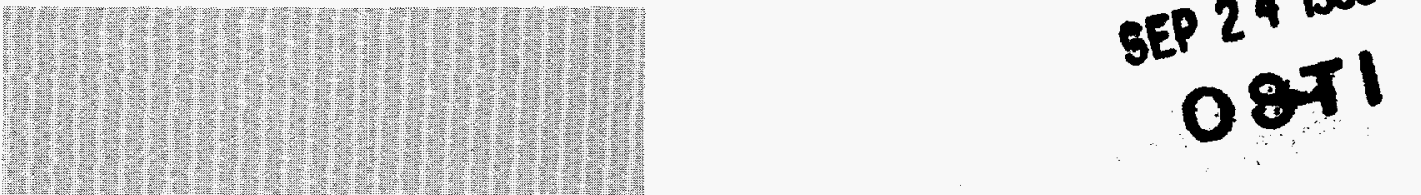

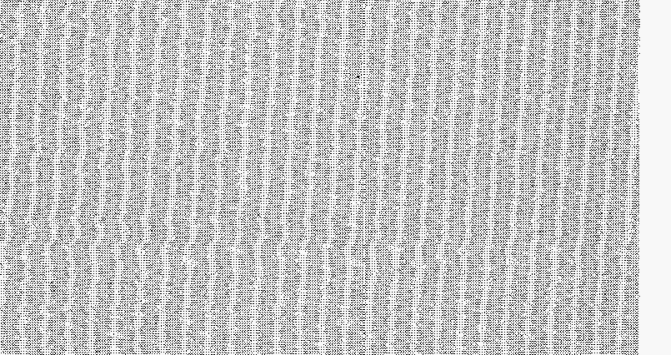

(1)
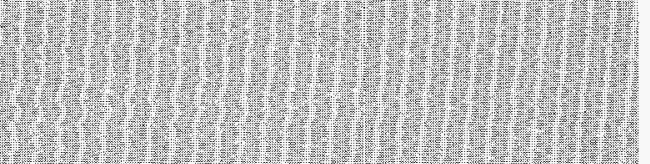

1.

\section{MANAGED BY}

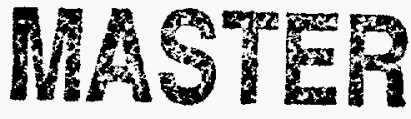

HH 
This report has been reproduced directly from the best available copy.

Available to DOE and DOE contractors from the Office of Scientific and Technical Information, P.O. Box 62, Oak Ridge, TN 37831; prices available from (615) 576-8401.

Available to the public from the National Technical Information Service, U.S. Department of Commerce, 5285 Port Royal Rd., Springfield, VA 22161.

This report was prepared as an account of work sponsored by an agency of the United States Government. Neither the United States nor any agency thereof, nor any of their employees, makes any warranty, express or implied, or assumes any legal liability or responsibility for the accuracy, completeness, or usefulness of any information, apparatus, product, or process disclosed, or represents that its use would not infringe privately owned rights. Reference herein to any specific commercial product, process, or senvice by trade name, trademark, manufacturer, or otherwise, does not necessarily constitute or imply its endorsement, recommendation, or favoring by the United States Government or any agency thereof. The views and opinions of authors expressed herein do not necessarily state or reflect those of the United States Government or any agency thereof. 


\section{DISCLAIMER}

This report was prepared as an account of work sponsored by an agency of the United States Government. Neither the United States. Government nor any agency thereof, nor any of their employees, makes any warranty, express or implied, or assumes any legal liability or responsibility for the accuracy, completeness, or usefulness of any information, apparatus, product, or process disclosed, or represents that its use would not infringe privately owned rights. Reference herein to any specific commercial product, process, or service by trade name, trademark, manufacturer, or otherwise does not necessarily constitute or imply its endorsement, recommendation, or favoring by the United States Government or any agency thereof. The views and opinions of authors expressed herein do not necessarily state or reflect those of the United States Government or any agency thereof. 


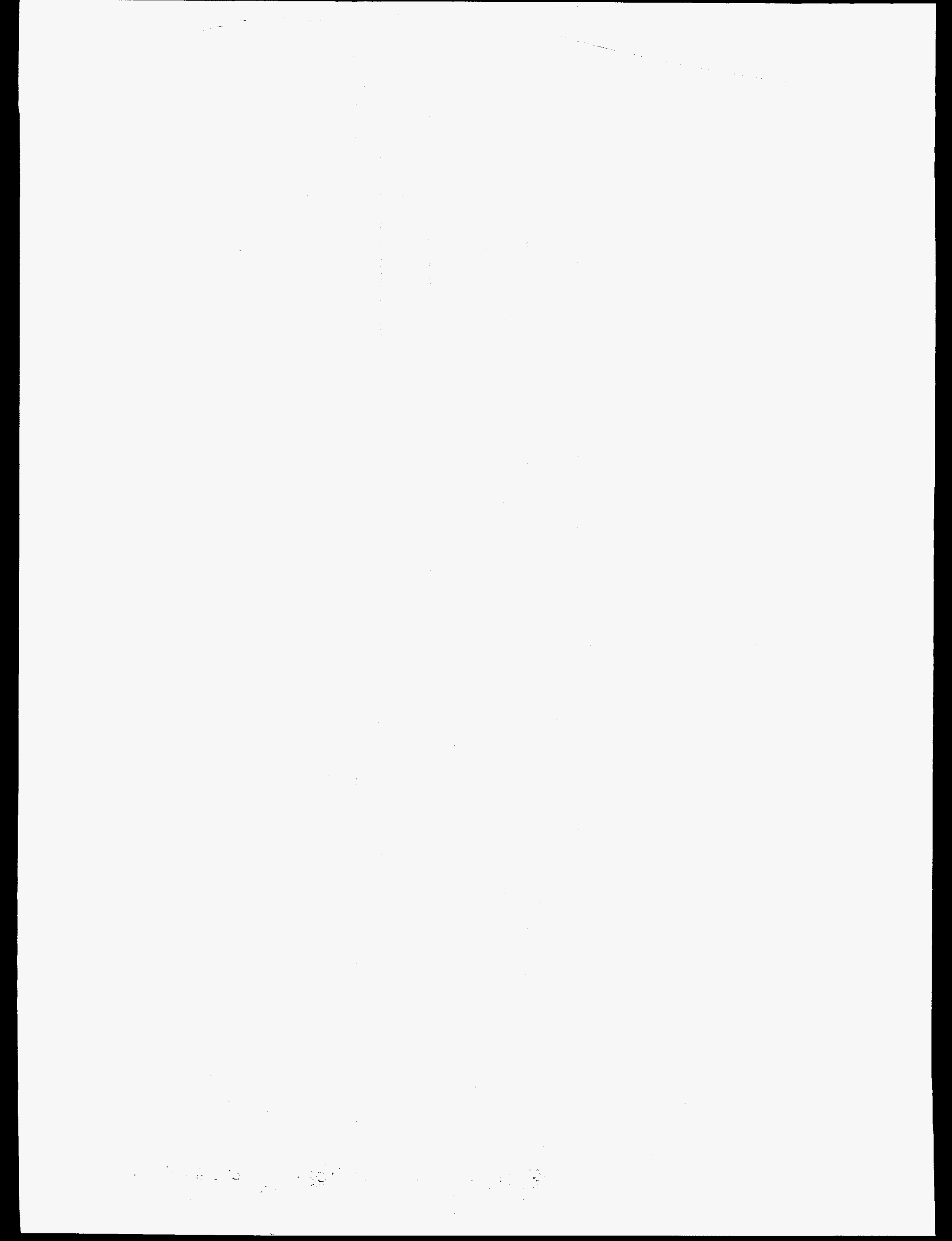




\title{
U.S. DEPARTMENT OF ENERGY PORTSMOUTH SITE ANNUAL ENVIRONMENTAL DATA FOR 1994
}

\author{
Project director \\ D. W. Frazier
}

Project coordinator

D. C. West

Technical coordinator

S. C. Newman/P. A. Sperling

\section{Coordinating editor}

C. M. Horak

Date Published: March 1996

\section{Prepared by}

Environmental, Safety, and Health Compliance and Environmental Management staffs LOCKHEED MARTIN ENERGY SYSTEMS, INC.

P.O. Box 2008

Oak Ridge, Tennessee 37831-6285 and the

Environmental Management Division

Portsmouth Site

LOCKHEED MARTIN ENERGY SYSTEMS, INC.

P.O. Box 628

Piketon, Ohio 45661

$$
\text { for the }
$$

U.S. DEPARTMENT OF ENERGY

under contract No. DE-AC05-84OR21400

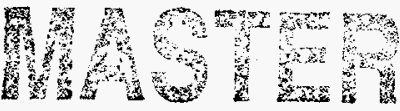





\section{DISCLAIMER}

Portions of this document may be illegible in electronic image products. Images are produced from the best available original document. 


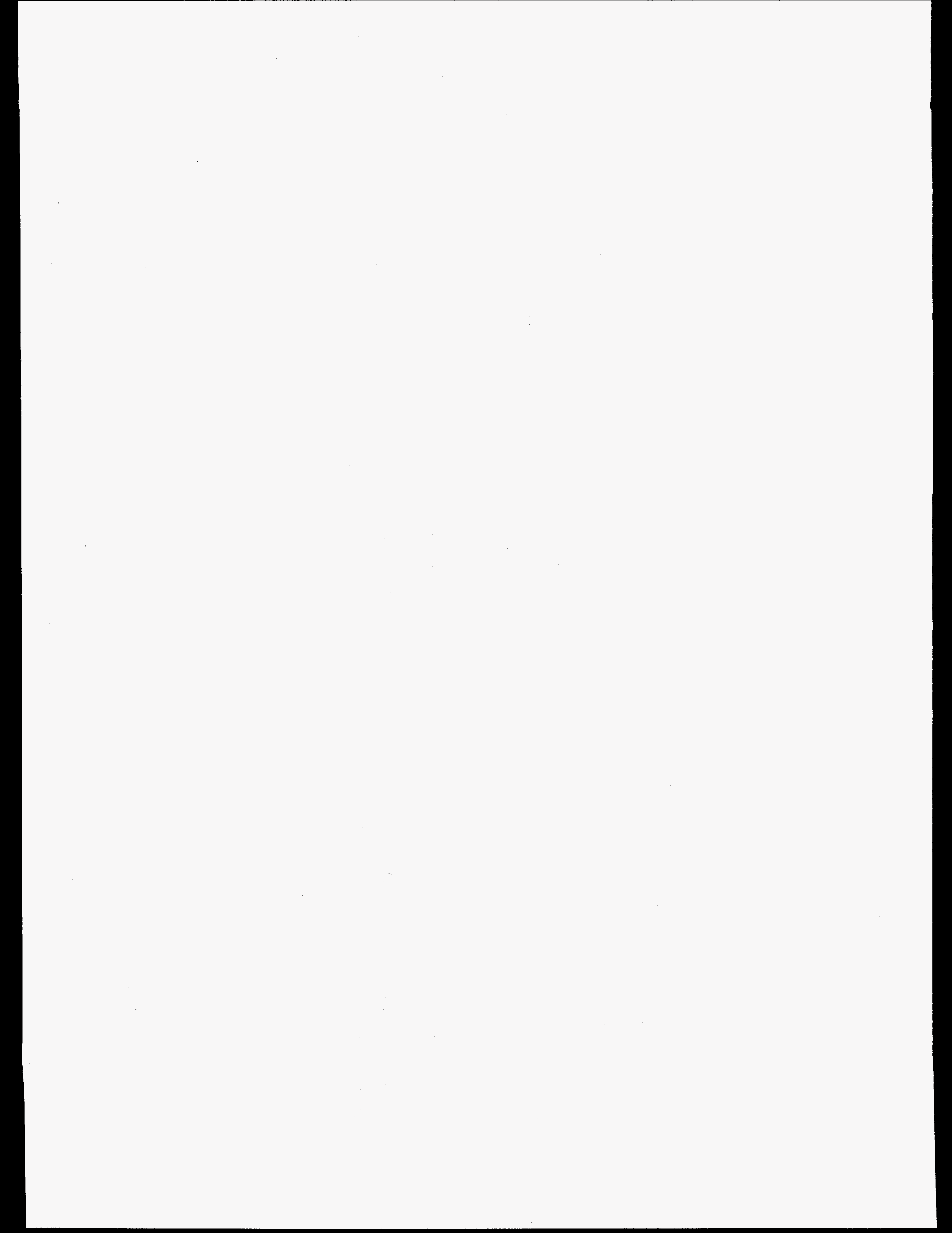




\section{Contents}

Page

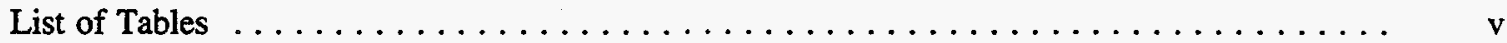

Acronyms and Abbreviations $\ldots \ldots \ldots \ldots \ldots \ldots \ldots \ldots \ldots \ldots \ldots \ldots \ldots \ldots \ldots \ldots$ vii

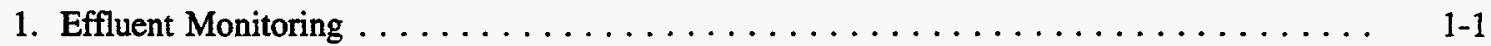

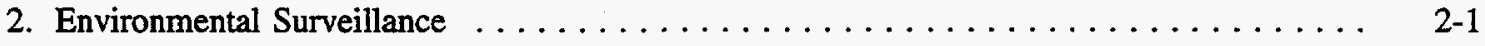

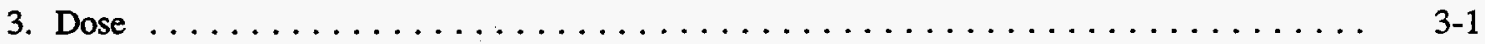

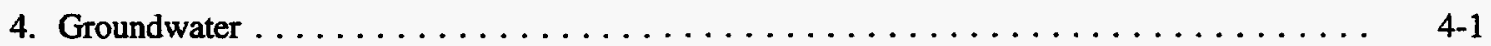





\section{List of Tables}

Table

Page

$1.1 \quad$ Radiological releases to air $(50-m$ vents) in $1994 \ldots \ldots \ldots \ldots \ldots \ldots \ldots \ldots \ldots$

1.2 Radiological releases to air $(20-\mathrm{m}$ vents) in $1994 \ldots \ldots \ldots \ldots \ldots \ldots \ldots \ldots \ldots$

$1.3 \quad$ Radiological releases to air $(3-m$ vent) in $1994 \ldots \ldots \ldots \ldots \ldots \ldots \ldots \ldots \ldots \ldots$

1.4 Superfund Amendments and Reauthorization Act, Section 313, releases

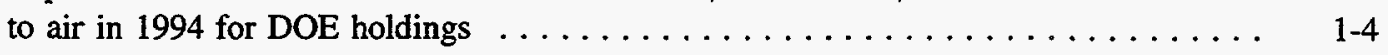

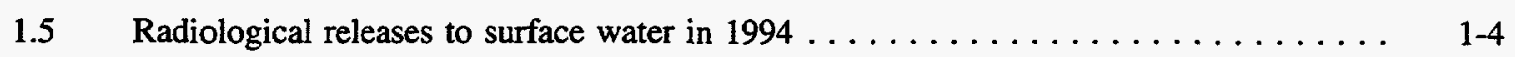

1.6 Uranium concentrations in water samples for $1994 \ldots \ldots \ldots \ldots \ldots \ldots \ldots \ldots$

1.7 Radioactivity concentrations in water samples for $1994 \ldots \ldots \ldots \ldots \ldots \ldots \ldots$

1.8 Portsmouth National Pollutant Discharge Elimination System permit

1.9 NPDES discharges and compliance rates for $1994 \ldots \ldots \ldots \ldots \ldots \ldots \ldots \ldots$

1.10 Superfund Amendments and Reauthorization Act, Section 313, releases to

surface water during 1994 for DOE holdings $\ldots \ldots \ldots \ldots \ldots \ldots \ldots \ldots \ldots \ldots \ldots \ldots$

2.1 Quarterly external gamma radiation levels $(\mu \mathrm{rad} / \mathrm{hour})$ for $1994 \ldots \ldots \ldots \ldots \ldots$ 2-3

2.2 Airborne gross alpha radiation concentrations $\left(\mathrm{pCi} / \mathrm{m}^{3}\right)$ for $1994 \ldots \ldots \ldots \ldots \ldots$

2.3 Airborne gross beta-gamma radiation concentrations $\left(\mathrm{pCi} / \mathrm{m}^{3}\right)$ for $1994 \ldots \ldots \ldots$ 2-5

2.4 Airborne gross gaseous fluoride concentrations $\left(\mu \mathrm{g} / \mathrm{m}^{3}\right)$ for $1994 \ldots \ldots \ldots \ldots \ldots$ 2-6

3.1 Committed effective dose rate conversion factors $\ldots \ldots \ldots \ldots \ldots \ldots \ldots \ldots$

3.2 Release point parameters and receptor locations used in dose calculations . . . . . 3-3

3.3 Activities ( $\mathrm{Ci})$, solubilities, and $\mathrm{AMAD}(\mu \mathrm{m})$ of radionuclides released from

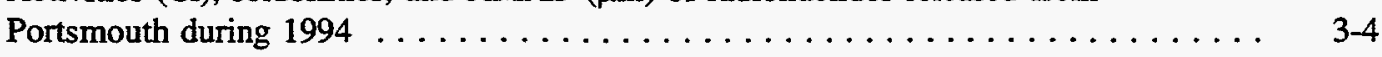

3.4 Predicted radiation doses from airborne releases at the Portsmouth

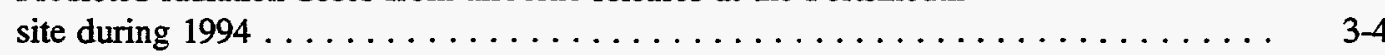

3.5 Predicted radiation doses from waterborne releases at the Portsmouth

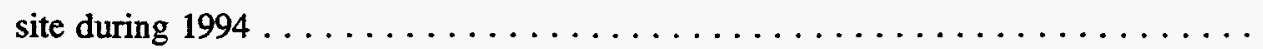

4.1 Portsmouth groundwater monitoring for 1994: trichloroethylene results for the $\mathrm{X}-701 \mathrm{~B}$ holding pond $\ldots \ldots \ldots \ldots \ldots \ldots \ldots \ldots \ldots \ldots \ldots \ldots \ldots \ldots \ldots \ldots \ldots \ldots$ 
4.2 Portsmouth groundwater monitoring for 1994: radionuclide results for the

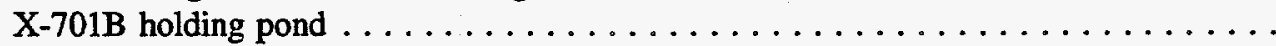

4.3 Portsmouth groundwater monitoring for 1994: results for volatile organic compounds

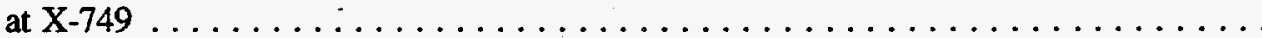

4.4 Portsmouth groundwater monitoring for 1994: results for radionuclide parameters

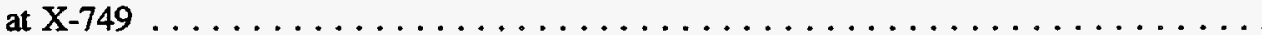

4.5 Portsmouth groundwater monitoring for 1994: results for volatile organic compounds at the $\mathrm{X}-231 \mathrm{~B}$ oil biodegradation plot $\ldots \ldots \ldots \ldots \ldots \ldots \ldots \ldots \ldots \ldots$

4.6 Portsmouth groundwater monitoring for 1994: results for radionuclide parameters at $\mathrm{X}-231 \mathrm{~B}$

4.7 Portsmouth groundwater monitoring for 1994: results for volatile organic compounds at X-616

4.8 Portsmouth groundwater monitoring for 1994: dissolved and total chromium results for X-616

4.9 Portsmouth groundwater monitoring for 1994: surface water monitoring results for

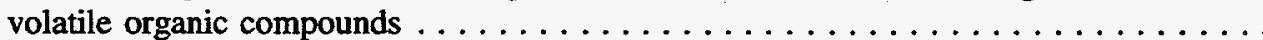

4.10 Portsmouth groundwater monitoring for 1994: surface water monitoring results for radionuclide parameters

4.11 Portsmouth groundwater monitoring for 1994: results for trichloroethylene at $\mathrm{X}-701 \mathrm{C}$

4.12 Portsmouth groundwater monitoring for 1994: results for radionuclide parameters at $\mathrm{X}-701 \mathrm{C}$ 


\section{Acronyms and Abbreviations}

ADI

AMAD

Bkg

Btu

${ }^{\circ} \mathrm{C}$

CDI

CFC

$\mathrm{Ci}$

$\mathrm{cm}$

$\mathrm{cm}^{2}$

$\mathrm{cm}^{3}$

$\mathrm{CO}$

col.

$\mathrm{Cr}$

DCA

DCE

DCG

DOE

EDE

ENE

$\mathrm{ft}$

g

gal

$\mathrm{kg}$

$\mathrm{km}$

L

$\mathrm{lb}$

m

$\mathrm{m}^{3}$

$\mathbf{M}$

$\max$

$\mu \mathrm{g}$

$\mu \mathrm{rad}$

$\mu \mathrm{Ci}$

$\mu \mathrm{g}$

$\mu \mathrm{m}$

$\mathrm{mCi}$

MCL

MCLG

acceptable daily intake

activity median aerodynamic diameter

background

British thermal unit

degrees celsius

chronic daily intake

chlorofluorocarbon

curie

centimeter

square centimeter

cubic centimeter

carbon monoxide

colony

chromium

dichloroethane

dichloroethene

derived concentration guide

U.S. Department of Energy

effective dose equivalent

east-northeast

feet

gram

gallon

kilogram

kilometer

liter

pound

meter

cubic meter

million

maximum

microgram

microrad

microcurie

microgram

micrometer

millicurie

maximum contaminant level

maximum contaminant level goal 


\begin{tabular}{|c|c|}
\hline $\begin{array}{l}\mathrm{mg} \\
\mathrm{Mgd} \\
\mathrm{min} \\
\mathrm{mL} \\
\text { mrem }\end{array}$ & $\begin{array}{l}\text { milligram } \\
\text { million gallons per day } \\
\text { minimum } \\
\text { milliliter } \\
\text { millirem }\end{array}$ \\
\hline NA & not applicable \\
\hline NPDES & National Pollutant Discharge Elimination System \\
\hline $\begin{array}{l}\mathrm{Pa} \\
\mathrm{PCB} \\
\mathrm{pCi}\end{array}$ & $\begin{array}{l}\text { protactinium } \\
\text { polychlorinated biphenyl } \\
\text { picocurie }\end{array}$ \\
\hline $\begin{array}{l}\text { s } \\
\text { SARA } \\
\text { SU }\end{array}$ & $\begin{array}{l}\text { second } \\
\text { Superfund Amendments and Reauthorization Act } \\
\text { standard unit }\end{array}$ \\
\hline $\begin{array}{l}\text { Tc } \\
\text { TCA } \\
\text { TCE } \\
\text { Th }\end{array}$ & $\begin{array}{l}\text { technetium } \\
\text { trichloroethane } \\
\text { trichloroethylene } \\
\text { thorium }\end{array}$ \\
\hline $\begin{array}{l}\mathrm{U} \\
\mathrm{USEC}\end{array}$ & $\begin{array}{l}\text { uranium } \\
\text { United States Enrichment Corporation }\end{array}$ \\
\hline
\end{tabular}




\section{Introduction}

Environmental monitoring on the Portsmouth Gaseous Diffusion Plant site and its surroundings is conducted throughout the year. Monitoring ensures that the site is a safe place to work, that plant operations do not adversely affect neighboring communities, and that activities comply with federal and state regulations.

This document is a compilation of the environmental monitoring data for calendar year 1994 and is intended as a tool for analysts in environmental monitoring, environmental restoration, and other related disciplines. The data in this document form the basis for the summary information in the Portsmouth Site Annual Environmental Report for 1994 (ES/ESH-63, POEF-3055). 

1. Effluent Monitoring 

Table 1.1. Radiological releases to air (50-m vents) in 1994

\begin{tabular}{llcccccc}
\hline \multicolumn{1}{c}{ Month } & $\begin{array}{c}{ }^{234} \mathrm{U} \\
(\mathrm{mCi})\end{array}$ & $\begin{array}{c}{ }^{235} \mathrm{U} \\
(\mathrm{mCi})\end{array}$ & $\begin{array}{c}{ }^{236} \mathrm{U} \\
(\mathrm{mCi})\end{array}$ & $\begin{array}{c}{ }^{238} \mathrm{U} \\
(\mathrm{mCi})\end{array}$ & $\begin{array}{c}\text { Uranium } \\
(\mathrm{kg})\end{array}$ & $\begin{array}{c}{ }^{99} \mathrm{Tc} \\
(\mathrm{mCi})\end{array}$ & $\begin{array}{c}\text { Uranium } \\
\text { daughters } \\
(\mathrm{mCi})\end{array}$ \\
\hline January & 6.00 & 0.166 & 0.000526 & 0.038 & 0.194 & 7.13 & 0.243 \\
February & 1.86 & 0.050 & 0.000169 & 0.011 & 0.056 & 8.67 & 0.072 \\
March & 2.73 & 0.062 & 0.000173 & 0.020 & 0.090 & 16.80 & 0.102 \\
April & 4.28 & 0.082 & 0.000174 & 0.040 & 0.160 & 26.81 & 0.162 \\
May & 3.85 & 0.076 & 0.000183 & 0.029 & 0.124 & 13.60 & 0.134 \\
June & 1.99 & 0.049 & 0.000106 & 0.048 & 0.168 & 8.76 & 0.145 \\
July & 2.12 & 0.050 & 0.000109 & 0.044 & 0.157 & 13.05 & 0.138 \\
August & 3.14 & 0.071 & 0.000164 & 0.052 & 0.191 & 9.27 & 0.175 \\
September & 3.02 & 0.078 & 0.000192 & 0.089 & 0.304 & 4.33 & 0.255 \\
October & 1.24 & 0.034 & 0.000094 & 0.044 & 0.148 & 5.00 & 0.122 \\
November & 0.84 & 0.020 & 0.000045 & 0.019 & 0.066 & 2.48 & 0.058 \\
December & 0.65 & 0.028 & 0.000048 & 0.018 & 0.064 & 4.91 & 0.054 \\
\multicolumn{1}{c}{ Misc. } & 0.49 & 0.012 & 0.000028 & 0.012 & 0.042 & 1.06 & 0.036 \\
\hline \multicolumn{1}{c}{ Total } & $3.22 \mathrm{E}+01$ & $7.69 \mathrm{E}-01$ & $2.01 \mathrm{E}-03$ & $4.64 \mathrm{E}-01$ & $1.76 \mathrm{E}+00$ & $1.22 \mathrm{E}+02$ & $1.70 \mathrm{E}+00$ \\
\hline
\end{tabular}

Table 1.2. Radiological releases to air (20-m vents) in 1994

\begin{tabular}{lccccccc}
\hline \multicolumn{1}{c}{ Month } & $\begin{array}{c}{ }^{234} \mathrm{U} \\
(\mathrm{mCi})\end{array}$ & $\begin{array}{c}{ }^{235} \mathrm{U} \\
(\mathrm{mCi})\end{array}$ & $\begin{array}{c}{ }^{236} \mathrm{U} \\
(\mathrm{mCi})\end{array}$ & $\begin{array}{c}{ }^{238} \mathrm{U} \\
(\mathrm{mCi})\end{array}$ & $\begin{array}{c}\text { Uranium } \\
(\mathrm{kg})\end{array}$ & $\begin{array}{c}{ }^{99} \mathrm{Tc} \\
(\mathrm{mCi})\end{array}$ & $\begin{array}{c}\text { Uranium } \\
\text { daughters } \\
(\mathrm{mC})\end{array}$ \\
\hline January & 1.159 & 0.0076 & 0.00012 & 0.092 & 0.280 & 1.1 & 0.191 \\
February & 1.920 & 0.0989 & 0.00203 & 1.050 & 3.204 & 1.5 & 2.198 \\
March & 0.418 & 0.0173 & 0.00031 & 0.154 & 1.471 & 1.7 & 0.325 \\
April & 0.357 & 0.0165 & 0.00028 & 0.145 & 0.444 & 1.4 & 0.307 \\
May & 1.150 & 0.0075 & 0.00016 & 0.084 & 0.257 & 1.0 & 0.176 \\
June & 0.405 & 0.0134 & 0.00023 & 0.114 & 0.349 & 1.5 & 0.241 \\
July & 0.177 & 0.0067 & 0.00009 & 0.047 & 0.145 & 1.1 & 0.101 \\
August & 0.149 & 0.0070 & 0.00018 & 0.092 & 0.280 & 1.3 & 0.191 \\
September & 0.152 & 0.0055 & 0.00010 & 0.050 & 0.154 & 1.5 & 0.106 \\
October & 0.112 & 0.0049 & 0.00010 & 0.052 & 0.160 & 1.2 & 0.109 \\
November & 0.098 & 0.0043 & 0.00009 & 0.047 & 0.142 & 1.3 & 0.097 \\
December & 0.195 & 0.0082 & 0.00013 & 0.064 & 0.185 & 1.5 & 0.135 \\
$\quad$ Misc. & 0.038 & 0.0020 & 0.00005 & 0.028 & 0.085 & 0.7 & 0.098 \\
\cline { 2 - 8 } Total & $4.33 \mathrm{E}-00$ & $2.00 \mathrm{E}-01$ & $3.87 \mathrm{E}-03$ & $2.02 \mathrm{E}+00$ & $6.17 \mathrm{E}+00$ & $1.69 \mathrm{E}+01$ & $4.24 \mathrm{E}+00$ \\
\hline
\end{tabular}


Table 1.3. Radiological releases to air (3-m vent) in 1994

\begin{tabular}{llcccccc}
\hline \multicolumn{1}{c}{ Month } & $\begin{array}{c}{ }^{234} \mathrm{U} \\
(\mathrm{mCi})\end{array}$ & $\begin{array}{c}{ }^{235} \mathrm{U} \\
(\mathrm{mCi})\end{array}$ & $\begin{array}{c}{ }^{236} \mathrm{U} \\
(\mathrm{mCi})\end{array}$ & $\begin{array}{c}{ }^{238} \mathrm{U} \\
(\mathrm{mCi})\end{array}$ & $\begin{array}{c}\text { Uranium } \\
(\mathrm{kg})\end{array}$ & $\begin{array}{c}{ }^{99} \mathrm{Tc} \\
(\mathrm{mCi})\end{array}$ & $\begin{array}{c}\text { Uranium } \\
\text { daughters } \\
(\mathrm{mC})\end{array}$ \\
\hline January & 0.000 & 0.0000 & 0.000000 & 0.0000 & 0.0000 & 0.0000 & 0.0000 \\
February & 0.000 & 0.0000 & 0.000000 & 0.0000 & 0.0000 & 0.0000 & 0.0000 \\
March & 0.000 & 0.0000 & 0.000000 & 0.0000 & 0.0000 & 0.0000 & 0.0000 \\
April & 0.000 & 0.0000 & 0.000000 & 0.0000 & 0.0000 & 0.0000 & 0.0000 \\
May & 0.000 & 0.0000 & 0.000000 & 0.0000 & 0.0000 & 0.0000 & 0.0000 \\
June & 0.000 & 0.0000 & 0.000000 & 0.0000 & 0.0000 & 0.0000 & 0.0000 \\
July & 0.000 & 0.0000 & 0.000000 & 0.0000 & 0.0000 & 0.0000 & 0.0000 \\
August & 0.000 & 0.0000 & 0.000000 & 0.0000 & 0.0000 & 0.0000 & 0.0000 \\
September & 0.000 & 0.0000 & 0.000000 & 0.0000 & 0.0000 & 0.0000 & 0.0000 \\
October & 0.000 & 0.0000 & 0.000000 & 0.0000 & 0.0000 & 0.0000 & 0.0000 \\
November & 0.000 & 0.0000 & 0.000000 & 0.0000 & 0.0000 & 0.0000 & 0.0000 \\
December & 0.000 & 0.0000 & 0.000000 & 0.0000 & 0.0000 & 0.0000 & 0.0000 \\
$\quad$ Misc. & 0.000 & 0.0000 & 0.000000 & 0.0000 & 0.0000 & 0.0000 & 0.0000 \\
\hline \multicolumn{1}{c}{ Total } & $0.00 \mathrm{E}+00$ & $0.00 \mathrm{E}+00$ & $0.00 \mathrm{E}+00$ & $0.00 \mathrm{E}+00$ & $0.00 \mathrm{E}+00$ & $0.00 \mathrm{E}+00$ & $0.00 \mathrm{E}+00$ \\
\hline
\end{tabular}

Table 1.4. Superfund Amendments and Reauthorization Act, Section 313, releases to air in 1994 for DOE holdings

\begin{tabular}{lccc}
\hline Chemical name & $\begin{array}{c}\text { Type of } \\
\text { environmental } \\
\text { release }\end{array}$ & $\begin{array}{c}\text { Released quantity } \\
(\mathrm{lb} / \mathrm{kg})\end{array}$ & Major release sources \\
\hline Hydrogen fluoride & Stack & $1600 / 726$ & Highly enriched uranium refeed system \\
\hline
\end{tabular}

Table 1.5. Radiological releases to surface water in 1994

\begin{tabular}{ccccccccc}
\hline $\begin{array}{c}\text { NPDES } \\
\text { outfall }\end{array}$ & $\begin{array}{c}{ }^{234} \mathrm{U} \\
(\mathrm{mCi})\end{array}$ & $\begin{array}{c}{ }^{235} \mathrm{U} \\
(\mathrm{mCi})\end{array}$ & $\begin{array}{c}{ }^{236} \mathrm{U} \\
(\mathrm{mCi})\end{array}$ & $\begin{array}{c}{ }^{238} \mathrm{U} \\
(\mathrm{mCi})\end{array}$ & $\begin{array}{c}\text { Total } \\
\text { uranium } \\
(\mathrm{kg})\end{array}$ & $\begin{array}{c}{ }^{99} \mathrm{Tc} \\
(\mathrm{mCi})\end{array}$ & $\begin{array}{c}\text { Uranium } \\
\text { daughters } \\
(\mathrm{mCi})\end{array}$ & $\begin{array}{c}\text { Total } \\
\text { flow } \\
(\mathrm{Mgal})\end{array}$ \\
\hline 012 & 0.45 & 0.024 & 0.00069 & 0.00096 & 0.604 & 0.0 & 0.038 & 134 \\
013 & 0.46 & 0.028 & 0.00071 & 0.00099 & 0.622 & 0.0 & 0.039 & 105 \\
\hline Total & 0.91 & 0.052 & 0.0014 & 0.00195 & 1.206 & 0.0 & 0.077 & 239 \\
\hline
\end{tabular}

"National Pollutant Discharge Elimination System. 
Table 1.6. Uranium concentrations in water samples for 1994

\begin{tabular}{cccccc}
\hline \multirow{2}{*}{$\begin{array}{c}\text { NPDES }^{a} \\
\text { outfall }\end{array}$} & $\begin{array}{c}\text { Number of } \\
\text { samples }\end{array}$ & $\begin{array}{c}\text { Maximum } \\
(\mathrm{mg} / \mathrm{L})\end{array}$ & $\begin{array}{c}\text { Minimum } \\
(\mathrm{mg} / \mathrm{L})\end{array}$ & $\begin{array}{c}\text { Average } \\
(\mathrm{mg} / \mathrm{L})\end{array}$ & $\begin{array}{c}\text { Standard } \\
\text { deviation }\end{array}$ \\
\hline 012 & 12 & 0.002 & $<0.001$ & $<0.001$ & 0.000 \\
013 & 12 & 0.002 & $<0.001$ & $<0.001$ & 0.000 \\
\hline
\end{tabular}

${ }^{a}$ National Pollutant Discharge Elimination System.

Table 1.7. Radioactivity concentrations in water samples for 1994

\begin{tabular}{|c|c|c|c|c|c|c|}
\hline \multirow{3}{*}{$\begin{array}{c}\text { NPDES }^{a} \\
\text { outfall }\end{array}$} & \multirow{3}{*}{$\begin{array}{l}\text { Number of } \\
\text { samples }\end{array}$} & \multicolumn{5}{|c|}{ Concentration } \\
\hline & & \multirow{2}{*}{$\begin{array}{l}\text { Maximum } \\
(\mathrm{pCi} / \mathrm{L})\end{array}$} & \multirow{2}{*}{$\begin{array}{c}\text { Minimum } \\
(\mathrm{pCi} / \mathrm{L})\end{array}$} & \multicolumn{2}{|c|}{ Average } & \multirow{2}{*}{$\begin{array}{l}\text { Standard } \\
\text { deviation }\end{array}$} \\
\hline & & & & $(\mathrm{pCi} / \mathrm{L})$ & $\left(\% \mathrm{DCG}^{b}\right)$ & \\
\hline \multicolumn{7}{|c|}{ Gross alpha } \\
\hline 012 & $12(12)^{c}$ & $<7$ & $<4$ & $<4.9$ & 0.98 & 0.720 \\
\hline 013 & $12(12)^{c}$ & $<9$ & $<4$ & $<5.7$ & 1.14 & 1.474 \\
\hline \multicolumn{7}{|c|}{${ }^{99} T c$} \\
\hline 012 & 12 & $<22$ & $<22$ & $<22.0$ & 0.22 & 0.0 \\
\hline 013 & 12 & $<22$ & $<22$ & $<22.0$ & 0.22 & 0.0 \\
\hline \multicolumn{7}{|c|}{ Gross beta } \\
\hline 012 & 12 & $<11$ & $<9$ & $<9.8$ & $\mathrm{NA}^{d}$ & 0.960 \\
\hline 013 & 12 & 12 & $<$ & $<9.9$ & NA & 1.06 \\
\hline
\end{tabular}

National Pollutant Discharge Elimination System.

Derived concentration guide.

Number in parentheses indicates the number of samples that were below the detection limit for gross alpha activity.

${ }^{d}$ Not applicable. 
Table 1.8. Portsmouth National Pollutant Discharge Elimination System permit summary for 1994

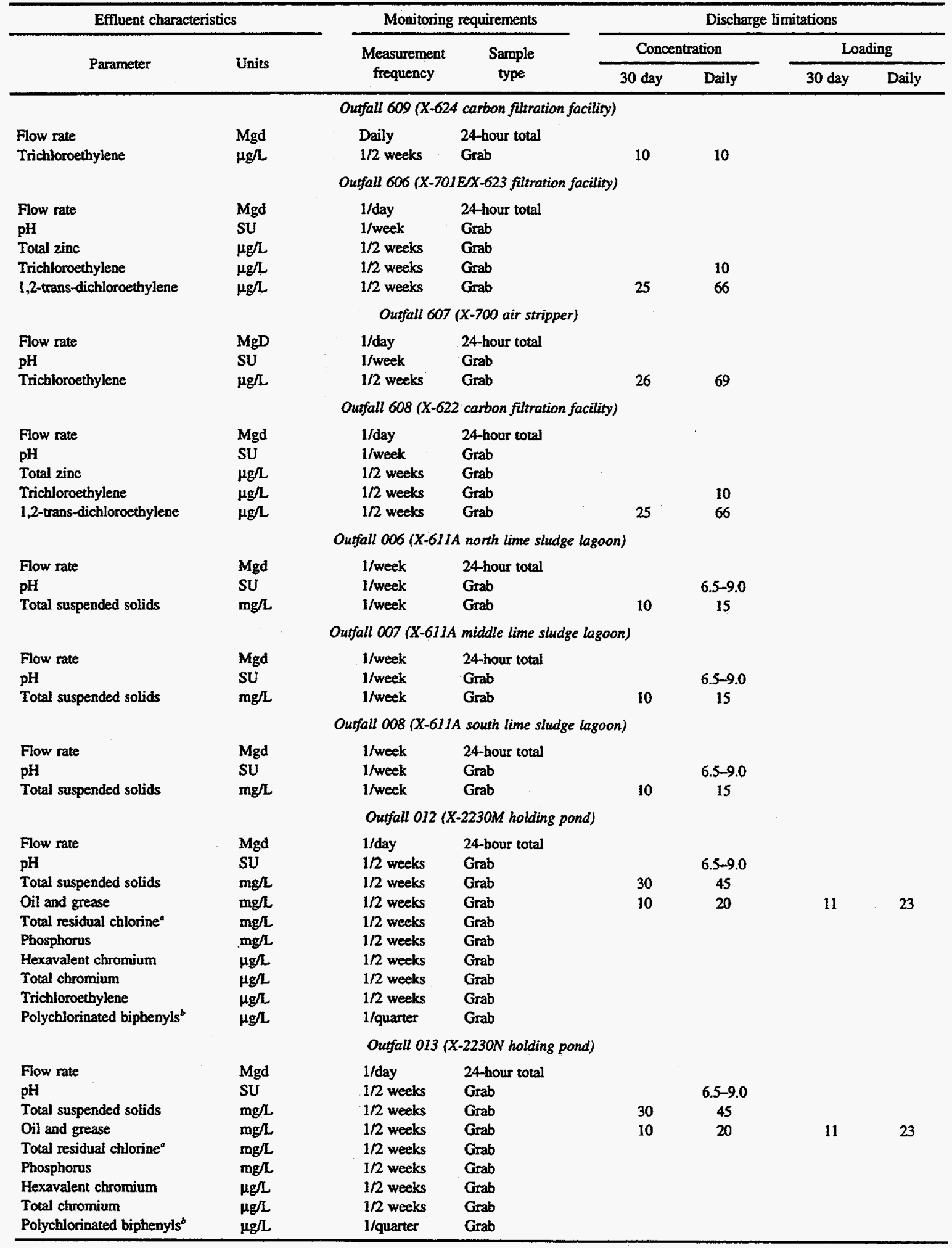

"Summer only.

No detectable polychlorinated biphenyls without a numerical limit specified. 
Table 1.9. 1994 NPDES discharge and compliance rates

\begin{tabular}{|c|c|c|c|c|c|c|c|}
\hline \multirow{2}{*}{ Parameter } & \multirow{2}{*}{$\begin{array}{c}\text { NPDES compliance } \\
\text { rate (\%) }\end{array}$} & \multirow{2}{*}{$\begin{array}{l}\text { Number of } \\
\text { samples }\end{array}$} & \multicolumn{3}{|c|}{ Concentrations/loadings } & \multirow{2}{*}{$\begin{array}{l}\text { Standard } \\
\text { deviation }\end{array}$} & \multirow{2}{*}{ Units } \\
\hline & & & Minimum & Maximum & Average & & \\
\hline \multicolumn{8}{|c|}{ Outfall 006 (X-61LA norh lime sludge lagoon) } \\
\hline Flow rate & $b$ & 22 & 0.01 & 0.84 & 0.28 & 0.32 & Mgd \\
\hline pH & $c$ & 5 & 8.10 & 9.50 & 9.10 & 0.50 & SU \\
\hline Total suspended solids & $c$ & 5 & 1.00 & 3.40 & 2.60 & 0.90 & $\mathbf{m g} / \mathbf{L}$ \\
\hline 30-day average & $c$ & 2 & 1.00 & 2.82 & 1.81 & 0.81 & $\mathbf{m g h}$ \\
\hline Polychlorinated biphenyls & $d$ & 1 & 1.00 & 1.00 & 1.00 & 0.00 & $\mu g / L$ \\
\hline \multicolumn{8}{|c|}{ Outfall 007 (X-611A middle lime sludge lagoon) } \\
\hline Flow rate & $b$ & 0 & 0.00 & 0.00 & 0.00 & 0.00 & Mgd \\
\hline $\mathbf{p H}$ & $c$ & 0 & 0.00 & 0.00 & 0.00 & 0.00 & $\mathbf{S U}$ \\
\hline Total suspended solids & $c$ & 0 & 0.00 & 0.00 & 0.00 & 0.00 & $\mathrm{mg} / \mathrm{L}$ \\
\hline 30-day average & $c$ & 0 & 0.00 & 0.00 & 0.00 & 0.00 & $\mathrm{mg} / \mathrm{L}$ \\
\hline Polychlorinated biphenyls & $d$ & 0 & 0.00 & 0.00 & 0.00 & 0.00 & $\mu g / L$ \\
\hline \multicolumn{8}{|c|}{ Outfall 008 (X-611A south lime siudge lagoon) } \\
\hline Flow rate & $b$ & 4 & 0.01 & 0.05 & 0.03 & 0.01 & Mgd \\
\hline pH & $c$ & 2 & 8.90 & 9.10 & 9.00 & 0.10 & SU \\
\hline Total suspended solids & $c$ & 2 & 2.20 & 3.80 & 3.00 & 0.80 & mg/ \\
\hline 30-day average & $c$ & 1 & 2.38 & 2.38 & 2.38 & 0.00 & $\mathrm{mg} / \mathrm{L}$ \\
\hline Polychlorinated biphenyls & $d$ & 1 & 1.00 & 1.00 & 1.00 & 0.00 & $\mu g / L$ \\
\hline \multicolumn{8}{|c|}{ Outfall 012 (X-2230M holding pond) } \\
\hline Flow rate & $b$ & 249 & 0.00 & 24.49 & 0.54 & 1.80 & Mgd \\
\hline $\mathrm{pH}$ & 100 & 27 & 7.00 & 8.20 & 7.70 & 0.30 & SU \\
\hline Total suspended solids & 96 & 25 & 1.00 & 61.00 & 14.14 & 13.37 & $\mathrm{mg} / \mathrm{L}$ \\
\hline 30-day average & 92 & 12 & 2.52 & 60.87 & 17.11 & 15.83 & $\mathrm{mg} / \mathrm{L}$ \\
\hline Oil and grease & 100 & 25 & 5.00 & 6.40 & 5.06 & 0.27 & $\mathrm{mg} / \mathrm{L}$ \\
\hline 30-day average & 100 & 12 & 5.00 & 5.58 & 5.05 & 0.16 & mgll \\
\hline daily loading & 100 & 25 & 0.00 & 46.76 & 7.15 & 11.13 & $\mathrm{~kg} / \mathrm{day}$ \\
\hline 30-day average loading & 100 & 12 & 0.01 & 17.99 & 6.50 & 6.36 & kg \\
\hline Phosphorus & $f$ & 25 & 0.02 & 0.09 & 0.04 & 0.02 & $\mathrm{mg} / \mathrm{L}$ \\
\hline Hexavalent chromium & $f$ & 25 & 0.01 & 0.01 & 0.01 & 0.00 & $\mu \mathrm{g} / \mathrm{L}$ \\
\hline Total chromium & $f$ & 25 & 0.02 & 14.00 & 1.50 & 3.16 & $\mu \mathrm{g} / \mathrm{L}$ \\
\hline Trichloroethylene & $f$ & 25 & 1.00 & 1.00 & 1.00 & 0.00 & $\mu \mathrm{g} / \mathrm{L}$ \\
\hline Total residual chlorine & $f$ & 13 & 0.02 & 0.02 & 0.02 & 0.00 & $\mathbf{m g} / \mathrm{L}$ \\
\hline Polychlorinated biphenyls & $d$ & 4 & 1.00 & 1.00 & 1.00 & 0.00 & $\mu g / L$ \\
\hline \multicolumn{8}{|c|}{ Outfall 013 (X-2230N holding pond) } \\
\hline Flow rate & $b$ & 249 & 0.00 & 16.87 & 0.42 & 1.39 & Mgd \\
\hline pH & 97 & 29 & 7.20 & 9.20 & 7.90 & 0.50 & SU \\
\hline Total suspended solids & 96 & 27 & 1.00 & 46.30 & 8.43 & 8.67 & $\mathrm{mg} / \mathrm{L}$ \\
\hline 30-day average & 100 & 12 & 2.16 & 19.80 & 8.30 & 5.04 & $\mathbf{m g} / \mathbf{L}$ \\
\hline Oil and grease & 100 & 27 & 5.00 & 6.60 & 5.06 & 0.30 & $\mathrm{mg} / \mathrm{L}$ \\
\hline 30-day average & 100 & 12 & 5.00 & 5.68 & 5.06 & 0.19 & $\mathrm{mg} / \mathrm{L}$ \\
\hline daily loading & 100 & 27 & 0.00 & 20.46 & 3.99 & 5.15 & $\mathrm{~kg} /$ day \\
\hline 30-day average loading & 100 & 12 & 0.22 & 15.60 & 4.04 & 4.13 & kg \\
\hline Phosphorus & $f$ & 27 & 0.02 & 0.15 & 0.06 & 0.03 & $\mathrm{mg} / \mathrm{L}$ \\
\hline Hexavalent chromium & $f$ & 27 & 0.01 & 0.01 & 0.01 & 0.00 & $\mu g / L$ \\
\hline Total chromium & $f$ & 27 & 0.02 & 14.00 & 1.16 & 3.02 & $\mu g / L$ \\
\hline Total residual chlorine & $f$ & 15 & 0.02 & 0.02 & 0.02 & 0.00 & $\mathbf{m g} / \mathrm{L}$ \\
\hline Polychlorinated biphenyls & $d$ & 4 & 1.00 & 1.00 & 1.00 & 0.00 & $\mu \mathrm{g} / \mathrm{L}$ \\
\hline \multicolumn{8}{|c|}{ Outfall 606 (X-701/X-623 carbon filtration facility) } \\
\hline Flow rate & $\boldsymbol{b}$ & 182 & 0.00 & 0.04 & 0.01 & 0.01 & Mgd \\
\hline pH & 100 & 13 & 6.90 & 8.60 & 7.40 & 0.60 & sU \\
\hline Trichloroethylene & 100 & 8 & 1.00 & 1.00 & 1.00 & 0.00 & $\mu g / L$ \\
\hline 1,2-trans-dichloroethylene & 100 & 8 & 1.00 & 40.00 & 5.88 & 12.90 & $\mu \mathrm{g} / \mathrm{L}$ \\
\hline 30-day average & 100 & 6 & 0.09 & 18.73 & 3.80 & 6.68 & $\mu g / L$ \\
\hline Total zinc & $f$ & 8 & 0.03 & 102.00 & 22.89 & 30.72 & $\mu g / L$ \\
\hline
\end{tabular}


Table 1.9 (continued)

\begin{tabular}{|c|c|c|c|c|c|c|c|c|}
\hline \multirow{2}{*}{ Parameter } & \multirow{2}{*}{\multicolumn{2}{|c|}{$\begin{array}{l}\text { NPDES compliance } \\
\text { rate }(\%)\end{array}$}} & \multirow{2}{*}{$\begin{array}{l}\text { Number of } \\
\text { samples }\end{array}$} & \multicolumn{3}{|c|}{ Concentrations/loadings } & \multirow{2}{*}{$\begin{array}{l}\text { Standard } \\
\text { deviation }\end{array}$} & \multirow{2}{*}{ Units } \\
\hline & & & & Minimum & Maximum & Average & & \\
\hline \multicolumn{9}{|c|}{ Outfall 607 (X-700 air stripper) } \\
\hline Flow rate & $b$ & & $\mathbf{0}$ & 0.00 & 0.00 & 0.00 & 0.00 & Mgd \\
\hline $\mathrm{pH}$ & $c$ & & 0 & 0.00 & 0.00 & 0.00 & 0.00 & SU \\
\hline Trichloroethylene & $c$ & & 0 & 0.00 & 0.00 & 0.00 & 0.00 & $\mu \mathrm{g} / \mathrm{L}$ \\
\hline 30-day average & $c$ & ' & 0 & 0.00 & 0.00 & 0.00 & 0.00 & $\mu g / L$ \\
\hline \multicolumn{9}{|c|}{ Outfall 608 (X-622 carbon filtration facility) } \\
\hline Flow rate & $\boldsymbol{b}$ & & 365 & 0.00 & 0.04 & 0.02 & 0.01 & Mgd \\
\hline pH & 100 & & 50 & 6.30 & 7.90 & 7.10 & 0.30 & SU \\
\hline Trichloroethylene & 100 & & 30 & 1.00 & 1.00 & 1.00 & 0.00 & $\mu g / L$ \\
\hline 1,2-trans-dichloroethylene & 100 & & 30 & 1.00 & 66.00 & 10.95 & 20.61 & $\mu g / L$ \\
\hline 30-day average & 92 & & 12 & 1.00 & 53.54 & 7.57 & 14.54 & $\mu g / L$ \\
\hline Total zinc & $f$ & & 26 & 0.03 & 68.00 & 17.63 & 15.93 & $\mu g / L$ \\
\hline \multicolumn{9}{|c|}{ Outfall 609 (X-624 carbon filtration facility) } \\
\hline Flow rate & $b$ & & 365 & 0.00 & 0.07 & 0.02 & 0.01 & Mgd \\
\hline Trichloroethylene & 100 & & 27 & 1.00 & 8.00 & 1.96 & 1.63 & $\mu g / L$ \\
\hline 30-day average & 100 & & 12 & 1.00 & 3.66 & 1.85 & 0.85 & $\mu g / L$ \\
\hline
\end{tabular}

National Pollutant Discharge Elimination System.

${ }^{b}$ Flow does not have a numerical limit, no compliance rates are generated.

Insuffiently frequent flow to calculate useful compliance rate.

The permit specifies no detectable polychlorinated biphenyls in the effluent without setting a numerical limit of detection.

No flow throughout entire calendar year.

Monitoring only required.

Table 1.10. Superfund Amendments and Reauthorization Act, Section 313, releases to surface water during 1994 for DOE holdings

\begin{tabular}{clcl}
\hline Chemical name & $\begin{array}{c}\text { Type of environmental } \\
\text { release }\end{array}$ & $\begin{array}{c}\text { Release quantity } \\
(\mathrm{lb})\end{array}$ & Major release sources \\
\hline Zinc & West drainage ditch & 45 & Water treatment \\
& Southwest drainage ditch & 167 & Water treatment \\
\hline
\end{tabular}


2. Environmental Surveillance 
Table 2.1. Quarterly external gamma radiation levels ( $\mu$ rad/hour) for 1994

\begin{tabular}{|c|c|c|c|c|c|c|}
\hline \multirow{2}{*}{ Location } & \multicolumn{4}{|c|}{ Quarter } & \multirow{2}{*}{$\begin{array}{l}\text { Annual dose } \\
\text { ( } \mu \mathrm{rad} / \mathrm{hour})\end{array}$} & \multirow{2}{*}{$\begin{array}{l}\text { Annual dose } \\
\text { (mrem/year) }\end{array}$} \\
\hline & First & Second & Third & Fourth & & \\
\hline \multicolumn{7}{|c|}{ On site } \\
\hline PP518 & 26.2 & 19.9 & 21.2 & 18.0 & 21.2 & \\
\hline PP862 & 21.2 & 26.1 & 23.3 & 24.2 & 23.8 & \\
\hline PP906 & 23.9 & 49.5 & 29.5 & 21.4 & 31.9 & \\
\hline PP933 & 27.1 & $a$ & 22.0 & 17.9 & 22.1 & \\
\hline PP1406 & 20.5 & 16.2 & 21.2 & 18.5 & 19.1 & \\
\hline A35 & 33.9 & 22.2 & 37.8 & 20.6 & 28.9 & \\
\hline $\mathrm{X} 100$ & 26.6 & 17.1 & 19.9 & 12.8 & 16.7 & \\
\hline 611 & 30.0 & $a$ & 35.7 & 26.7 & 31.9 & \\
\hline Mean & 26.04 & 25.16 & 26.33 & 20.26 & 24.6 & 215 \\
\hline $\begin{array}{l}\text { Standard } \\
\text { deviation }\end{array}$ & 4.13 & 11.36 & 6.63 & 4.43 & & \\
\hline $874^{b}$ & 106.4 & 121.7 & 158.6 & 157.2 & 137.5 & 0.60 \\
\hline \multicolumn{7}{|c|}{ Boundary line } \\
\hline A3 & 28.7 & 15.3 & 26.7 & 17.3 & 22.5 & \\
\hline A8 & 24.7 & 23.1 & 25.4 & 24.3 & 24.4 & \\
\hline A9 & 21.3 & 23.8 & 23.5 & 22.0 & 22.8 & \\
\hline A12 & 23.0 & 20.8 & 20.5 & 22.1 & 21.5 & \\
\hline A15 & 43.1 & $a$ & 36.1 & 23.8 & 34.2 & \\
\hline A23 & 54.2 & 16.5 & 26.9 & 20.7 & 26.9 & \\
\hline A24 & 24.7 & 20.4 & 24.9 & 21.2 & 22.8 & \\
\hline A 29 & 23.3 & 21.5 & 22.2 & 19.7 & 21.7 & \\
\hline Mean & 30.4 & 20.5 & 26.0 & 21.4 & 24.4 & 214 \\
\hline $\begin{array}{l}\text { Standard } \\
\text { deviation }\end{array}$ & 11.1 & 2.7 & 4.5 & 2.1 & & \\
\hline \multicolumn{7}{|c|}{ Off site } \\
\hline A6 & 21.8 & 19.9 & 24.6 & 19.4 & 21.6 & 189 \\
\hline A28 & 25.7 & 15.9 & 23.0 & 17.4 & 20.4 & 179 \\
\hline
\end{tabular}

${ }^{\circ}$ Data not acceptable for use.

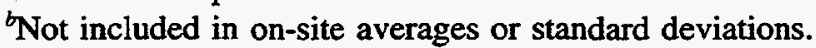


Table 2.2. Airborne gross alpha radiation concentrations $\left(\mathrm{pCl} / \mathrm{m}^{3}\right)$ for 1994

\begin{tabular}{|c|c|c|c|c|c|c|c|c|c|c|c|c|c|c|}
\hline \multirow[b]{2}{*}{ Month } & \multicolumn{14}{|c|}{ Sampling location } \\
\hline & $\begin{array}{c}\mathrm{A} 3 \\
\mathrm{~S}\end{array}$ & $\begin{array}{c}\text { A6 } \\
\text { Pike }^{a}\end{array}$ & $\begin{array}{c}\text { A8 } \\
\text { NW }\end{array}$ & $\begin{array}{l}\text { A9 } \\
\text { SW }\end{array}$ & $\begin{array}{l}\text { A10 } \\
\text { NW }\end{array}$ & $\begin{array}{c}\mathrm{A} 12 \\
\mathrm{E}\end{array}$ & $\begin{array}{l}\text { A15 } \\
\text { SE }\end{array}$ & $\begin{array}{c}\mathrm{A} 23 \\
\mathrm{NE}\end{array}$ & $\begin{array}{c}\mathrm{A} 24 \\
\mathrm{~N}\end{array}$ & $\begin{array}{c}\mathrm{A} 28 \\
\mathrm{Bkg}^{b}\end{array}$ & $\begin{array}{c}\text { A29 } \\
W\end{array}$ & $\begin{array}{c}\text { A36 } \\
X-611\end{array}$ & $\begin{array}{c}\mathrm{A} 37 \\
\mathrm{Bkg}^{b}\end{array}$ & $\begin{array}{c}\mathrm{A} 40 \\
\mathrm{X}-100\end{array}$ \\
\hline January & 0.005 & 0.005 & 0.004 & 0.004 & 0.004 & 0.004 & 0.004 & 0.005 & 0.004 & 0.003 & 0.004 & 0.006 & 0.005 & 0.004 \\
\hline February & $<0.003$ & $<0.004$ & $<0.003$ & $<0.003$ & $<0.003$ & $<0.003$ & $<0.004$ & $<0.003$ & $<0.003$ & $<0.003$ & $<0.003$ & $<0.006$ & 0.008 & $<0.003$ \\
\hline March & $<0.002$ & 0.004 & $<0.002$ & $<0.002$ & $<0.002$ & $<0.002$ & $<0.003$ & $<0.002$ & $<0.002$ & $<0.002$ & 0.002 & $<0.003$ & $<0.002$ & $<0.002$ \\
\hline April & $<0.004$ & $<0.004$ & $<0.003$ & $<0.004$ & $<0.004$ & $<0.004$ & $<0.005$ & $<0.004$ & $<0.003$ & $<0.003$ & $<0.003$ & $<0.005$ & $<0.003$ & $<0.003$ \\
\hline May & $<0.003$ & $<0.004$ & $<0.004$ & $<0.003$ & $<0.003$ & $<0.005$ & $<0.004$ & 0.004 & $<0.003$ & $<0.003$ & $<0.003$ & $<0.004$ & 0.003 & 0.004 \\
\hline June & $<0.003$ & 0.005 & 0.004 & $<0.003$ & $<0.003$ & $<0.003$ & $<0.004$ & $<0.006$ & $<0.003$ & $<0.003$ & $<0.004$ & $<0.005$ & $<0.003$ & $<0.003$ \\
\hline July & $<0.004$ & $<0.005$ & $<0.005$ & $<0.004$ & $<0.006$ & $<0.004$ & $<0.005$ & 0.005 & $<0.004$ & $<0.003$ & $<0.004$ & $<0.005$ & $<0.003$ & $<0.003$ \\
\hline August & $<0.001$ & $<0.001$ & $<0.001$ & $<0.002$ & $<0.003$ & 0.002 & $<0.001$ & 0.004 & 0.003 & 0.003 & 0.003 & $<0.001$ & $<0.001$ & 0.006 \\
\hline September & $<0.004$ & $<0.004$ & $<0.004$ & $<0.004$ & 0.008 & $<0.004$ & $<0.005$ & $<0.004$ & $<0.003$ & 0.003 & $<0.003$ & $<0.005$ & $<0.003$ & $<0.003$ \\
\hline October & 0.006 & $<0.004$ & $<0.004$ & $<0.003$ & $<0.004$ & $<0.004$ & $<0.004$ & $<0.004$ & 0.005 & $<0.003$ & 0.004 & $<0.004$ & $<0.003$ & $<0.003$ \\
\hline November & $<0.003$ & $<0.003$ & $<0.003$ & $<0.003$ & $<0.003$ & $<0.003$ & $<0.003$ & $<0.003$ & $<0.002$ & $<0.003$ & $<0.003$ & $<0.004$ & $<0.002$ & $<0.002$ \\
\hline December & $<0.003$ & 0.004 & $<0.004$ & $<0.003$ & $<0.004$ & $<0.004$ & $<0.004$ & $<0.004$ & $<0.003$ & $<0.003$ & $<0.004$ & $<0.004$ & $<0.003$ & $<0.003$ \\
\hline
\end{tabular}

apiketon.

${ }^{b}$ Background sampling location. 
Table 2.3. Airborne gross beta-gamma radiation concentrations $\left(\mathrm{pC} / \mathrm{m}^{3}\right)$ for 1994

\begin{tabular}{|c|c|c|c|c|c|c|c|c|c|c|c|c|c|c|}
\hline \multirow[b]{2}{*}{ Month } & \multicolumn{14}{|c|}{ Sampling location } \\
\hline & $\begin{array}{c}\text { A3 } \\
\text { S }\end{array}$ & $\begin{array}{c}\text { A6 } \\
\text { Pike }^{a}\end{array}$ & $\begin{array}{l}\text { A8 } \\
\text { NW }\end{array}$ & $\begin{array}{l}\text { A9 } \\
\text { SW }\end{array}$ & $\begin{array}{l}\text { A10 } \\
\text { NW }\end{array}$ & $\mathrm{A} 12$ & $\begin{array}{l}\mathrm{A} 15 \\
\mathrm{SE}\end{array}$ & $\begin{array}{l}\mathrm{A} 23 \\
\mathrm{NE}\end{array}$ & $\begin{array}{c}\mathrm{A} 24 \\
\mathrm{~N}\end{array}$ & $\begin{array}{l}\mathrm{A} 28 \\
\mathbf{B k g}^{b}\end{array}$ & $\begin{array}{c}\text { A29 } \\
\text { W }\end{array}$ & $\begin{array}{c}\mathrm{A} 36 \\
\mathrm{X}-611\end{array}$ & $\begin{array}{l}\mathrm{A} 37 \\
\mathbf{B k g}^{b}\end{array}$ & $\begin{array}{c}\mathrm{A} 40 \\
\mathrm{X}-100\end{array}$ \\
\hline January & 0.034 & 0.045 & 0.036 & 0.037 & 0.022 & 0.024 & 0.022 & 0.045 & 0.023 & $<0.007$ & 0.009 & 0.033 & 0.050 & 0.028 \\
\hline February & 0.021 & 0.032 & 0.026 & 0.026 & 0.025 & 0.020 & 0.029 & 0.026 & 0.018 & 0.024 & 0.025 & 0.029 & 0.059 & 0.023 \\
\hline March & 0.017 & 0.027 & 0.028 & 0.019 & 0.011 & 0.018 & 0.024 & 0.020 & 0.014 & 0.019 & 0.030 & 0.021 & 0.021 & $<0.005$ \\
\hline April & 0.023 & 0.032 & 0.018 & 0.026 & 0.025 & 0.018 & 0.035 & 0.018 & 0.021 & 0.018 & 0.022 & 0.036 & 0.015 & $<0.007$ \\
\hline May & 0.018 & 0.027 & 0.029 & 0.026 & 0.023 & 0.039 & 0.022 & 0.021 & 0.021 & 0.018 & 0.020 & 0.030 & 0.022 & 0.021 \\
\hline June & 0.028 & 0.051 & 0.037 & 0.043 & 0.032 & 0.025 & 0.035 & 0.050 & 0.023 & $<0.006$ & 0.030 & 0.033 & 0.026 & 0.022 \\
\hline July & 0.026 & 0.035 & 0.034 & 0.021 & 0.020 & 0.022 & 0.027 & 0.035 & 0.024 & 0.036 & 0.026 & 0.037 & 0.017 & 0.026 \\
\hline August & 0.024 & 0.033 & 0.022 & 0.023 & $<0.004$ & 0.020 & 0.028 & 0.040 & 0.025 & 0.014 & 0.008 & 0.044 & 0.023 & 0.039 \\
\hline September & 0.053 & 0.047 & 0.009 & 0.039 & 0.060 & 0.034 & 0.039 & 0.041 & 0.030 & 0.036 & 0.017 & 0.039 & 0.031 & 0.031 \\
\hline October & 0.056 & 0.037 & 0.049 & 0.040 & 0.048 & 0.032 & 0.054 & 0.060 & 0.045 & 0.033 & 0.061 & 0.044 & 0.021 & 0.030 \\
\hline November & 0.033 & 0.042 & 0.023 & 0.033 & 0.044 & 0.029 & 0.041 & 0.033 & 0.029 & 0.053 & 0.030 & 0.049 & 0.022 & 0.027 \\
\hline December & 0.040 & 0.057 & 0.030 & 0.044 & 0.052 & 0.036 & 0.050 & 0.057 & 0.037 & 0.039 & 0.039 & 0.061 & 0.027 & 0.031 \\
\hline \multicolumn{15}{|c|}{$\begin{array}{l}\text { Piketon. } \\
{ }^{b} \text { Background samping location. }\end{array}$} \\
\hline
\end{tabular}


Table 2.4. Airborne gross gaseous fluoride concentrations $\left(\mu \mathrm{g} / \mathrm{m}^{3}\right)$ for 1994

\begin{tabular}{|c|c|c|c|c|c|c|c|c|c|c|c|c|c|c|}
\hline \multirow[b]{2}{*}{ Week } & \multicolumn{3}{|c|}{ On-site air sampling stations } & \multicolumn{8}{|c|}{ Off-site (fence-line) air sampling stations } & \multicolumn{3}{|c|}{ Off-site air sampling stations } \\
\hline & $\begin{array}{c}\mathrm{A} 10 \\
\mathrm{X}-230-\mathrm{J} 3\end{array}$ & $\begin{array}{c}\text { A36 } \\
\text { X-611 }\end{array}$ & $\begin{array}{c}\text { A40 } \\
\text { X-100 }\end{array}$ & $\begin{array}{c}\text { A3 } \\
\text { S }\end{array}$ & $\begin{array}{c}\text { A8 } \\
\text { NW }\end{array}$ & $\begin{array}{l}\text { A9 } \\
\text { SW }\end{array}$ & $\begin{array}{c}\mathrm{A} 12 \\
\mathrm{E}\end{array}$ & $\begin{array}{c}\text { A15 } \\
\text { SE }\end{array}$ & $\begin{array}{l}\text { A23 } \\
\mathrm{NE}\end{array}$ & $\begin{array}{c}\mathrm{A} 24 \\
\mathrm{~N}\end{array}$ & $\begin{array}{c}\text { A29 } \\
\text { W }\end{array}$ & $\begin{array}{c}\text { A6 } \\
\text { Pike }^{a}\end{array}$ & $\begin{array}{c}\mathrm{A} 28 \\
\mathrm{Bkg}^{b}\end{array}$ & $\begin{array}{c}\mathrm{A} 37 \\
\mathrm{Bkg}^{b}\end{array}$ \\
\hline \multicolumn{15}{|l|}{ January } \\
\hline 11 & 0.06 & 0.09 & 0.47 & $<0.07$ & $<0.07$ & $<0.06$ & 0.09 & 0.13 & $<0.09$ & 0.08 & $<0.07$ & $<0.08$ & $<0.07$ & $<0.07$ \\
\hline 25 & 0.10 & 0.13 & 0.46 & 0.07 & 0.06 & 0.05 & 0.10 & 0.17 & 0.04 & 0.08 & 0.06 & 0.05 & 0.04 & 0.06 \\
\hline \multicolumn{15}{|l|}{ February } \\
\hline 1 & 0.11 & 0.13 & 0.38 & 0.10 & 0.08 & 0.11 & 0.10 & 0.13 & 0.08 & 0.08 & 0.09 & $<0.09$ & 0.07 & 0.07 \\
\hline 8 & $<0.06$ & 0.16 & 0.16 & $<0.06$ & 0.06 & $<0.06$ & $<0.06$ & $<0.08$ & $<0.06$ & $<0.05$ & $<0.06$ & $<0.08$ & $<0.05$ & $<0.05$ \\
\hline 14 & 0.07 & 0.18 & $<0.07$ & 0.10 & 0.10 & 0.10 & 0.14 & 0.13 & $<0.08$ & 0.07 & 0.10 & 0.10 & 0.12 & $<0.10$ \\
\hline 22 & 0.05 & 0.13 & 0.11 & $<0.06$ & 0.07 & $<0.06$ & 0.08 & $<0.07$ & 0.08 & $<0.05$ & $<0.06$ & $<0.09$ & $<0.06$ & $<0.07$ \\
\hline \multicolumn{15}{|l|}{ March } \\
\hline 1 & $<0.06$ & $<0.11$ & $<0.06$ & $<0.09$ & $<0.06$ & $<0.07$ & 0.06 & $<0.08$ & $<0.07$ & $<0.06$ & $<0.05$ & $<0.07$ & $<0.05$ & $<0.05$ \\
\hline 7 & 0.07 & $<0.15$ & 0.21 & $<0.07$ & $<0.08$ & $<0.08$ & 0.08 & $<0.10$ & $<0.08$ & $<0.07$ & 0.08 & $<0.10$ & 0.08 & $<0.07$ \\
\hline 14 & $<0.06$ & $<0.11$ & $<0.05$ & $<0.06$ & $<0.06$ & $<0.06$ & 0.11 & $<0.08$ & $<0.07$ & $<0.06$ & 0.08 & $<0.09$ & $<0.06$ & $<0.05$ \\
\hline 21 & 0.08 & $<0.11$ & $<0.06$ & $<0.06$ & $<0.06$ & ND & 0.07 & 0.10 & 0.07 & 0.06 & 0.10 & $<0.09$ & $<0.06$ & 0.07 \\
\hline 28 & 0.07 & 0.15 & 0.08 & $<0.06$ & 0.07 & $<0.07$ & 0.10 & $<0.08$ & 0.07 & 0.06 & 0.09 & $<0.09$ & $<0.06$ & $<0.05$ \\
\hline \multicolumn{15}{|l|}{ April } \\
\hline 5 & 0.07 & 0.16 & 0.05 & $<0.05$ & $<0.05$ & $<0.05$ & $<0.05$ & $<0.07$ & $<0.06$ & 0.06 & 0.07 & $<0.08$ & $<0.07$ & $<0.06$ \\
\hline 11 & $<0.09$ & $<0.14$ & 0.06 & $<0.09$ & $<0.07$ & $<0.10$ & 0.07 & $<0.09$ & 0.08 & $<0.07$ & 0.09 & $<0.11$ & $<0.06$ & $<0.05$ \\
\hline 18 & 0.12 & 0.60 & 0.36 & $<0.10$ & 0.08 & 0.08 & 0.09 & $<0.12$ & $<0.09$ & $<0.07$ & $<0.09$ & $<0.09$ & $<0.08$ & $<0.05$ \\
\hline 25 & $<0.07$ & 0.14 & 0.37 & $<0.07$ & 0.06 & $<0.07$ & $<0.06$ & $<0.09$ & $<0.08$ & 0.06 & $<0.08$ & $<0.10$ & $<0.08$ & $<0.05$ \\
\hline \multicolumn{15}{|l|}{ May } \\
\hline 2 & 0.10 & 0.11 & 0.15 & 0.13 & 0.06 & 0.09 & 0.09 & $<0.11$ & 0.07 & 0.08 & $<0.09$ & $<0.06$ & $<0.09$ & $<0.05$ \\
\hline 9 & 0.09 & 0.18 & 0.30 & 0.09 & $<0.06$ & 0.08 & 0.12 & $<0.13$ & 0.09 & $<0.08$ & $<0.06$ & $<0.11$ & 0.11 & 0.05 \\
\hline 16 & 0.09 & 0.13 & 0.25 & $<0.07$ & $<0.06$ & $<0.07$ & 0.06 & 0.10 & 0.11 & $<0.07$ & $<0.08$ & $<0.06$ & $<0.06$ & $<0.06$ \\
\hline
\end{tabular}




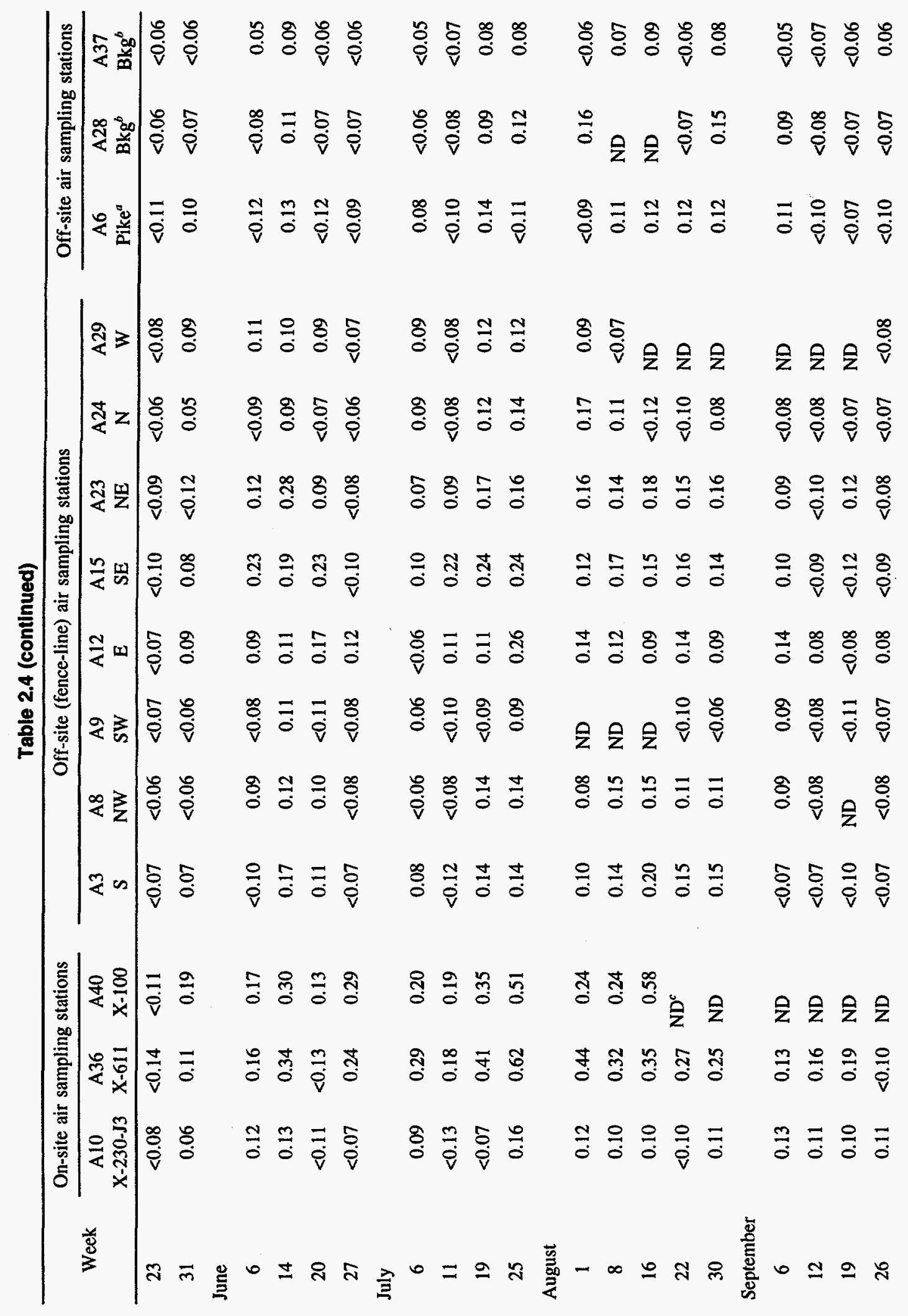


Table 2.4 (continued)

\begin{tabular}{|c|c|c|c|c|c|c|c|c|c|c|c|c|c|c|}
\hline \multirow[b]{2}{*}{ Week } & \multicolumn{3}{|c|}{ On-site air sampling stations } & \multicolumn{8}{|c|}{ Off-site (fence-line) air sampling stations } & \multicolumn{3}{|c|}{ Off-site air sampling stations } \\
\hline & $\begin{array}{c}\mathrm{A} 10 \\
\mathrm{X}-230-\mathrm{J} 3\end{array}$ & $\begin{array}{c}\text { A36 } \\
X-611 \\
\end{array}$ & $\begin{array}{c}\mathrm{A} 40 \\
\mathrm{X}-100 \\
\end{array}$ & $\begin{array}{c}\mathrm{A} 3 \\
\mathrm{~S}\end{array}$ & $\begin{array}{c}\text { A8 } \\
\text { NW }\end{array}$ & $\begin{array}{l}\text { A9 } \\
\text { SW } \\
\end{array}$ & $\begin{array}{c}\mathrm{A} 12 \\
\mathrm{E}\end{array}$ & $\begin{array}{c}\text { A15 } \\
\text { SE }\end{array}$ & $\begin{array}{l}\mathrm{A} 23 \\
\mathrm{NE}\end{array}$ & $\begin{array}{c}\mathrm{A} 24 \\
\mathrm{~N}\end{array}$ & $\begin{array}{c}\text { A29 } \\
\text { W }\end{array}$ & $\begin{array}{c}\text { A6 } \\
\text { Pike }^{a}\end{array}$ & $\begin{array}{c}\mathbf{A} 28 \\
\mathbf{B k g}^{b} \\
\end{array}$ & $\begin{array}{r}\text { A37 } \\
\text { Bkg }^{b} \\
\end{array}$ \\
\hline \multicolumn{15}{|l|}{ October } \\
\hline 3 & 0.08 & 0.07 & ND & $<0.07$ & $<0.07$ & $<0.06$ & $<0.08$ & $<0.09$ & $<0.08$ & $<0.06$ & $<0.06$ & $<0.07$ & $<0.07$ & $<0.06$ \\
\hline 11 & $<0.06$ & $<0.11$ & 0.06 & $<0.05$ & $<0.07$ & $<0.06$ & $<0.07$ & $<0.06$ & $<0.09$ & $<0.06$ & $<0.06$ & $<0.08$ & $<0.06$ & $<0.05$ \\
\hline 17 & $<0.11$ & $<0.13$ & 0.09 & $<0.07$ & $<0.07$ & $<0.08$ & $<0.08$ & $<0.10$ & $<0.11$ & $<0.09$ & 0.08 & $<0.08$ & $<0.08$ & $<0.06$ \\
\hline 24 & $<0.06$ & 0.13 & 0.14 & $<0.06$ & $<0.06$ & $<0.04$ & 0.10 & $<0.06$ & $<0.09$ & $<0.05$ & $<0.06$ & $<0.07$ & $<0.08$ & $<0.08$ \\
\hline \multicolumn{15}{|l|}{ November } \\
\hline 1 & $<0.09$ & 0.14 & 0.14 & $<0.06$ & $<0.05$ & $<0.05$ & $<0.08$ & $<0.07$ & $<0.08$ & $<0.05$ & $<0.06$ & $<0.08$ & $<0.06$ & $<0.06$ \\
\hline 8 & $<0.10$ & 0.14 & 0.11 & $<0.07$ & $<0.07$ & $<0.06$ & 0.11 & $<0.08$ & 0.09 & 0.06 & $<0.06$ & $<0.07$ & $<0.06$ & $<0.06$ \\
\hline 14 & $<0.11$ & 0.11 & $<0.08$ & $<0.07$ & $<0.06$ & $<0.06$ & $<0.09$ & $<0.08$ & $<0.09$ & $<0.09$ & $<0.06$ & $<0.09$ & $<0.08$ & $<0.08$ \\
\hline 21 & $<0.09$ & 0.09 & 0.07 & 0.07 & $<0.06$ & $<0.05$ & 0.10 & $<0.08$ & $<0.09$ & $<0.06$ & $<0.06$ & $<0.07$ & $<0.07$ & $<0.07$ \\
\hline 28 & $<0.08$ & 0.12 & 0.47 & $<0.06$ & $<0.06$ & $<0.05$ & 0.10 & $<0.07$ & $<0.08$ & $<0.06$ & $<0.07$ & $<0.09$ & $<0.07$ & $<0.06$ \\
\hline \multicolumn{15}{|l|}{ December } \\
\hline 5 & $<0.08$ & 0.14 & 0.29 & $<0.06$ & $<0.07$ & $<0.05$ & 0.12 & $<0.08$ & $<0.10$ & 0.06 & $<0.06$ & $<0.06$ & $<0.09$ & $<0.06$ \\
\hline 12 & $<0.08$ & $<0.11$ & 0.08 & $<0.06$ & $<0.05$ & $<0.05$ & 0.08 & $<0.07$ & $<0.07$ & $<0.08$ & $<0.06$ & $<0.08$ & $<0.06$ & $<0.06$ \\
\hline 19 & $<0.08$ & $<0.08$ & 0.12 & $<0.06$ & $<0.06$ & 0.05 & $<0.08$ & $<0.07$ & $<0.08$ & $<0.06$ & $<0.07$ & $<0.08$ & $<0.07$ & 0.07 \\
\hline 27 & $<0.07$ & $<0.06$ & $<0.05$ & $<0.05$ & $<0.06$ & $<0.05$ & $<0.07$ & $<0.07$ & $<0.07$ & $<0.05$ & $<0.06$ & $<0.07$ & $<0.06$ & $<0.06$ \\
\hline \multicolumn{15}{|c|}{ January '95 } \\
\hline 3 & $<0.07$ & $<0.08$ & $<0.06$ & $<0.05$ & $<0.08$ & 0.06 & $<0.10$ & $<0.07$ & $<0.10$ & $<0.05$ & $<0.08$ & $<0.06$ & $<0.08$ & $<0.07$ \\
\hline
\end{tabular}

"Piketon.

${ }^{b}$ Background sampling location.

No data available. 
3. Dose 


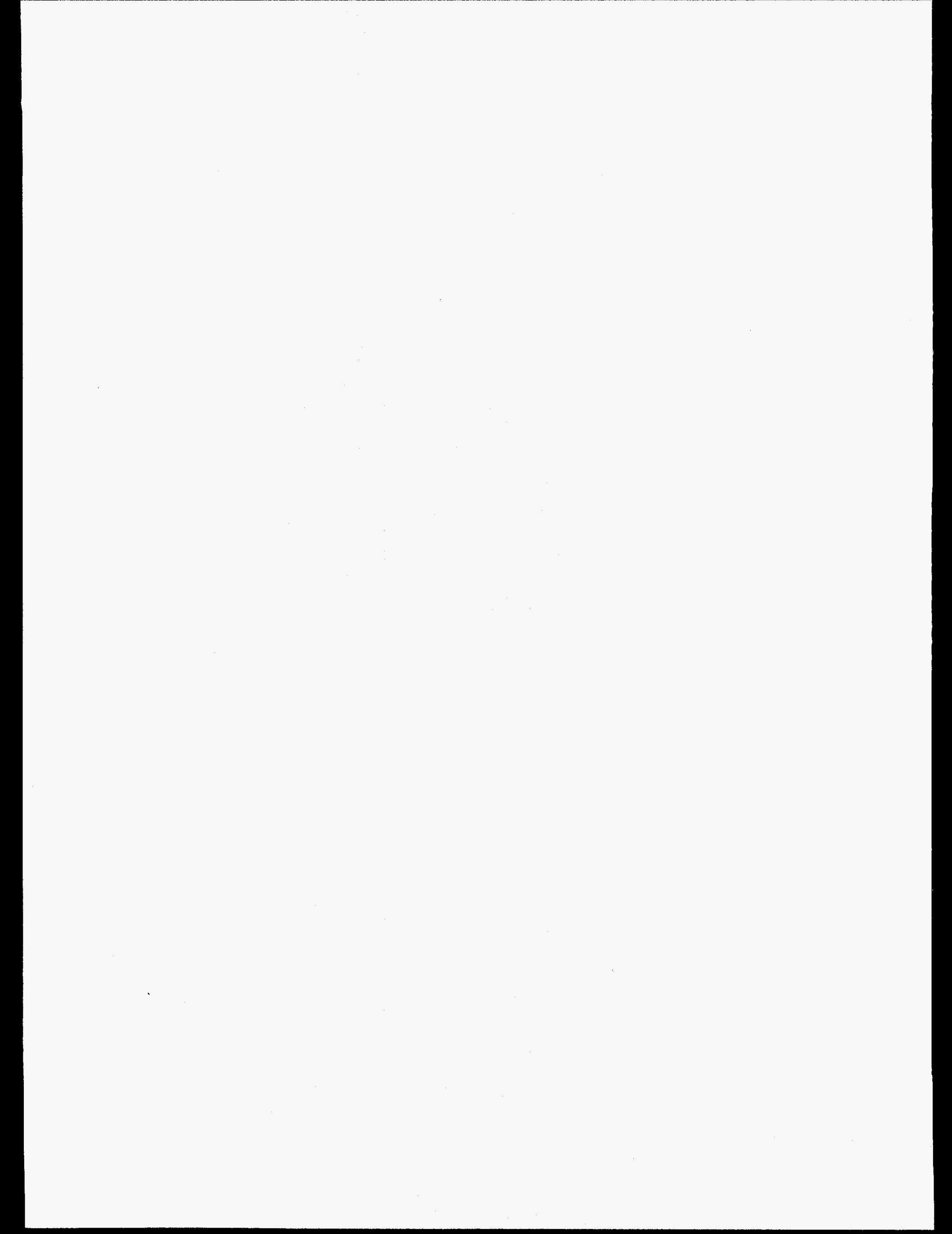


Table 3.1. Committed effective dose rate conversion factors ${ }^{a}$

\begin{tabular}{lcccc}
\hline $\begin{array}{c}\text { Radionuclide } \\
\text { (solubility) }\end{array}$ & $\begin{array}{c}\text { Inhalation } \\
(\mathrm{rem} / \mu \mathrm{Ci})\end{array}$ & $\begin{array}{c}\text { Ingestion } \\
(\mathrm{rem} / \mu \mathrm{Ci})\end{array}$ & $\begin{array}{c}\text { Immersion } \\
(\mathrm{mrem} / \text { year } \\
\left.\text { per } \mu \mathrm{Ci} / \mathrm{cm}^{3}\right)\end{array}$ & $\begin{array}{c}\text { Ground surfaces } \\
(\mathrm{mrem} / \mathrm{year} \\
\left.\text { per } \mu \mathrm{Ci} / \mathrm{cm}^{2}\right)\end{array}$ \\
\hline${ }^{99} \mathrm{Tc}(\mathrm{D}){ }^{b}$ & $1.03 \times 10^{-3}$ & $1.45 \times 10^{-3}$ & $2.55 \times 10^{3}$ & $6.03 \times 10^{-1}$ \\
${ }^{234} \mathrm{U}(\mathrm{D})$ & 2.67 & $2.74 \times 10^{-1}$ & $7.46 \times 10^{5}$ & $8.00 \times 10^{2}$ \\
${ }^{235} \mathrm{U}(\mathrm{D})$ & 2.54 & $2.64 \times 10^{-1}$ & $7.51 \times 10^{8}$ & $1.67 \times 10^{5}$ \\
${ }^{236} \mathrm{U}(\mathrm{D})$ & 2.53 & $2.60 \times 10^{-1}$ & $5.90 \times 10^{5}$ & $7.27 \times 10^{2}$ \\
${ }^{238} \mathrm{U}(\mathrm{D})$ & 2.40 & $2.47 \times 10^{-1}$ & $5.06 \times 10^{5}$ & $6.41 \times 10^{2}$ \\
${ }^{231} \mathrm{Th}(\mathrm{W})$ & $9.62 \times 10^{b}$ & $1.35 \times 10^{-3}$ & $5.82 \times 10^{1}$ & 1.91 \\
${ }^{234} \mathrm{Th}(\mathrm{W})$ & $3.06 \times 10^{-2}$ & $1.37 \times 10^{-2}$ & $3.73 \times 10^{7}$ & $9.71 \times 10^{3}$ \\
${ }^{234 m} \mathrm{~Pa}(\mathrm{~W})$ & $3.77 \times 10^{-6}$ & $5.90 \times 10^{-6}$ & $5.93 \times 10^{7}$ & $1.12 \times 10^{4}$ \\
\hline
\end{tabular}

${ }^{a}$ Factors taken from the ALLRAD88 data file provided with CAP-88.

${ }^{b} \mathrm{D}=$ soluble in blood; $\mathrm{W}=$ moderately soluble in blood.

Table 3.2. Release point parameters and receptor locations used in dose calculations

\begin{tabular}{lcccccc}
\hline Name & Type & $\begin{array}{c}\text { Release } \\
\text { height } \\
(\mathrm{m})\end{array}$ & $\begin{array}{c}\text { Inner } \\
\text { diameter } \\
(\mathrm{m})\end{array}$ & $\begin{array}{c}\text { Gas exit } \\
\text { velocity } \\
(\mathrm{m} / \mathrm{s})\end{array}$ & $\begin{array}{c}\text { Gas exit } \\
\text { temperature } \\
\left({ }^{\circ} \mathrm{C}\right)\end{array}$ & $\begin{array}{c}\text { Distance }(\mathrm{m}) \text { and } \\
\text { direction to } \\
\text { receptor }^{a}\end{array}$ \\
\hline Stack 1 & Point & 50 & 0.25 & 0 & Ambient & $1770 \mathrm{ENE}$ \\
Stack 2 & Point & 20 & 0.57 & 0 & Ambient & $1770 \mathrm{ENE}$ \\
Stack $3^{b}$ & Point & 3 & 0.10 & 0 & Ambient & $1770 \mathrm{ENE}$ \\
\hline
\end{tabular}

${ }^{a}$ Receptor is residence of maximally exposed member of public.

bDOE source. 
Table 3.3. Activities (Ci), solubilities, and AMAD ${ }^{\alpha}(\mu \mathrm{m})$ of radionuclides released from Portsmouth during 1994

\begin{tabular}{lcccc}
\hline \multirow{2}{*}{$\begin{array}{c}\text { Radionuclide } \\
\text { (solubility) }\end{array}$} & $\begin{array}{c}\text { AMAD } \\
(\mu \mathrm{m})\end{array}$ & $\begin{array}{c}\text { Stack 1 } \\
\text { USEC (1) }\end{array}$ & $\begin{array}{c}\text { Stack 2 } \\
\text { USEC (2) }\end{array}$ & $\begin{array}{c}\text { Stack 3c } \\
\text { DOE }\end{array}$ \\
\cline { 3 - 5 } & 1.0 & $1.219 \mathrm{E}-1$ & $1.581 \mathrm{E}-2$ & $1.115 \mathrm{E}-3$ \\
\hline${ }^{99} \mathrm{Tc}(\mathrm{D}){ }^{b}$ & 1.0 & $3.220 \mathrm{E}-2$ & $4.243 \mathrm{E}-3$ & $8.755 \mathrm{E}-5$ \\
${ }^{234} \mathrm{U}(\mathrm{D})$ & 1.0 & $7.693 \mathrm{E}-4$ & $1.972 \mathrm{E}-4$ & $2.413 \mathrm{E}-6$ \\
${ }^{235} \mathrm{U}(\mathrm{D})$ & 1.0 & $7.470 \mathrm{E}-6$ & $3.864 \mathrm{E}-6$ & $7.578 \mathrm{E}-9$ \\
${ }^{236} \mathrm{U}(\mathrm{D})$ & 1.0 & $4.634 \mathrm{E}-4$ & $2.016 \mathrm{E}-3$ & $3.863 \mathrm{E}-6$ \\
${ }^{238} \mathrm{U}(\mathrm{D})$ & 1.0 & $7.693 \mathrm{E}-4$ & $1.972 \mathrm{E}-4$ & $5.777 \mathrm{E}-6$ \\
${ }^{231} \mathrm{Th}(\mathrm{W})$ & 1.0 & $4.643 \mathrm{E}-4$ & $2.016 \mathrm{E}-3$ & $3.655 \mathrm{E}-6$ \\
${ }^{234} \mathrm{Th}(\mathrm{W})$ & 1.0 & $4.643 \mathrm{E}-4$ & $2.016 \mathrm{E}-3$ & $3.395 \mathrm{E}-6$ \\
${ }^{234 m} \mathrm{~Pa}(\mathrm{~W})$ & &
\end{tabular}

${ }^{a}$ Activity median aerodynamic diameter.

${ }^{b} \mathrm{D}=$ soluble in blood; $\mathrm{W}=$ moderately soluble in blood.

Represents $27 \%$ of curies released.

Table 3.4. Predicted radiation doses from airborne releases at the Portsmouth site during 1994

Effective dose equivalent (EDE) to:

EDE

\begin{tabular}{ll}
\hline Maximum exposed individual $^{a}$ & 0.06 mrem \\
Population $^{b}$ & 0.6 person-rem \\
Nearest community $^{c}$ & 0.02 person-rem \\
\hline
\end{tabular}

"Maximum exposed individual resides on the reservation boundary, $1770 \mathrm{~m}(5807 \mathrm{ft}$ ) ENE of plant center.

${ }^{b}$ Collective EDE to general population with $80 \mathrm{~km}$ (50 miles) of plant site.

${ }^{c}$ Collective EDE to residents of nearest community (Piketon, Ohio). 
Table 3.5. Predicted radiation doses from waterborne releases at the Portsmouth site during 1994

Pathway of exposure ${ }^{a}$

Effective dose equivalent

Drinking water from Scioto River ${ }^{b}$

0.0052

Eating fish from Scioto River ${ }^{c}$

0.0015

Swimming in Scioto River

$<0.0001$

Boating on Scioto River

$<0.0001$

Shoreline use ${ }^{f}$

$<0.0001$

Total

0.007

${ }^{\circ}$ Point of maximum exposure is the Scioto River downstream of the Portsmouth site.

${ }^{b}$ Assumed consumption of $730 \mathrm{~L}$ (193 gal) of water per year.

${ }^{c}$ Assumed consumption of $21 \mathrm{~kg}(46 \mathrm{lb})$ of fish per year.

${ }^{d}$ Assumed total time of 27 hours per year.

eAssumed total time of 105 hours per year.

${ }^{f}$ Assumed total time of 69 hours per year. 

Table 4.1. Portsmouth groundwater monitoring for 1994: trichloroethylene results for the X-701B holding pond

\begin{tabular}{|c|c|c|c|c|c|c|}
\hline Well & Parameter & $\operatorname{Min}^{a}$ & $\operatorname{Max}^{a}$ & Mean $^{b}$ & $\begin{array}{c}\text { Standard } \\
\text { deviation }^{b}\end{array}$ & Units \\
\hline \multicolumn{7}{|c|}{ Gallia wells } \\
\hline $\mathrm{X} 701-02 \mathrm{G}$ & Trichloroethylene & 4 & 5 & 3.8 & 1.3 & $\mu \mathrm{g} / \mathrm{L}$ \\
\hline $\mathrm{X701-05G}$ & Trichloroethylene & 7 & 10 & 9.0 & 1.4 & $\mu \mathrm{g} / \mathrm{L}$ \\
\hline $\mathrm{X701-06G}$ & Trichloroethylene & 79 & 225 & 148 & 60.6 & $\mu \mathrm{g} / \mathrm{L}$ \\
\hline X701-08G & Trichloroethylene & 153,000 & 449,000 & 260,250 & 133,829 & $\mu \mathrm{g} / \mathrm{L}$ \\
\hline $\mathrm{X} 701-10 \mathrm{G}$ & Trichloroethylene & 1400 & 1770 & 1620 & 159 & $\mu \mathrm{g} / \mathrm{L}$ \\
\hline $\mathrm{X} 701-14 \mathrm{G}$ & Trichloroethylene & 163,000 & 430,000 & 341,250 & 124,973 & $\mu \mathrm{g} / \mathrm{L}$ \\
\hline$X 701-15 G$ & Trichloroethylene & 6 & 17 & 12.0 & 5.0 & $\mu \mathrm{g} / \mathrm{L}$ \\
\hline $\mathrm{X} 701-16 \mathrm{G}$ & Trichloroethylene & $2 U$ & $2 U$ & NA & NA & $\mu \mathrm{g} / \mathrm{L}$ \\
\hline $\mathrm{X} 701-19 \mathrm{G}$ & Trichloroethylene & $2 U$ & $2 U$ & NA & $\mathrm{NA}$ & $\mu \mathrm{g} / \mathrm{L}$ \\
\hline$X 701-21 G$ & Trichloroethylene & 77 & 94 & 82.8 & 7.6 & $\mu \mathrm{g} / \mathrm{L}$ \\
\hline$X 701-23 G$ & Trichloroethylene & $2 \mathrm{U}$ & $2 \mathrm{U}$ & NA & NA & $\mu \mathrm{g} / \mathrm{L}$ \\
\hline$X 701-24 G$ & Trichloroethylene & 41,900 & 92,900 & 64,475 & 21,098 & $\mu \mathrm{g} / \mathrm{L}$ \\
\hline $\mathrm{X} 701-25 \mathrm{G}$ & Trichloroethylene & $2 U$ & $2 \mathrm{U}$ & NA & NA & $\mu \mathrm{g} / \mathrm{L}$ \\
\hline$X 701-30 G$ & Trichloroethylene & 17 & 22 & 19.0 & 2.2 & $\mu \mathrm{g} / \mathrm{L}$ \\
\hline$X 701-31 G$ & Trichloroethylene & $2 \mathrm{U}$ & $2 U$ & NA & NA & $\mu \mathrm{g} / \mathrm{L}$ \\
\hline $\mathrm{X} 701-32 \mathrm{G}$ & Trichloroethylene & $2 \mathrm{U}$ & $2 \mathrm{U}$ & NA & NA & $\mu \mathrm{g} / \mathrm{L}$ \\
\hline $\mathrm{X701-38G}$ & Trichloroethylene & $2 \mathrm{U}$ & $2 U$ & $\mathrm{NA}$ & NA & $\mu \mathrm{g} / \mathrm{L}$ \\
\hline$X 701-48 G$ & Trichloroethylene & $2 \mathrm{U}$ & $2 U$ & $\mathrm{NA}$ & NA & $\mu \mathrm{g} / \mathrm{L}$ \\
\hline $\mathrm{X701-49G}$ & Trichloroethylene & $2 \mathrm{U}$ & $2 \mathrm{U}$ & $\mathrm{NA}$ & NA & $\mu \mathrm{g} / \mathrm{L}$ \\
\hline X701-BW1G & Trichloroethylene & $2 \mathrm{U}$ & $2 \mathrm{U}$ & NA & NA & $\mu \mathrm{g} / \mathrm{L}$ \\
\hline X701-BW4G & Trichloroethylene & $2 \mathrm{U}$ & $2 \mathrm{U}$ & NA & NA & $\mu \mathrm{g} / \mathrm{L}$ \\
\hline LBC-PZ03 & Trichloroethylene & 367 & 1070 & 727 & 337 & $\mu \mathrm{g} / \mathrm{L}$ \\
\hline LBC-PZ06 & Trichloroethylene & $2 \mathrm{U}$ & $2 \mathrm{U}$ & NA & NA & $\mu \mathrm{g} / \mathrm{L}$ \\
\hline \multicolumn{7}{|c|}{ Berea wells } \\
\hline $\mathrm{X} 701-50 \mathrm{~B}$ & Trichloroethylene & $2 \mathrm{U}$ & $2 \mathrm{U}$ & NA & NA & $\mu \mathrm{g} / \mathrm{L}$ \\
\hline $\mathrm{X} 701-58 \mathrm{~B}$ & Trichloroethylene & $2 U$ & $2 U$ & NA & NA & $\mu \mathrm{g} / \mathrm{L}$ \\
\hline $\mathrm{X} 701-61 \mathrm{~B}$ & Trichloroethylene & $2 U$ & $2 U$ & NA & NA & $\mu \mathrm{g} / \mathrm{L}$ \\
\hline
\end{tabular}

${ }^{a}$ Qualifiers include: U-parameter was not detected; the result is the attainable detection limit for the sample.

"NA-at least $50 \%$ of the results for the given parameter at this location were below detection; therefore, mean and standard deviation functions are not applicable. 
Table 4.2. Portsmouth groundwater monitoring for 1994: radionuclide results for the $\mathrm{X}-701 \mathrm{~B}$ holding pond

\begin{tabular}{|c|c|c|c|c|c|c|}
\hline Well & Parameter & $\operatorname{Min}^{a}$ & $\operatorname{Max}^{a}$ & Mean $^{b}$ & $\begin{array}{c}\text { Standard } \\
\text { deviation }^{b}\end{array}$ & Units \\
\hline \multicolumn{7}{|c|}{ Gallia wells } \\
\hline \multirow[t]{4}{*}{$X 701-02 G$} & Gross alpha & $5 \mathrm{U}$ & $5 \mathrm{U}$ & NA & NA & $\mathrm{pCi} / \mathrm{L}$ \\
\hline & Gross beta & $9 \mathrm{U}$ & 11 & NA & NA & $\mathrm{pCi} / \mathrm{L}$ \\
\hline & Technetium & $22 \mathrm{U}$ & 30 & NA & NA & $\mathrm{pCi} / \mathrm{L}$ \\
\hline & Total uranium & $1.0 \mathrm{U}$ & $1.0 \mathrm{U}$ & NA & NA & $\mu \mathrm{g} / \mathrm{L}$ \\
\hline \multirow[t]{4}{*}{$X 701-05 G$} & Gross alpha & $5 \mathrm{U}$ & $6 \mathrm{U}$ & NA & NA & $\mathrm{pCi} / \mathrm{L}$ \\
\hline & Gross beta & $9 \mathrm{U}$ & $11 \mathrm{U}$ & NA & NA & $\mathrm{pCi} / \mathrm{L}$ \\
\hline & Technetium & $22 \mathrm{U}$ & $22 \mathrm{U}$ & NA & NA & $\mathrm{pCi} / \mathrm{L}$ \\
\hline & Total uranium & $1.0 \mathrm{U}$ & $1.0 \mathrm{U}$ & NA & NA & $\mu \mathrm{g} / \mathrm{L}$ \\
\hline \multirow[t]{4}{*}{ X701-06G } & Gross alpha & $2 \mathrm{U}$ & $7 \mathrm{U}$ & NA & $\mathrm{NA}$ & $\mathrm{pCi} / \mathrm{L}$ \\
\hline & Gross beta & 12 & 30 & 22.0 & 7.5 & $\mathrm{pCi} / \mathrm{L}$ \\
\hline & Technetium & $24 \mathrm{U}$ & 73 & 42.3 & 26.0 & $\mathrm{pCi} / \mathrm{L}$ \\
\hline & Total uranium & $1.0 \mathrm{U}$ & $1.0 \mathrm{U}$ & NA & NA & $\mu \mathrm{g} / \mathrm{L}$ \\
\hline \multirow[t]{4}{*}{$X 701-08 G$} & Gross alpha & $6 \mathrm{U}$ & 7 & NA & NA & $\mathrm{pCi} / \mathrm{L}$ \\
\hline & Gross beta & 77 & 193 & 122.5 & 52.9 & $\mathrm{pCi} / \mathrm{L}$ \\
\hline & Technetium & 118 & 238 & 173.0 & 52.2 & $\mathrm{pCi} / \mathrm{L}$ \\
\hline & Total uranium & $1.0 \mathrm{U}$ & $1.0 \mathrm{U}$ & $\mathrm{NA}$ & NA & $\mu \mathrm{g} / \mathrm{L}$ \\
\hline \multirow[t]{4}{*}{$X 701-10 G$} & Gross alpha & $6 \mathrm{U}$ & $7 \mathrm{U}$ & NA & NA & $\mathrm{pCi} / \mathrm{L}$ \\
\hline & Gross beta & $9 \mathrm{U}$ & $10 \mathrm{U}$ & NA & NA & $\mathrm{pCi} / \mathrm{L}$ \\
\hline & Technetium & $22 \mathrm{U}$ & $22 \mathrm{U}$ & NA & NA & $\mathrm{pCi} / \mathrm{L}$ \\
\hline & Total uranium & $1.0 \mathrm{U}$ & $1.0 \mathrm{U}$ & NA & NA & $\mu \mathrm{g} / \mathrm{L}$ \\
\hline \multirow[t]{4}{*}{$X 701-14 G$} & Gross alpha & $6 \mathrm{U}$ & $7 \mathrm{U}$ & NA & NA & $\mathrm{pCi} / \mathrm{L}$ \\
\hline & Gross beta & 20 & 131 & 77.8 & 55.4 & $\mathrm{pCi} / \mathrm{L}$ \\
\hline & Technetium & 34 & 162 & 91.3 & 61.9 & $\mathrm{pCi} / \mathrm{L}$ \\
\hline & Total uranium & $1.0 \mathrm{U}$ & $1.0 \mathrm{U}$ & NA & NA & $\mu \mathrm{g} / \mathrm{L}$ \\
\hline \multirow[t]{4}{*}{ X701-15G } & Gross alpha & $3 \mathrm{U}$ & 9 & NA & NA & $\mathrm{pCi} / \mathrm{L}$ \\
\hline & Gross beta & $9 \mathrm{U}$ & $19 \mathrm{U}$ & NA & NA & $\mathrm{pCi} / \mathrm{L}$ \\
\hline & Technetium & $22 \mathrm{U}$ & $24 U$ & NA & NA & $\mathrm{pCi} / \mathrm{L}$ \\
\hline & Total uranium & $1.0 \mathrm{U}$ & $1.0 \mathrm{U}$ & NA & NA & $\mu \mathrm{g} / \mathrm{L}$ \\
\hline \multirow[t]{4}{*}{$\mathrm{X701-16G}$} & Gross alpha & $4 \mathrm{U}$ & 9 & NA & NA & $\mathrm{pCi} / \mathrm{L}$ \\
\hline & Gross beta & $9 \mathrm{U}$ & 15 & $\mathrm{NA}$ & NA & $\mathrm{pCi} / \mathrm{L}$ \\
\hline & Technetium & $22 \mathrm{U}$ & 25 & $\mathrm{NA}$ & NA & $\mathrm{pCi} / \mathrm{L}$ \\
\hline & Total uranium & $1.0 \mathrm{U}$ & 1.3 & 1.0 & 0.3 & $\mu \mathrm{g} / \mathrm{L}$ \\
\hline \multirow[t]{4}{*}{$\mathrm{X} 701-19 \mathrm{G}$} & Gross alpha & $4 U$ & $4 \mathrm{U}$ & NA & NA & $\mathrm{pCi} / \mathrm{L}$ \\
\hline & Gross beta & $9 \mathrm{U}$ & $11 \mathrm{U}$ & NA & NA & $\mathrm{pCi} / \mathrm{L}$ \\
\hline & Technetium & $22 \mathrm{U}$ & $24 \mathrm{U}$ & NA & NA & $\mathrm{pCi} / \mathrm{L}$ \\
\hline & Total uranium & $1.0 \mathrm{U}$ & $1.0 \mathrm{U}$ & NA & NA & $\mu \mathrm{g} / \mathrm{L}$ \\
\hline
\end{tabular}


Table 4.2 (continued)

\begin{tabular}{|c|c|c|c|c|c|c|}
\hline Well & Parameter & $\operatorname{Min}^{a}$ & $\operatorname{Max}^{a}$ & $\operatorname{Mean}^{b}$ & $\begin{array}{c}\text { Standard } \\
\text { deviation }^{b}\end{array}$ & Units \\
\hline \multirow[t]{4}{*}{$\mathrm{X} 701-21 \mathrm{G}$} & Gross alpha & $8 \mathrm{U}$ & $11 \mathrm{U}$ & NA & NA & $\mathrm{pCi} / \mathrm{L}$ \\
\hline & Gross beta & 15 & 27 & 20.0 & 5.1 & $\mathrm{pCi} / \mathrm{L}$ \\
\hline & Technetium & 26 & 36 & 32.0 & 4.5 & $\mathrm{pCi} / L$ \\
\hline & Total uranium & $1.0 \mathrm{U}$ & $1.0 \mathrm{U}$ & NA & NA & $\mu \mathrm{g} / \mathrm{L}$ \\
\hline \multirow[t]{4}{*}{$X 701-23 G$} & Gross alpha & $5 \mathrm{U}$ & $5 \mathrm{U}$ & NA & NA & $\mathrm{pCi} / \mathrm{L}$ \\
\hline & Gross beta & $9 \mathrm{U}$ & $11 \mathrm{U}$ & NA & NA & $\mathrm{pCi} / \mathrm{L}$ \\
\hline & Technetium & $22 \mathrm{U}$ & $22 \mathrm{U}$ & NA & NA & $\mathrm{pCi} / \mathrm{L}$ \\
\hline & Total uranium & $1.0 \mathrm{U}$ & $1.0 \mathrm{U}$ & NA & NA & $\mu \mathrm{g} / \mathrm{L}$ \\
\hline \multirow[t]{4}{*}{$X 701-24 G$} & Gross alpha & $5 \mathrm{U}$ & 26 & NA & NA & $\mathrm{pCi} / \mathrm{L}$ \\
\hline & Gross beta & 18 & 55 & 36.5 & 18.2 & $\mathrm{pCi} / \mathrm{L}$ \\
\hline & Technetium & 25 & 48 & 37.8 & 9.8 & $\mathrm{pCi} / \mathrm{L}$ \\
\hline & Total uranium & $1.0 \mathrm{U}$ & 6.8 & 2.9 & 2.9 & $\mu \mathrm{g} / \mathrm{L}$ \\
\hline \multirow[t]{4}{*}{$X 701-25 G$} & Gross alpha & $7 \mathrm{U}$ & $28 \mathrm{U}$ & NA & NA & $\mathrm{pCi} / \mathrm{L}$ \\
\hline & Gross beta & $10 \mathrm{U}$ & $38 \mathrm{U}$ & NA & NA & $\mathrm{pCi} / \mathrm{L}$ \\
\hline & Technetium & $22 \mathrm{U}$ & $24 \mathrm{U}$ & NA & NA & $\mathrm{pCi} / \mathrm{L}$ \\
\hline & Total uranium & $1.0 \mathrm{U}$ & $1.0 \mathrm{U}$ & NA & NA & $\mu \mathrm{g} / \mathrm{L}$ \\
\hline \multirow[t]{4}{*}{$X 701-30 G$} & Gross alpha & $6 \mathrm{U}$ & $7 \mathrm{U}$ & NA & NA & $\mathrm{pCi} / L$ \\
\hline & Gross beta & 10 & 16 & 11.4 & 4.6 & $\mathrm{pCi} / \mathrm{L}$ \\
\hline & Technetium & $22 \mathrm{U}$ & $24 \mathrm{U}$ & NA & NA & $\mathrm{pCi} / \mathrm{L}$ \\
\hline & Total uranium & $1.0 \mathrm{U}$ & $1.0 \mathrm{U}$ & NA & NA & $\mu \mathrm{g} / \mathrm{L}$ \\
\hline \multirow[t]{4}{*}{$X 701-31 G$} & Gross alpha & $4 U$ & $5 \mathrm{U}$ & NA & NA & $\mathrm{pCi} / \mathrm{L}$ \\
\hline & Gross beta & $9 \mathrm{U}$ & 13 & NA & NA & $\mathrm{pCi} / \mathrm{L}$ \\
\hline & Technetium & $22 \mathrm{U}$ & $22 \mathrm{U}$ & $\mathrm{NA}$ & NA & $\mathrm{pCi} / \mathrm{L}$ \\
\hline & Total uranium & $1.0 \mathrm{U}$ & $1.0 \mathrm{U}$ & $\mathrm{NA}$ & NA & $\mu \mathrm{g} / \mathrm{L}$ \\
\hline \multirow[t]{4}{*}{$X 701-32 G$} & Gross alpha & $5 \mathrm{U}$ & 27 & NA & NA & $\mathrm{pCi} / \mathrm{L}$ \\
\hline & Gross beta & $11 \mathrm{U}$ & $38 \mathrm{U}$ & NA & NA & $\mathrm{pCi} / \mathrm{L}$ \\
\hline & Technetium & $22 \mathrm{U}$ & $48 \mathrm{U}$ & NA & NA & $\mathrm{pCi} / \mathrm{L}$ \\
\hline & Total uranium & $1.0 \mathrm{U}$ & 3.3 & NA & NA & $\mu \mathrm{g} / \mathrm{L}$ \\
\hline \multirow[t]{4}{*}{ X701-38G } & Gross alpha & $6 \mathrm{U}$ & $7 \mathrm{U}$ & NA & NA & $\mathrm{pCi} / \mathrm{L}$ \\
\hline & Gross beta & $9 \mathrm{U}$ & $11 \mathrm{U}$ & NA & NA & $\mathrm{pCi} / \mathrm{L}$ \\
\hline & Technetium & $22 \mathrm{U}$ & 36 & NA & NA & $\mathrm{pCi} / \mathrm{L}$ \\
\hline & Total uranium & $1.0 \mathrm{U}$ & $1.0 \mathrm{U}$ & NA & NA & $\mu \mathrm{g} / \mathrm{L}$ \\
\hline \multirow[t]{4}{*}{$X 701-48 G$} & Gross alpha & $4 \mathrm{U}$ & 10 & 6.5 & 3.3 & $\mathrm{pCi} / \mathrm{L}$ \\
\hline & Gross beta & $9 \mathrm{U}$ & 16 & NA & NA & $\mathrm{pCi} / \mathrm{L}$ \\
\hline & Technetium & $22 \mathrm{U}$ & $24 \mathrm{U}$ & NA & NA & $\mathrm{pCi} / \mathrm{L}$ \\
\hline & Total uranium & $1.0 \mathrm{U}$ & 2.6 & NA & NA & $\mu \mathrm{g} / \mathrm{L}$ \\
\hline
\end{tabular}


Table 4.2 (continued)

\begin{tabular}{|c|c|c|c|c|c|c|}
\hline Well & Parameter & $\operatorname{Min}^{a}$ & $\operatorname{Max}^{a}$ & $\operatorname{Mean}^{b}$ & $\begin{array}{c}\text { Standard } \\
\text { deviation }^{b}\end{array}$ & Units \\
\hline \multirow[t]{4}{*}{ X701-49G } & Gross alpha & $6 U$ & 22 & 11.0 & 9.8 & $\mathrm{pCi} / \mathrm{L}$ \\
\hline & Gross beta & $9 \mathrm{U}$ & $11 \mathrm{U}$ & NA & NA & $\mathrm{pCi} / \mathrm{L}$ \\
\hline & Technetium & $22 \mathrm{U}$ & $22 \mathrm{U}$ & NA & NA & $\mathrm{pCi} / \mathrm{L}$ \\
\hline & Total uranium & 2.1 & 2.3 & 1.7 & 1.1 & $\mu \mathrm{g} / \mathrm{L}$ \\
\hline \multirow[t]{4}{*}{ X701-BW1G } & Gross alpha & $6 \mathrm{U}$ & $11 \mathrm{U}$ & NA & NA & $\mathrm{pCi} / \mathrm{L}$ \\
\hline & Gross beta & $9 \mathrm{U}$ & 35 & NA & NA & $\mathrm{pCi} / \mathrm{L}$ \\
\hline & Technetium & $22 \mathrm{U}$ & $22 U$ & NA & NA & $\mathrm{pCi} / \mathrm{L}$ \\
\hline & Total uranium & $1.0 \mathrm{U}$ & $1.0 \mathrm{U}$ & NA & NA & $\mu \mathrm{g} / \mathrm{L}$ \\
\hline \multirow[t]{4}{*}{ X701-BW4G } & Gross alpha & $8 \mathrm{U}$ & $11 \mathrm{U}$ & NA & NA & $\mathrm{pCi} / \mathrm{L}$ \\
\hline & Gross beta & $11 \mathrm{U}$ & 58 & NA & NA & $\mathrm{pCi} / \mathrm{L}$ \\
\hline & Technetium & $22 \mathrm{U}$ & 68 & NA & NA & $\mathrm{pCi} / \mathrm{L}$ \\
\hline & Total uranium & $1.0 \mathrm{U}$ & $1.0 \mathrm{U}$ & NA & NA & $\mu \mathrm{g} / \mathrm{L}$ \\
\hline \multirow[t]{4}{*}{ LBC-PZ03 } & Gross alpha & $5 \mathrm{U}$ & 13 & NA & NA & $\mathrm{pCi} / \mathrm{L}$ \\
\hline & Gross beta & 10 & $19 \mathrm{U}$ & NA & NA & $\mathrm{pCi} / \mathrm{L}$ \\
\hline & Technetium & $22 \mathrm{U}$ & $24 \mathrm{U}$ & NA & NA & $\mathrm{pCi} / \mathrm{L}$ \\
\hline & Total uranium & $1.0 \mathrm{U}$ & $1.0 \mathrm{U}$ & NA & NA & $\mu \mathrm{g} / \mathrm{L}$ \\
\hline \multirow[t]{4}{*}{ LBC-PZ06 } & Gross alpha & $4 \mathrm{U}$ & 37 & NA & NA & $\mathrm{pCi} / \mathrm{L}$ \\
\hline & Gross beta & $9 \mathrm{U}$ & $38 \mathrm{U}$ & NA & NA & $\mathrm{pCi} / \mathrm{L}$ \\
\hline & Technetium & $22 \mathrm{U}$ & 34 & NA & NA & $\mathrm{pCi} / \mathrm{L}$ \\
\hline & Total uranium & $1.0 \mathrm{U}$ & 5 & 2.4 & 1.9 & $\mu \mathrm{g} / \mathrm{L}$ \\
\hline \multicolumn{7}{|c|}{ Berea wells } \\
\hline \multirow[t]{4}{*}{$\mathrm{X701-50B}$} & Gross alpha & $18 \mathrm{U}$ & 41 & 26.3 & 13.5 & $\mathrm{pCi} / \mathrm{L}$ \\
\hline & Gross beta & 21 & 67 & 36.8 & 20.6 & $\mathrm{pCi} / \mathrm{L}$ \\
\hline & Technetium & $22 \mathrm{U}$ & 42 & NA & NA & $\mathrm{pCi} / \mathrm{L}$ \\
\hline & Total uranium & 1.0 & 4.3 & 2.2 & 1.5 & $\mu \mathrm{g} / \mathrm{L}$ \\
\hline \multirow[t]{4}{*}{ X701-58B } & Gross alpha & $11 \mathrm{U}$ & 31 & 19.9 & 12.7 & $\mathrm{pCi} / \mathrm{L}$ \\
\hline & Gross beta & $10 \mathrm{U}$ & 30 & NA & NA & $\mathrm{pCi} / \mathrm{L}$ \\
\hline & Technetium & $22 \mathrm{U}$ & $22 \mathrm{U}$ & NA & NA & $\mathrm{pCi} / \mathrm{L}$ \\
\hline & Total uranium & $1.0 \mathrm{U}$ & 2.5 & 1.5 & 0.8 & $\mu \mathrm{g} / \mathrm{L}$ \\
\hline \multirow[t]{3}{*}{$\mathrm{X} 701-61 \mathrm{~B}$} & Gross alpha & 48 & 92 & 67.3 & 18.6 & $\mathrm{pCi} / \mathrm{L}$ \\
\hline & Gross beta & 32 & 95 & 67.3 & 28.8 & $\mathrm{pCi} / \mathrm{L}$ \\
\hline & Technetium & $22 \mathrm{U}$ & $22 \mathrm{U}$ & $\mathrm{NA}$ & $\mathrm{NA}$ & $\mathrm{pCi} / \mathrm{L}$ \\
\hline - & Total uranium & 2.1 & 4.1 & 3.3 & 0.9 & $\mu \mathrm{g} / \mathrm{L}$ \\
\hline
\end{tabular}

${ }^{a}$ Qualifiers include: U-parameter was not detected; the result is the attainable dectection limit for the sample.

${ }^{b} \mathrm{NA}$-at least $50 \%$ of the results for the given parameter at this location were below detection; therefore, mean and standard deviation functions are not applicable. 
Table 4.3. Portsmouth groundwater monitoring for 1994: results for volatile organic compounds at X-749

\begin{tabular}{|c|c|c|c|c|c|c|}
\hline Well & Parameter ${ }^{a}$ & $\operatorname{Min}^{b}$ & $\operatorname{Max}^{b}$ & $\operatorname{Mean}^{c}$ & $\begin{array}{l}\text { Standard } \\
\text { deviation }^{c}\end{array}$ & Units \\
\hline \multicolumn{7}{|c|}{ Gallia wells } \\
\hline \multirow[t]{8}{*}{$\mathrm{X} 120-08 \mathrm{G}$} & $1,1,1-\mathrm{TCA}$ & 42 & 61 & 53 & 8 & $\mu \mathrm{g} / \mathrm{L}$ \\
\hline & 1,1-DCA & 11 & 16 & 13 & 2 & $\mu \mathrm{g} / \mathrm{L}$ \\
\hline & 1,1-DCE & 55 & 69 & 62 & 6 & $\mu \mathrm{g} / \mathrm{L}$ \\
\hline & 1,2-DCA & 3 & 4 & 3 & 1 & $\mu \mathrm{g} / \mathrm{L}$ \\
\hline & 1,2-DCEs (cis/t) & $2 \mathrm{U}$ & $2 U$ & NA & NA & $\mu \mathrm{g} / \mathrm{L}$ \\
\hline & Chloroform & $2 \mathrm{U}$ & $2 \mathrm{U}$ & NA & NA & $\mu \mathrm{g} / \mathrm{L}$ \\
\hline & Freon-113 & $2 \mathrm{U}$ & 5 & 4 & 2 & $\mu \mathrm{g} / \mathrm{L}$ \\
\hline & TCE & 9 & 10 & 9 & 1 & $\mu \mathrm{g} / \mathrm{L}$ \\
\hline \multirow[t]{8}{*}{ X749-04G } & 1,1,1-TCA & $10 \mathrm{U}$ & $20 \mathrm{U}$ & NA & NA & $\mu \mathrm{g} / \mathrm{L}$ \\
\hline & 1,1-DCA & $10 \mathrm{U}$ & $20 \mathrm{U}$ & NA & $\mathrm{NA}$ & $\mu \mathrm{g} / \mathrm{L}$ \\
\hline & 1,1-DCE & $10 \mathrm{U}$ & $20 \mathrm{U}$ & NA & NA & $\mu \mathrm{g} / \mathrm{L}$ \\
\hline & $1,2-\mathrm{DCA}$ & $10 \mathrm{U}$ & $20 \mathrm{U}$ & $\mathrm{NA}$ & $\mathrm{NA}$ & $\mu \mathrm{g} / \mathrm{L}$ \\
\hline & 1,2-DCEs $(c i s / t)$ & $10 U$ & $20 \mathrm{U}$ & NA & NA & $\mu \mathrm{g} / \mathrm{L}$ \\
\hline & Chloroform & $10 \mathrm{U}$ & $20 \mathrm{U}$ & NA & $\mathrm{NA}$ & $\mu \mathrm{g} / \mathrm{L}$ \\
\hline & Freon-113 & $10 \mathrm{U}$ & $20 \mathrm{U}$ & NA & NA & $\mu \mathrm{g} / \mathrm{L}$ \\
\hline & TCE & 55 & 180 & 117 & 53 & $\mu \mathrm{g} / \mathrm{L}$ \\
\hline \multirow[t]{8}{*}{ X749-06G } & 1,1,1-TCA & 3200 & 5140 & 4045 & 875 & $\mu \mathrm{g} / \mathrm{L}$ \\
\hline & 1,1-DCA & 1600 & 2690 & 2073 & 460 & $\mu \mathrm{g} / \mathrm{L}$ \\
\hline & 1,1-DCE & $100 \mathrm{U}$ & 2200 & 1393 & 940 & $\mu \mathrm{g} / \mathrm{L}$ \\
\hline & $1,2-\mathrm{DCA}$ & $100 \mathrm{U}$ & $1000 \mathrm{U}$ & NA & NA & $\mu \mathrm{g} / \mathrm{L}$ \\
\hline & 1,2-DCEs $(c i s / t)$ & 373 & $1000 \mathrm{U}$ & $\mathrm{NA}$ & NA & $\mu \mathrm{g} / \mathrm{L}$ \\
\hline & Chloroform & 182 & $1000 \mathrm{U}$ & NA & NA & $\mu \mathrm{g} / \mathrm{L}$ \\
\hline & Freon-113 & 511 & $1000 \mathrm{U}$ & 525 & 24 & $\mu \mathrm{g} / \mathrm{L}$ \\
\hline & $\mathrm{TCE}$ & 6200 & 10,600 & 8170 & 1941 & $\mu \mathrm{g} / \mathrm{L}$ \\
\hline \multirow[t]{8}{*}{ X749-07G } & $1,1,1-\mathrm{TCA}$ & 1370 & 2290 & 1668 & 420 & $\mu \mathrm{g} / \mathrm{L}$ \\
\hline & 1,1-DCA & 720 & 1040 & 840 & 139 & $\mu \mathrm{g} / \mathrm{L}$ \\
\hline & 1,1-DCE & 717 & 1140 & 842 & 200 & $\mu \mathrm{g} / \mathrm{L}$ \\
\hline & 1,2-DCA & 293 & 435 & 365 & 58 & $\mu \mathrm{g} / \mathrm{L}$ \\
\hline & 1,2-DCEs (cis/t) & $200 \mathrm{U}$ & 234 & NA & NA & $\mu \mathrm{g} / \mathrm{L}$ \\
\hline & Chloroform & $100 \mathrm{U}$ & $200 \mathrm{U}$ & NA & NA & $\mu \mathrm{g} / \mathrm{L}$ \\
\hline & Freon-113 & $200 U$ & 258 & NA & NA & $\mu \mathrm{g} / \mathrm{L}$ \\
\hline & TCE & 1590 & 2010 & 1728 & 191 & $\mu \mathrm{g} / \mathrm{L}$ \\
\hline \multirow[t]{4}{*}{ X749-08G } & $1,1,1-\mathrm{TCA}$ & 610 & 900 & 742 & 119 & $\mu \mathrm{g} / \mathrm{L}$ \\
\hline & 1,1-DCA & 21 & $40 \mathrm{U}$ & $\mathrm{NA}$ & NA & $\mu \mathrm{g} / \mathrm{L}$ \\
\hline & 1,1-DCE & 410 & 572 & 481.8 & 69.5 & $\mu \mathrm{g} / \mathrm{L}$ \\
\hline & 1,2-DCA & $20 \mathrm{U}$ & $40 \mathrm{U}$ & $\mathrm{NA}$ & $\mathrm{NA}$ & $\mu \mathrm{g} / \mathrm{L}$ \\
\hline
\end{tabular}


Table 4.3 (continued)

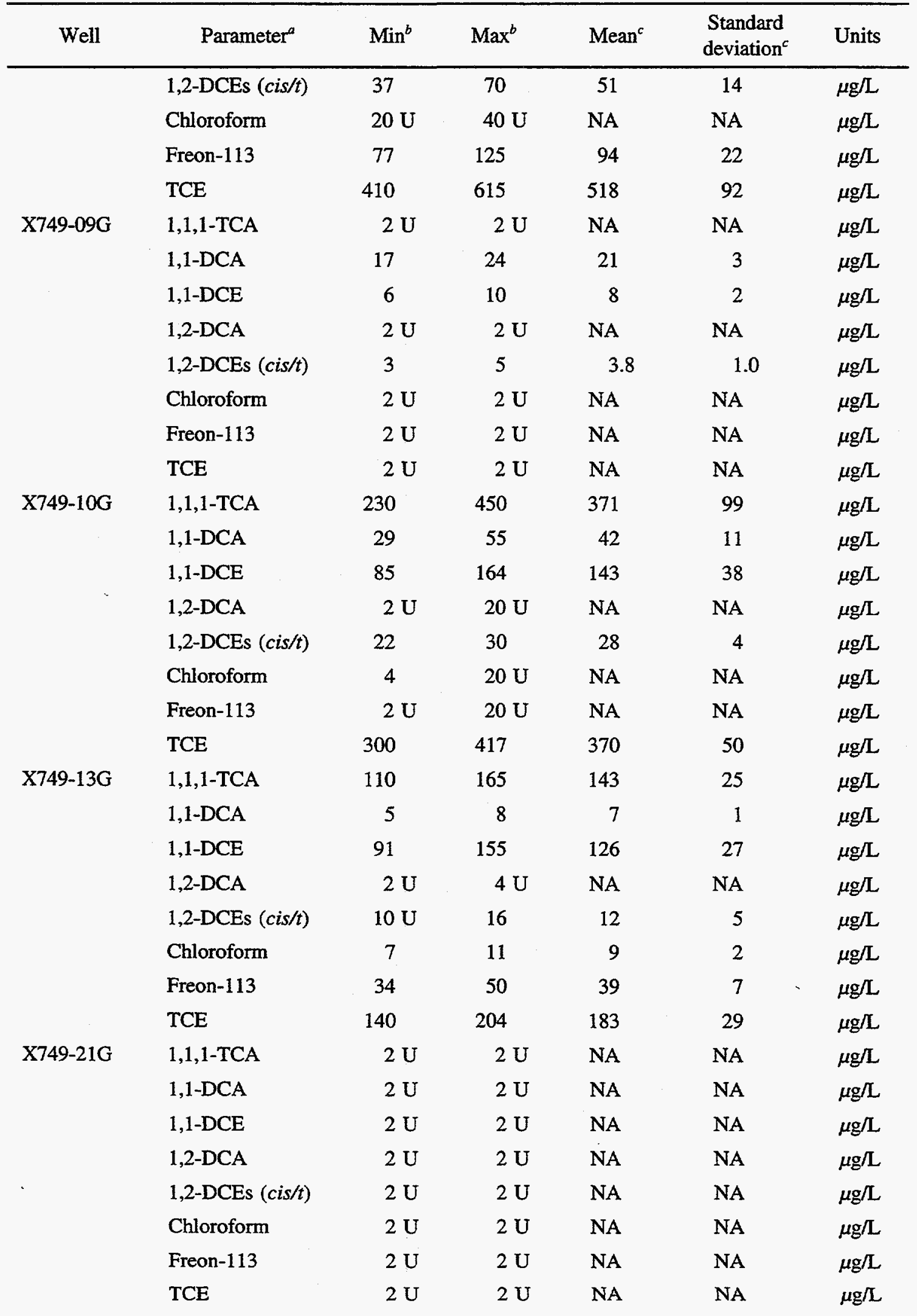


Table 4.3 (continued)

\begin{tabular}{|c|c|c|c|c|c|c|}
\hline Well & Parameter ${ }^{a}$ & $\operatorname{Min}^{b}$ & $\operatorname{Max}^{b}$ & $\operatorname{Mean}^{c}$ & $\begin{array}{l}\text { Standard } \\
\text { deviation }^{c}\end{array}$ & Units \\
\hline \multirow[t]{8}{*}{$X 749-23 G$} & $1,1,1-\mathrm{TCA}$ & $2 \mathrm{U}$ & $2 U$ & NA & NA & $\mu \mathrm{g} / \mathrm{L}$ \\
\hline & 1,1-DCA & $2 \mathrm{U}$ & $2 \mathrm{U}$ & NA & NA & $\mu \mathrm{g} / \mathrm{L}$ \\
\hline & 1,1-DCE & $2 U$ & $2 U$ & NA & NA & $\mu \mathrm{g} / \mathrm{L}$ \\
\hline & 1,2-DCA & $2 \mathrm{U}$ & $2 U$ & NA & NA & $\mu \mathrm{g} / \mathrm{L}$ \\
\hline & 1,2-DCEs (cis/t) & $2 \mathrm{U}$ & $2 \mathrm{U}$ & NA & NA & $\mu \mathrm{g} / \mathrm{L}$ \\
\hline & Chloroform & $2 U$ & $2 U$ & NA & NA & $\mu \mathrm{g} / \mathrm{L}$ \\
\hline & Freon-113 & $2 \mathrm{U}$ & $2 \mathrm{U}$ & NA & NA & $\mu \mathrm{g} / \mathrm{L}$ \\
\hline & TCE & $2 \mathrm{U}$ & $2 \mathrm{U}$ & NA & NA & $\mu \mathrm{g} / \mathrm{L}$ \\
\hline \multirow[t]{8}{*}{$X 749-24 G$} & 1,1,1-TCA & $2 \mathrm{U}$ & $2 \mathrm{U}$ & NA & NA & $\mu \mathrm{g} / \mathrm{L}$ \\
\hline & 1,1-DCA & $2 \mathrm{U}$ & $2 U$ & NA & NA & $\mu \mathrm{g} / \mathrm{L}$ \\
\hline & 1,1-DCE & $2 U$ & $2 U$ & NA & NA & $\mu \mathrm{g} / \mathrm{L}$ \\
\hline & $1,2-\mathrm{DCA}$ & $2 U$ & $2 \mathrm{U}$ & NA & NA & $\mu \mathrm{g} / \mathrm{L}$ \\
\hline & 1,2-DCEs $(c i s / t)$ & $2 \mathrm{U}$ & $2 \mathrm{U}$ & NA & NA & $\mu \mathrm{g} / \mathrm{L}$ \\
\hline & Chloroform & $2 \mathrm{U}$ & $2 U$ & NA & NA & $\mu \mathrm{g} / \mathrm{L}$ \\
\hline & Freon-113 & $2 U$ & $2 \mathrm{U}$ & $\mathrm{NA}$ & NA & $\mu \mathrm{g} / \mathrm{L}$ \\
\hline & TCE & $2 U$ & $2 U$ & NA & NA & $\mu \mathrm{g} / \mathrm{L}$ \\
\hline \multirow[t]{8}{*}{$X 749-25 G$} & $1,1,1-\mathrm{TCA}$ & 88 & 125 & 109 & 17 & $\mu \mathrm{g} / \mathrm{L}$ \\
\hline & 1,1-DCA & $2 \mathrm{U}$ & $20 \mathrm{U}$ & NA & NA & $\mu \mathrm{g} / \mathrm{L}$ \\
\hline & 1,1-DCE & 74 & 110 & 87 & 17 & $\mu \mathrm{g} / \mathrm{L}$ \\
\hline & $1,2-\mathrm{DCA}$ & $2 \mathrm{U}$ & $20 \mathrm{U}$ & NA & NA & $\mu \mathrm{g} / \mathrm{L}$ \\
\hline & 1,2-DCEs $(c i s / t)$ & 8 & $20 \mathrm{U}$ & NA & NA & $\mu \mathrm{g} / \mathrm{L}$ \\
\hline & Chloroform & 4 & $20 \mathrm{U}$ & NA & NA & $\mu \mathrm{g} / \mathrm{L}$ \\
\hline & Freon-113 & 24 & 40 & 32 & 7 & $\mu \mathrm{g} / \mathrm{L}$ \\
\hline & TCE & 126 & 160 & 140 & 16 & $\mu \mathrm{g} / \mathrm{L}$ \\
\hline \multirow[t]{8}{*}{$\mathrm{X} 749-26 \mathrm{G}$} & $1,1,1-\mathrm{TCA}$ & 183 & 249 & NA & NA & $\mu \mathrm{g} / \mathrm{L}$ \\
\hline & 1,1-DCA & 210 & 653 & 392 & 189 & $\mu \mathrm{g} / \mathrm{L}$ \\
\hline & 1,1-DCE & 119 & $200 \mathrm{U}$ & NA & NA & $\mu \mathrm{g} / \mathrm{L}$ \\
\hline & 1,2-DCA & 154 & 235 & NA & NA & $\mu \mathrm{g} / \mathrm{L}$ \\
\hline & 1,2-DCEs $(c i s / t)$ & $100 \mathrm{U}$ & $200 \mathrm{U}$ & NA & NA & $\mu \mathrm{g} / \mathrm{L}$ \\
\hline & Chloroform & $100 \mathrm{U}$ & $200 \mathrm{U}$ & NA & NA & $\mu \mathrm{g} / \mathrm{L}$ \\
\hline & Freon-113 & $100 \mathrm{U}$ & $200 \mathrm{U}$ & NA & NA & $\mu \mathrm{g} / \mathrm{L}$ \\
\hline & TCE & 360 & 1070 & 612 & 314 & $\mu \mathrm{g} / \mathrm{L}$ \\
\hline \multirow[t]{5}{*}{$X 749-32 G$} & $1,1,1-\mathrm{TCA}$ & 740 & 1380 & 1148 & 283 & $\mu \mathrm{g} / \mathrm{L}$ \\
\hline & $1,1-\mathrm{DCA}$ & 220 & 377 & 325 & 71 & $\mu \mathrm{g} / \mathrm{L}$ \\
\hline & 1,1-DCE & 540 & 860 & 746 & 142 & $\mu \mathrm{g} / \mathrm{L}$ \\
\hline & $1,2-\mathrm{DCA}$ & $100 \mathrm{U}$ & $200 \mathrm{U}$ & NA & NA & $\mu \mathrm{g} / \mathrm{L}$ \\
\hline & 1,2-DCEs $(c i s / t)$ & $100 \mathrm{U}$ & $200 \mathrm{U}$ & NA & NA & $\mu \mathrm{g} / \mathrm{L}$ \\
\hline
\end{tabular}


Table 4.3 (continued)

\begin{tabular}{|c|c|c|c|c|c|c|}
\hline Well & Parameter ${ }^{a}$ & $\operatorname{Min}^{b}$ & $\operatorname{Max}^{b}$ & Mean $^{c}$ & $\begin{array}{c}\text { Standard } \\
\text { deviation }^{c}\end{array}$ & Units \\
\hline \multirow{7}{*}{$X 749-36 G$} & Chloroform & $100 \mathrm{U}$ & $200 \mathrm{U}$ & NA & NA & $\mu \mathrm{g} / \mathrm{L}$ \\
\hline & Freon-113 & $200 \mathrm{U}$ & 300 & 236 & 92 & $\mu \mathrm{g} / \mathrm{L}$ \\
\hline & TCE & 1050 & 1870 & 1578 & 362 & $\mu \mathrm{g} / \mathrm{L}$ \\
\hline & $1,1,1-\mathrm{TCA}$ & 460 & 643 & 550 & 75 & $\mu \mathrm{g} / \mathrm{L}$ \\
\hline & 1,1-DCA & 110 & 137 & 124 & 12 & $\mu \mathrm{g} / \mathrm{L}$ \\
\hline & 1,1-DCE & 330 & 464 & 411.8 & 61.2 & $\mu \mathrm{g} / \mathrm{L}$ \\
\hline & 1,2-DCA & $20 \mathrm{U}$ & $40 \mathrm{U}$ & NA & NA & $\mu \mathrm{g} / \mathrm{L}$ \\
\hline \multirow{8}{*}{$\mathrm{X} 749-37 \mathrm{G}$} & 1,2-DCEs $(c i s / t)$ & $20 \mathrm{U}$ & $40 \mathrm{U}$ & NA & NA & $\mu \mathrm{g} / \mathrm{L}$ \\
\hline & Chloroform & $20 \mathrm{U}$ & $40 \mathrm{U}$ & NA & NA & $\mu \mathrm{g} / \mathrm{L}$ \\
\hline & Freon-113 & 60 & 89 & 74 & 13 & $\mu \mathrm{g} / \mathrm{L}$ \\
\hline & TCE & 270 & 346 & 307 & 33 & $\mu \mathrm{g} / \mathrm{L}$ \\
\hline & $1,1,1-\mathrm{TCA}$ & 371 & 840 & 531 & 211 & $\mu \mathrm{g} / \mathrm{L}$ \\
\hline & 1,1-DCA & 80 & 161 & 110 & 35 & $\mu \mathrm{g} / \mathrm{L}$ \\
\hline & 1,1-DCE & 275 & 560 & 399 & 118 & $\mu \mathrm{g} / \mathrm{L}$ \\
\hline & $1,2-\mathrm{DCA}$ & 15 & $40 \mathrm{U}$ & NA & NA & $\mu \mathrm{g} / \mathrm{L}$ \\
\hline \multirow{8}{*}{$X 749-43 G$} & 1,2-DCEs (cis/t) & $10 U$ & $40 \mathrm{U}$ & NA & NA & $\mu \mathrm{g} / \mathrm{L}$ \\
\hline & Chloroform & 16 & $40 \mathrm{U}$ & NA & NA & $\mu \mathrm{g} / \mathrm{L}$ \\
\hline & Freon-113 & 38 & 72 & 53 & 17 & $\mu \mathrm{g} / \mathrm{L}$ \\
\hline & TCE & 264 & 420 & 324 & 67 & $\mu \mathrm{g} / \mathrm{L}$ \\
\hline & $1,1,1-\mathrm{TCA}$ & $2 \mathrm{U}$ & $2 \mathrm{U}$ & NA & NA & $\mu \mathrm{g} / \mathrm{L}$ \\
\hline & 1,1-DCA & $2 U$ & $2 U$ & NA & NA & $\mu \mathrm{g} / \mathrm{L}$ \\
\hline & 1,1-DCE & $2 \mathrm{U}$ & $2 \mathrm{U}$ & NA & NA & $\mu \mathrm{g} / \mathrm{L}$ \\
\hline & $1,2-\mathrm{DCA}$ & $2 U$ & $2 \mathrm{U}$ & NA & NA & $\mu \mathrm{g} / \mathrm{L}$ \\
\hline \multirow{12}{*}{$X 749-44 G$} & 1,2-DCEs $(c i s / t)$ & $2 \mathrm{U}$ & $2 \mathrm{U}$ & NA & NA & $\mu \mathrm{g} / \mathrm{L}$ \\
\hline & Chloroform & $2 \mathrm{U}$ & $2 \mathrm{U}$ & NA & NA & $\mu \mathrm{g} / \mathrm{L}$ \\
\hline & Freon-113 & $2 U$ & $2 \mathrm{U}$ & NA & NA & $\mu \mathrm{g} / \mathrm{L}$ \\
\hline & TCE & $2 U$ & $2 U$ & NA & $\mathrm{NA}$ & $\mu \mathrm{g} / \mathrm{L}$ \\
\hline & 1,1,1-TCA & $2 \mathrm{U}$ & $2 U$ & NA & NA & $\mu \mathrm{g} / \mathrm{L}$ \\
\hline & 1,1-DCA & $2 U$ & $2 U$ & NA & NA & $\mu \mathrm{g} / \mathrm{L}$ \\
\hline & 1,1-DCE & $2 U$ & $2 \mathrm{U}$ & NA & NA & $\mu \mathrm{g} / \mathrm{L}$ \\
\hline & $1,2-\mathrm{DCA}$ & $2 U$ & $2 U$ & NA & $\mathrm{NA}$ & $\mu \mathrm{g} / \mathrm{L}$ \\
\hline & 1,2-DCEs $(c i s / t)$ & $2 U$ & $2 \mathrm{U}$ & NA & NA & $\mu \mathrm{g} / \mathrm{L}$ \\
\hline & Chloroform & $2 \mathrm{U}$ & $2 \mathrm{U}$ & NA & $\mathrm{NA}$ & $\mu \mathrm{g} / \mathrm{L}$ \\
\hline & Freon-113 & $2 U$ & $2 U$ & NA & $\mathrm{NA}$ & $\mu \mathrm{g} / \mathrm{L}$ \\
\hline & TCE & $2 \mathrm{U}$ & $2 U$ & $\mathrm{NA}$ & NA & $\mu \mathrm{g} / \mathrm{L}$ \\
\hline
\end{tabular}


Table 4.3 (continued)

\begin{tabular}{|c|c|c|c|c|c|c|}
\hline Well & Parameter & $\operatorname{Min}^{b}$ & $\operatorname{Max}^{b}$ & Mean $^{c}$ & $\begin{array}{l}\text { Standard } \\
\text { deviation }^{c}\end{array}$ & Units \\
\hline \multirow[t]{8}{*}{$X 749-45 G$} & $1,1,1-\mathrm{TCA}$ & $2 U$ & $2 \mathrm{U}$ & NA & NA & $\mu \mathrm{g} / \mathrm{L}$ \\
\hline & 1,1-DCA & $2 U$ & $2 \mathrm{U}$ & NA & NA & $\mu \mathrm{g} / \mathrm{L}$ \\
\hline & 1,1-DCE & $2 \mathrm{U}$ & $2 \mathrm{U}$ & NA & NA & $\mu \mathrm{g} / \mathrm{L}$ \\
\hline & 1,2-DCA & $2 \mathrm{U}$ & $2 \mathrm{U}$ & NA & NA & $\mu \mathrm{g} / \mathrm{L}$ \\
\hline & 1,2-DCEs (cis/t) & $2 \mathrm{U}$ & $2 \mathrm{U}$ & NA & NA & $\mu \mathrm{g} / \mathrm{L}$ \\
\hline & Chloroform & $2 U$ & $2 U$ & NA & NA & $\mu \mathrm{g} / \mathrm{L}$ \\
\hline & Freon-113 & $2 \mathrm{U}$ & 3 & NA & NA & $\mu \mathrm{g} / \mathrm{L}$ \\
\hline & TCE & 2 & 7 & 4 & 2 & $\mu \mathrm{g} / \mathrm{L}$ \\
\hline \multirow[t]{8}{*}{ X749-PZ02G } & 1,1,1-TCA & $2 \mathrm{U}$ & $2 \mathrm{U}$ & NA & NA & $\mu \mathrm{g} / \mathrm{L}$ \\
\hline & 1,1-DCA & $2 \mathrm{U}$ & $2 \mathrm{U}$ & $\mathrm{NA}$ & NA & $\mu \mathrm{g} / \mathrm{L}$ \\
\hline & 1,1-DCE & $2 \mathrm{U}$ & $2 \mathrm{U}$ & NA & NA & $\mu \mathrm{g} / \mathrm{L}$ \\
\hline & 1,2-DCA & $2 U$ & $2 \mathrm{U}$ & NA & NA & $\mu \mathrm{g} / \mathrm{L}$ \\
\hline & 1,2-DCEs $(c i s / t)$ & $2 U$ & $2 \mathrm{U}$ & NA & NA & $\mu \mathrm{g} / \mathrm{L}$ \\
\hline & Chloroform & $2 \mathrm{U}$ & $2 \mathrm{U}$ & NA & NA & $\mu \mathrm{g} / \mathrm{L}$ \\
\hline & Freon-113 & $2 \mathrm{U}$ & $2 \mathrm{U}$ & $\mathrm{NA}$ & NA & $\mu \mathrm{g} / \mathrm{L}$ \\
\hline & TCE & $2 \mathrm{U}$ & $2 \mathrm{U}$ & $\mathrm{NA}$ & NA & $\mu \mathrm{g} / \mathbb{L}$ \\
\hline \multirow[t]{8}{*}{ X749-PZ03G } & $1,1,1-\mathrm{TCA}$ & $2 \mathrm{U}$ & $2 \mathrm{U}$ & NA & NA & $\mu \mathrm{g} / \mathrm{L}$ \\
\hline & 1,1-DCA & $2 \mathrm{U}$ & $2 \mathrm{U}$ & $\mathrm{NA}$ & NA & $\mu \mathrm{g} / \mathrm{L}$ \\
\hline & 1,1-DCE & $2 \mathrm{U}$ & $2 \mathrm{U}$ & $\mathrm{NA}$ & NA & $\mu \mathrm{g} / \mathrm{L}$ \\
\hline & $1,2-\mathrm{DCA}$ & $2 U$ & $2 \mathrm{U}$ & NA & NA & $\mu \mathrm{g} / \mathrm{L}$ \\
\hline & 1,2-DCEs $(c i s / t)$ & $2 \mathrm{U}$ & $2 U$ & NA & NA & $\mu \mathrm{g} / \mathrm{L}$ \\
\hline & Chloroform & $2 \mathrm{U}$ & $2 \mathrm{U}$ & $\mathrm{NA}$ & NA & $\mu \mathrm{g} / \mathrm{L}$ \\
\hline & Freon-113 & $2 \mathrm{U}$ & $2 U$ & $\mathrm{NA}$ & NA & $\mu \mathrm{g} / \mathrm{L}$ \\
\hline & TCE & $2 U$ & $2 U$ & NA & NA & $\mu \mathrm{g} / \mathrm{L}$ \\
\hline \multirow[t]{8}{*}{ X749-PZ04G } & 1,1,1-TCA & 14 & 22 & 15 & 5 & $\mu \mathrm{g} / \mathrm{L}$ \\
\hline & 1,1-DCA & 18 & 33 & 24 & 7 & $\mu \mathrm{g} / \mathrm{L}$ \\
\hline & 1,1-DCE & 14 & 22 & NA & NA & $\mu \mathrm{g} / \mathrm{L}$ \\
\hline & $1,2-\mathrm{DCA}$ & 6 & $20 \mathrm{U}$ & NA & NA & $\mu \mathrm{g} / \mathrm{L}$ \\
\hline & 1,2-DCEs (cis/t) & 8 & $20 \mathrm{U}$ & 10 & 2 & $\mu \mathrm{g} / \mathrm{L}$ \\
\hline & Chloroform & 5 & $20 \mathrm{U}$ & 7 & 2 & $\mu \mathrm{g} / \mathrm{L}$ \\
\hline & Freon-113 & 31 & 56 & 39 & 12 & $\mu \mathrm{g} / \mathrm{L}$ \\
\hline & TCE & 102 & 160 & 124 & 26 & $\mu \mathrm{g} / \mathrm{L}$ \\
\hline \multirow[t]{5}{*}{ X749-PZ05G } & $1,1,1-\mathrm{TCA}$ & $2 \mathrm{U}$ & $2 \mathrm{U}$ & NA & NA & $\mu \mathrm{g} / \mathrm{L}$ \\
\hline & 1,1-DCA & $2 \mathrm{U}$ & $2 \mathrm{U}$ & NA & NA & $\mu \mathrm{g} / \mathrm{L}$ \\
\hline & 1,1-DCE & $2 \mathrm{U}$ & $2 \mathrm{U}$ & NA & NA & $\mu \mathrm{g} / \mathrm{L}$ \\
\hline & 1,2-DCA & $2 U$ & $2 \mathrm{U}$ & NA & NA & $\mu \mathrm{g} / \mathrm{L}$ \\
\hline & 1,2-DCEs (cis/t) & $2 U$ & $2 U$ & NA & NA & $\mu \mathrm{g} / \mathrm{L}$ \\
\hline
\end{tabular}


Table 4.3 (continued)

\begin{tabular}{|c|c|c|c|c|c|c|}
\hline Well & Parameter ${ }^{a}$ & $\operatorname{Min}^{b}$ & $\operatorname{Max}^{b}$ & $\operatorname{Mean}^{c}$ & $\begin{array}{c}\text { Standard } \\
\text { deviation }^{c}\end{array}$ & Units \\
\hline & Chloroform & $2 \mathrm{U}$ & $2 \mathrm{U}$ & NA & NA & $\mu \mathrm{g} / \mathrm{L}$ \\
\hline & Freon-113 & $2 U$ & $2 U$ & NA & NA & $\mu \mathrm{g} / \mathrm{L}$ \\
\hline & TCE & $2 \mathrm{U}$ & $2 U$ & NA & NA & $\mu \mathrm{g} / \mathrm{L}$ \\
\hline \multicolumn{7}{|c|}{ Berea wells } \\
\hline \multirow[t]{8}{*}{ X749-14B } & $1,1,1-\mathrm{TCA}$ & $2 \mathrm{U}$ & $2 \mathrm{U}$ & NA & NA & $\mu \mathrm{g} / \mathrm{L}$ \\
\hline & 1,1-DCA & $2 \mathrm{U}$ & $2 U$ & NA & NA & $\mu \mathrm{g} / \mathrm{L}$ \\
\hline & 1,1-DCE & $2 \mathrm{U}$ & $2 \mathrm{U}$ & NA & NA & $\mu \mathrm{g} / \mathrm{L}$ \\
\hline & 1,2-DCA & $2 \mathrm{U}$ & $2 \mathrm{U}$ & NA & NA & $\mu \mathrm{g} / \mathrm{L}$ \\
\hline & 1,2-DCEs (cis/t) & $2 \mathrm{U}$ & $2 \mathrm{U}$ & NA & NA & $\mu \mathrm{g} / \mathrm{L}$ \\
\hline & Chloroform & $2 U$ & $2 \mathrm{U}$ & NA & NA & $\mu \mathrm{g} / \mathrm{L}$ \\
\hline & Freon-113 & $2 \mathrm{U}$ & $2 \mathrm{U}$ & NA & NA & $\mu \mathrm{g} / \mathrm{L}$ \\
\hline & TCE & $2 \mathrm{U}$ & $2 \mathrm{U}$ & $\mathrm{NA}$ & NA & $\mu \mathrm{g} / \mathrm{L}$ \\
\hline \multirow[t]{8}{*}{ X749-50B } & $1,1,1-\mathrm{TCA}$ & $2 \mathrm{U}$ & $2 \mathrm{U}$ & NA & NA & $\mu \mathrm{g} / \mathrm{L}$ \\
\hline & 1,1-DCA & 4 & 6 & 5 & 1 & $\mu \mathrm{g} / \mathrm{L}$ \\
\hline & 1,1-DCE & $2 \mathrm{U}$ & $2 \mathrm{U}$ & NA & NA & $\mu \mathrm{g} / \mathrm{L}$ \\
\hline & 1,2-DCA & 3 & 4 & 3 & 1 & $\mu \mathrm{g} / \mathrm{L}$ \\
\hline & 1,2-DCEs (cis/t) & $2 \mathrm{U}$ & $2 \mathrm{U}$ & NA & NA & $\mu \mathrm{g} / \mathrm{L}$ \\
\hline & Chloroform & $2 \mathrm{U}$ & $2 \mathrm{U}$ & NA & NA & $\mu \mathrm{g} / \mathrm{L}$ \\
\hline & Freon-113 & $2 \mathrm{U}$ & $2 \mathrm{U}$ & NA & NA & $\mu \mathrm{g} / \mathrm{L}$ \\
\hline & TCE & $2 \mathrm{U}$ & $2 \mathrm{U}$ & NA & NA & $\mu \mathrm{g} / \mathrm{L}$ \\
\hline \multirow[t]{8}{*}{ X749-51B } & $1,1,1-\mathrm{TCA}$ & $2 U$ & $2 \mathrm{U}$ & NA & NA & $\mu \mathrm{g} / \mathrm{L}$ \\
\hline & 1,1-DCA & $2 \mathrm{U}$ & $2 \mathrm{U}$ & NA & NA & $\mu \mathrm{g} / \mathrm{L}$ \\
\hline & 1,1-DCE & $2 U$ & $2 \mathrm{U}$ & NA & NA & $\mu \mathrm{g} / \mathrm{L}$ \\
\hline & 1,2-DCA & $2 \mathrm{U}$ & $2 \mathrm{U}$ & NA & NA & $\mu \mathrm{g} / \mathrm{L}$ \\
\hline & 1,2-DCEs (cis/t) & $2 \mathrm{U}$ & $2 U$ & NA & NA & $\mu \mathrm{g} / \mathrm{L}$ \\
\hline & Chloroform & $2 \mathrm{U}$ & $2 \mathrm{U}$ & NA & NA & $\mu \mathrm{g} / \mathrm{L}$ \\
\hline & Freon-113 & $2 \mathrm{U}$ & $2 \mathrm{U}$ & NA & NA & $\mu \mathrm{g} / \mathrm{L}$ \\
\hline & TCE & $2 \mathrm{U}$ & $2 \mathrm{U}$ & NA & NA & $\mu \mathrm{g} / \mathrm{L}$ \\
\hline \multirow[t]{8}{*}{ X749-54B } & $1,1,1-\mathrm{TCA}$ & $2 \mathrm{U}$ & $2 \mathrm{U}$ & NA & NA & $\mu \mathrm{g} / \mathrm{L}$ \\
\hline & 1,1-DCA & $2 U$ & $2 \mathrm{U}$ & NA & NA & $\mu \mathrm{g} / \mathrm{L}$ \\
\hline & $1,1-\mathrm{DCE}$ & $2 \mathrm{U}$ & $2 U$ & NA & NA & $\mu \mathrm{g} / \mathrm{L}$ \\
\hline & 1,2-DCA & $2 \mathrm{U}$ & 4 & NA & NA & $\mu \mathrm{g} / \mathrm{L}$ \\
\hline & 1,2-DCEs $(c i s / t)$ & $2 \mathrm{U}$ & $2 U$ & NA & $\mathrm{NA}$ & $\mu \mathrm{g} / \mathrm{L}$ \\
\hline & Chloroform & $2 U$ & $2 \mathrm{U}$ & NA & NA & $\mu \mathrm{g} / \mathrm{L}$ \\
\hline & Freon-113 & $2 \mathrm{U}$ & $2 \mathrm{U}$ & NA & NA & $\mu \mathrm{g} / \mathrm{L}$ \\
\hline & TCE & $2 \mathrm{U}$ & $2 \mathrm{U}$ & NA & NA & $\mu \mathrm{g} / \mathrm{L}$ \\
\hline
\end{tabular}


Table 4.3 (continued)

\begin{tabular}{cllclll}
\hline Well & \multicolumn{1}{c}{ Parameter $^{a}$} & Min $^{b}$ & Max $^{b}$ & Mean $^{c}$ & $\begin{array}{c}\text { Standard } \\
\text { deviation }^{c}\end{array}$ & Units \\
\hline X749-60B & $1,1,1-\mathrm{TCA}$ & $2 \mathrm{U}$ & $2 \mathrm{U}$ & $\mathrm{NA}$ & $\mathrm{NA}$ & $\mu \mathrm{g} / \mathrm{L}$ \\
& $1,1-\mathrm{DCA}$ & $2 \mathrm{U}$ & $2 \mathrm{U}$ & $\mathrm{NA}$ & NA & $\mu \mathrm{g} / \mathrm{L}$ \\
& $1,1-\mathrm{DCE}$ & $2 \mathrm{U}$ & $2 \mathrm{U}$ & NA & NA & $\mu \mathrm{g} / \mathrm{L}$ \\
& $1,2-\mathrm{DCA}$ & $2 \mathrm{U}$ & $2 \mathrm{U}$ & NA & NA & $\mu \mathrm{g} / \mathrm{L}$ \\
& $1,2-\mathrm{DCEs}($ cis/t) & $2 \mathrm{U}$ & $2 \mathrm{U}$ & NA & NA & $\mu \mathrm{g} / \mathrm{L}$ \\
& Chloroform & $2 \mathrm{U}$ & $2 \mathrm{U}$ & NA & NA & $\mu \mathrm{g} / \mathrm{L}$ \\
& Freon-113 & $2 \mathrm{U}$ & $2 \mathrm{U}$ & NA & NA & $\mu \mathrm{g} / \mathrm{L}$ \\
& TCE & $2 \mathrm{U}$ & $2 \mathrm{U}$ & NA & NA & $\mu \mathrm{g} / \mathrm{L}$ \\
& $1,1,1-\mathrm{TCA}$ & $2 \mathrm{U}$ & $2 \mathrm{U}$ & NA & NA & $\mu \mathrm{g} / \mathrm{L}$ \\
& $1,1-\mathrm{DCA}$ & $2 \mathrm{U}$ & $2 \mathrm{U}$ & NA & NA & $\mu \mathrm{g} / \mathrm{L}$ \\
& $1,1-\mathrm{DCE}$ & $2 \mathrm{U}$ & $2 \mathrm{U}$ & NA & NA & $\mu \mathrm{g} / \mathrm{L}$ \\
& $1,2-\mathrm{DCA}$ & $2 \mathrm{U}$ & $2 \mathrm{U}$ & NA & NA & $\mu \mathrm{g} / \mathrm{L}$ \\
& $1,2-\mathrm{DCEs}($ cis $/ t)$ & $2 \mathrm{U}$ & $2 \mathrm{U}$ & NA & NA & $\mu \mathrm{g} / \mathrm{L}$ \\
& Chloroform & $2 \mathrm{U}$ & $2 \mathrm{U}$ & NA & NA & $\mu \mathrm{g} / \mathrm{L}$ \\
& Freon-113 & $2 \mathrm{U}$ & $2 \mathrm{U}$ & NA & NA & $\mu \mathrm{g} / \mathrm{L}$ \\
& TCE & $2 \mathrm{U}$ & $2 \mathrm{U}$ & NA & NA & $\mu \mathrm{g} / \mathrm{L}$ \\
\hline
\end{tabular}

${ }^{a}$ Parameter abbreviations: 1,1,1-TCA $=1,1,1$-Trichloroethane; 1,1-DCA = 1,1-Dichloroethane; 1,1-DCE $=1,1$-Dichloroethene; 1,2 -DCA $=1,2$-Dichloroethane; 1,2 -DCEs $(c i s / t)=1,2-$

Dichloroethenes (cis and trans); and TCE = Trichloroethylene.

${ }^{b}$ Qualifers include: U-parameter was not detected; the result is the attainable detection limit for the sample.

NA-at least $50 \%$ of the results for the given parameter at this location were below detection; therefore, mean and standard deviation functions are not applicable. 
Table 4.4. Portsmouth groundwater monitoring for 1994: results for radionuclide parameters at X-749

\begin{tabular}{|c|c|c|c|c|c|c|}
\hline Well & Parameter & $\operatorname{Min}^{a}$ & $\operatorname{Max}^{a}$ & $\operatorname{Mean}^{b}$ & $\begin{array}{c}\text { Standard } \\
\text { deviation }^{b}\end{array}$ & Units \\
\hline \multicolumn{7}{|c|}{ Gallia wells } \\
\hline \multirow[t]{4}{*}{$\mathrm{X} 120-08 \mathrm{G}$} & Gross alpha & $5 \mathrm{U}$ & 13 & NA & NA & $\mathrm{pCi} / \mathrm{L}$ \\
\hline & Gross beta & $9 U$ & 25 & NA & NA & $\mathrm{pCi} / \mathrm{L}$ \\
\hline & Technetium & $22 \mathrm{U}$ & $22 \mathrm{U}$ & NA & NA & $\mathrm{pCi} / \mathrm{L}$ \\
\hline & Total uranium & 2.9 & 7.5 & 4 & 2.2 & $\mu g / \mathrm{L}$ \\
\hline \multirow[t]{4}{*}{ X749-04G } & Gross alpha & $5 \mathrm{U}$ & 8 & NA & NA & $\mathrm{pCi} / \mathrm{L}$ \\
\hline & Gross beta & $91 \mathrm{U}$ & $11 \mathrm{U}$ & NA & NA & $\mathrm{pCi} / \mathrm{L}$ \\
\hline & Technetium & $22 \mathrm{U}$ & $22 \mathrm{U}$ & NA & NA & $\mathrm{pCi} / \mathrm{L}$ \\
\hline & Total uranium & $1.0 \mathrm{U}$ & 1.6 & NA & NA & $\mu \mathrm{g} / \mathrm{L}$ \\
\hline \multirow[t]{4}{*}{ X749-06G } & Gross alpha & $6 \mathrm{U}$ & 9 & $\mathrm{NA}$ & NA & $\mathrm{pCi} / \mathrm{L}$ \\
\hline & Gross beta & 66 & 90 & 77 & 10 & $\mathrm{pCi} / \mathrm{L}$ \\
\hline & Technetium & 104 & 136 & 121 & 13 & $\mathrm{pCi} / \mathrm{L}$ \\
\hline & Total uranium & $1.0 \mathrm{U}$ & 4.1 & NA & NA & $\mu \mathrm{g} / \mathrm{L}$ \\
\hline \multirow[t]{4}{*}{ X749-07G } & Gross alpha & $6 \mathrm{U}$ & 14 & 8 & 4.6 & $\mathrm{pCi} / \mathrm{L}$ \\
\hline & Gross beta & 187 & 382 & 241 & 94 & $\mathrm{pCi} / \mathrm{L}$ \\
\hline & Technetium & 288 & 448 & 335 & 76 & $\mathrm{pCi} / \mathrm{L}$ \\
\hline & Total uranium & $1.0 \mathrm{U}$ & 2.3 & NA & NA & $\mu \mathrm{g} / \mathrm{L}$ \\
\hline \multirow[t]{4}{*}{ X749-08G } & Gross alpha & $6 \mathrm{U}$ & 8 & $\mathbf{N A}$ & NA & $\mathrm{pCi} / \mathrm{L}$ \\
\hline & Gross beta & 144 & 164 & 152 & 8.8 & $\mathrm{pCi} / \mathrm{L}$ \\
\hline & Technetium & 173 & 304 & 218 & 59 & $\mathrm{pCi} / \mathrm{L}$ \\
\hline & Total uranium & $1.0 \mathrm{U}$ & 1.2 & NA & NA & $\mu \mathrm{g} / \mathrm{L}$ \\
\hline \multirow[t]{4}{*}{$\mathrm{X749-09G}$} & Gross alpha & $5 \mathrm{U}$ & 9 & NA & NA & $\mathrm{pCi} / \mathrm{L}$ \\
\hline & Gross beta & $9 \mathrm{U}$ & $11 \mathrm{U}$ & NA & NA & $\mathrm{pCi} / \mathrm{L}$ \\
\hline & Technetium & $22 \mathrm{U}$ & $22 \mathrm{U}$ & NA & NA & $\mathrm{pCi} / \mathrm{L}$ \\
\hline & Total uranium & $1.0 \mathrm{U}$ & 2.6 & NA & NA & $\mu \mathrm{g} / \mathrm{L}$ \\
\hline \multirow[t]{4}{*}{ X749-10G } & Gross alpha & $8 \mathrm{U}$ & 39 & NA & NA & $\mathrm{pCi} / \mathrm{L}$ \\
\hline & Gross beta & 14 & 53 & 25 & 19 & $\mathrm{pCi} / \mathrm{L}$ \\
\hline & Technetium & $22 U$ & 25 & 22 & 7.0 & $\mathrm{pCi} / \mathrm{L}$ \\
\hline & Total uranium & 1.4 & 3.3 & 2 & 0.9 & $\mu \mathrm{g} / \mathrm{L}$ \\
\hline \multirow[t]{4}{*}{$X 749-13 G$} & Gross alpha & $5 \mathrm{U}$ & 8 & NA & NA & $\mathrm{pCi} / \mathrm{L}$ \\
\hline & Gross beta & 104 & 129 & 117 & 10 & $\mathrm{pCi} / \mathrm{L}$ \\
\hline & Technetium & 143 & 193 & 160 & 23 & $\mathrm{pCi} / \mathrm{L}$ \\
\hline & Total uranium & $1.0 \mathrm{U}$ & $1.0 \mathrm{U}$ & NA & NA & $\mu \mathrm{g} / \mathrm{L}$ \\
\hline \multirow[t]{4}{*}{$\mathrm{X} 749-21 \mathrm{G}$} & Gross alpha & $5 \mathrm{U}$ & 50 & NA & NA & $\mathrm{pCi} / \mathrm{L}$ \\
\hline & Gross beta & $9 \mathrm{U}$ & 53 & NA & NA & $\mathrm{pCi} / \mathrm{L}$ \\
\hline & Technetium & $22 \mathrm{U}$ & $22 \mathrm{U}$ & NA & NA & $\mathrm{pCi} / \mathrm{L}$ \\
\hline & Total uranium & $1.0 \mathrm{U}$ & 13.2 & 6 & 5.8 & $\mu \mathrm{g} / \mathrm{L}$ \\
\hline \multirow[t]{4}{*}{$\mathrm{X} 749-23 \mathrm{G}$} & Gross alpha & $5 \mathrm{U}$ & 10 & NA & NA & $\mathrm{pCi} / \mathrm{L}$ \\
\hline & Gross beta & $9 \mathrm{U}$ & $11 \mathrm{U}$ & NA & NA & $\mathrm{pCi} / \mathrm{L}$ \\
\hline & Technetium & $22 \mathrm{U}$ & $22 \mathrm{U}$ & NA & NA & $\mathrm{pCi} / \mathrm{L}$ \\
\hline & Total uranium & $1.0 \mathrm{U}$ & 1.6 & NA & NA & $\mu \mathrm{g} / \mathrm{L}$ \\
\hline
\end{tabular}


Table 4.4 (continued)

\begin{tabular}{|c|c|c|c|c|c|c|}
\hline Well & Parameter & $\operatorname{Min}^{a}$ & $\operatorname{Max}^{a}$ & $\operatorname{Mean}^{b}$ & $\begin{array}{c}\text { Standard } \\
\text { deviation }^{b}\end{array}$ & Units \\
\hline \multirow[t]{4}{*}{$\mathrm{X} 749-24 \mathrm{G}$} & Gross alpha & $4 \mathrm{U}$ & 6 & NA & NA & $\mathrm{pCi} / \mathrm{L}$ \\
\hline & Gross beta & $9 \mathrm{U}$ & $11 \mathrm{U}$ & NA & NA & $\mathrm{pCi} / \mathrm{L}$ \\
\hline & Technetium & $22 \mathrm{U}$ & $22 \mathrm{U}$ & NA & NA & $\mathrm{pCi} / \mathrm{L}$ \\
\hline & Total uranium & $1.0 \mathrm{U}$ & $1.0 \mathrm{U}$ & NA & NA & $\mu \mathrm{g} / \mathrm{L}$ \\
\hline \multirow[t]{4}{*}{$X 749-25 G$} & Gross alpha & $5 \mathrm{U}$ & $6 \mathrm{U}$ & NA & NA & $\mathrm{pCi} / \mathrm{L}$ \\
\hline & Gross beta & 88 & 104 & 97 & 6.7 & $\mathrm{pCi} / \mathrm{L}$ \\
\hline & Technetium & 107 & 172 & 136 & 29 & $\mathrm{pCi} / \mathrm{L}$ \\
\hline & Total uranium & $1.0 \mathrm{U}$ & $1.0 \mathrm{U}$ & NA & NA & $\mu \mathrm{g} / \mathrm{L}$ \\
\hline \multirow[t]{4}{*}{$X 749-26 G$} & Gross alpha & $4 \mathrm{U}$ & $5 \mathrm{U}$ & NA & NA & $\mathrm{pCi} / \mathrm{L}$ \\
\hline & Gross beta & 108 & 328 & 214 & 97 & $\mathrm{pCi} / \mathrm{L}$ \\
\hline & Technetium & 153 & 445 & 303 & 137 & $\mathrm{pCi} / \mathrm{L}$ \\
\hline & Total uranium & $1.0 \mathrm{U}$ & $1.0 \mathrm{U}$ & NA & NA & $\mu \mathrm{g} / \mathrm{L}$ \\
\hline \multirow[t]{4}{*}{$X 749-32 G$} & Gross alpha & 5 & 10 & $\mathrm{NA}$ & NA & $\mathrm{pCi} / \mathrm{L}$ \\
\hline & Gross beta & 166 & 213 & 189 & 21 & $\mathrm{pCi} / \mathrm{L}$ \\
\hline & Technetium & 227 & 298 & 261 & 34 & $\mathrm{pCi} / L$ \\
\hline & Total uranium & $1.0 \mathrm{U}$ & $1.0 \mathrm{U}$ & NA & NA & $\mu \mathrm{g} / \mathrm{L}$ \\
\hline \multirow[t]{4}{*}{ X749-36G } & Gross alpha & $2 \mathrm{U}$ & $6 \mathrm{U}$ & $\mathrm{NA}$ & $\mathrm{NA}$ & $\mathrm{pCi} / \mathrm{L}$ \\
\hline & Gross beta & 14 & 18 & 16 & 1.7 & $\mathrm{pCi} / \mathrm{L}$ \\
\hline & Technetium & $22 \mathrm{U}$ & 34 & 24 & 10 & $\mathrm{pCi} / \mathrm{L}$ \\
\hline & Total uranium & $1.0 \mathrm{U}$ & $1.0 \mathrm{U}$ & $\mathrm{NA}$ & $\mathrm{NA}$ & $\mu \mathrm{g} / \mathrm{L}$ \\
\hline \multirow[t]{4}{*}{$\mathrm{X} 749-37 \mathrm{G}$} & Gross alpha & $4 \mathrm{U}$ & $5 \mathrm{U}$ & NA & NA & $\mathrm{pCi} / \mathrm{L}$ \\
\hline & Gross beta & $9 \mathrm{U}$ & 33 & 16 & 12 & $\mathrm{pCi} / \mathrm{L}$ \\
\hline & Technetium & $24 U$ & 36 & 27 & 10 & $\mathrm{pCi} / \mathrm{L}$ \\
\hline & Total uranium & $1.0 \mathrm{U}$ & $1.0 \mathrm{U}$ & NA & NA & $\mu \mathrm{g} / \mathrm{L}$ \\
\hline \multirow[t]{4}{*}{$X 749-43 G$} & Gross alpha & $5 \mathrm{U}$ & 11 & $\mathrm{NA}$ & $\mathrm{NA}$ & $\mathrm{pCi} / \mathrm{L}$ \\
\hline & Gross beta & 10 & 21 & 15 & 4.6 & $\mathrm{pCi} / \mathrm{L}$ \\
\hline & Technetium & $22 \mathrm{U}$ & 36 & NA & NA & $\mathrm{pCi} / \mathrm{L}$ \\
\hline & Total uranium & $1.0 \mathrm{U}$ & 3.9 & 2 & 1.5 & $\mu \mathrm{g} / \mathrm{L}$ \\
\hline \multirow[t]{4}{*}{$X 749-44 G$} & Gross alpha & $4 \mathrm{U}$ & $5 \mathrm{U}$ & $\mathrm{NA}$ & $\mathrm{NA}$ & $\mathrm{pCi} / \mathrm{L}$ \\
\hline & Gross beta & $9 \mathrm{U}$ & $11 \mathrm{U}$ & NA & NA & $\mathrm{pCi} / L$ \\
\hline & Technetium & $22 \mathrm{U}$ & $24 \mathrm{U}$ & NA & NA & $\mathrm{pCi} / \mathrm{L}$ \\
\hline & Total uranium & $1.0 \mathrm{U}$ & $1.1 \mathrm{~J}$ & NA & NA & $\mu \mathrm{g} / \mathrm{L}$ \\
\hline \multirow[t]{4}{*}{$X 749-45 G$} & Gross alpha & $6 U$ & $16 U$ & NA & NA & $\mathrm{pCi} / \mathrm{L}$ \\
\hline & Gross beta & $9 \mathrm{U}$ & $43 \mathrm{U}$ & NA & NA & $\mathrm{pCi} / \mathrm{L}$ \\
\hline & Technetium & $22 \mathrm{U}$ & $24 \mathrm{U}$ & $\mathrm{NA}$ & NA & $\mathrm{pCi} / \mathrm{L}$ \\
\hline & Total uranium & $1.0 \mathrm{U}$ & $1.5 \mathrm{~J}$ & NA & NA & $\mu \mathrm{g} / \mathrm{L}$ \\
\hline \multirow[t]{4}{*}{ X749-PZ02G } & Gross alpha & $3 \mathrm{U}$ & $5 \mathrm{U}$ & NA & NA & $\mathrm{pCi} / \mathrm{L}$ \\
\hline & Gross beta & $9 \mathrm{U}$ & $11 \mathrm{U}$ & NA & NA & $\mathrm{pCi} / \mathrm{L}$ \\
\hline & Technetium & $22 \mathrm{U}$ & 36 & NA & NA & $\mathrm{pCi} / \mathrm{L}$ \\
\hline & Total uranium & $1.0 \mathrm{U}$ & $1.0 \mathrm{U}$ & NA & NA & $\mu \mathrm{g} / \mathrm{L}$ \\
\hline
\end{tabular}


Table 4.4 (continued)

\begin{tabular}{|c|c|c|c|c|c|c|}
\hline Well & Parameter & $\operatorname{Min}^{\alpha}$ & $\operatorname{Max}^{a}$ & $\operatorname{Mean}^{b}$ & $\begin{array}{c}\text { Standard } \\
\text { deviation }{ }^{b}\end{array}$ & Units \\
\hline \multirow[t]{4}{*}{$\mathrm{X}-749-\mathrm{PZ03G}$} & Gross alpha & $2 U$ & $18 \mathrm{U}$ & $\mathrm{NA}$ & $\mathrm{NA}$ & $\overline{\mathrm{pCi} / \mathrm{L}}$ \\
\hline & Gross beta & $9 U$ & $38 \mathrm{U}$ & NA & NA & $\mathrm{pCi} / \mathrm{L}$ \\
\hline & Technetium & $22 \mathrm{U}$ & $24 U$ & NA & NA & $\mathrm{pCi} / \mathrm{L}$ \\
\hline & Total uranium & $1.0 \mathrm{U}$ & $1.0 \mathrm{U}$ & NA & NA & $\mu \mathrm{g} / \mathrm{L}$ \\
\hline \multirow[t]{4}{*}{ X749-PZ04G } & Gross alpha & $5 \mathrm{U}$ & $19 \mathrm{U}$ & NA & NA & $\mathrm{pCi} / \mathrm{L}$ \\
\hline & Gross beta & 15 & 84 & 59 & 31 & $\mathrm{pCi} / \mathrm{L}$ \\
\hline & Technetium & $22 \mathrm{U}$ & 135 & 87 & 53 & $\mathrm{pCi} / \mathrm{L}$ \\
\hline & Total uranium & $1.0 \mathrm{U}$ & $1.0 \mathrm{U}$ & NA & NA & $\mu \mathrm{g} / \mathrm{L}$ \\
\hline \multirow[t]{4}{*}{ X749-PZ05G } & Gross alpha & $19 \mathrm{U}$ & 474 & 152 & 222 & $\mathrm{pCi} / \mathrm{L}$ \\
\hline & Gross beta & $38 \mathrm{U}$ & 527 & 174 & 245 & $\mathrm{pCi} / \mathrm{L}$ \\
\hline & Technetium & $22 \mathrm{U}$ & $24 \mathrm{U}$ & NA & NA & $\mathrm{pCi} / \mathrm{L}$ \\
\hline & Total uranium & $3.4 \mathrm{U}$ & 80.2 & 40 & 39 & $\mu \mathrm{g} / \mathrm{L}$ \\
\hline \multicolumn{7}{|c|}{ Berea wells } \\
\hline \multirow[t]{4}{*}{ X749-14B } & Gross alpha & $4 \mathrm{U}$ & $5 \mathrm{U}$ & NA & NA & $\mathrm{pCi} / \mathrm{L}$ \\
\hline & Gross beta & $9 U$ & $11 \mathrm{U}$ & NA & NA & $\mathrm{pCi} / \mathrm{L}$ \\
\hline & Technetium & $22 \mathrm{U}$ & $24 U$ & NA & NA & $\mathrm{pCi} / \mathrm{L}$ \\
\hline & Total uranium & $1.0 \mathrm{U}$ & $1.0 \mathrm{U}$ & NA & NA & $\mu \mathrm{g} / \mathrm{L}$ \\
\hline \multirow[t]{4}{*}{ X749-50B } & Gross alpha & $13 \mathrm{U}$ & 24 & NA & NA & $\mathrm{pCi} / \mathrm{L}$ \\
\hline & Gross beta & 17 & 47 & 24 & 16 & $\mathrm{pCi} / \mathrm{L}$ \\
\hline & Technetium & $22 \mathrm{U}$ & $24 \mathrm{U}$ & NA & NA & $\mathrm{pCi} / \mathrm{L}$ \\
\hline & Total uranium & 1.5 & 4.9 & 3 & 1.6 & $\mu \mathrm{g} / \mathrm{L}$ \\
\hline \multirow[t]{4}{*}{ X749-51B } & Gross alpha & $6 \mathrm{U}$ & $10 \mathrm{U}$ & NA & $\mathrm{NA}$ & $\mathrm{pCi} / \mathrm{L}$ \\
\hline & Gross beta & $9 U$ & $19 U$ & NA & $\mathrm{NA}$ & $\mathrm{pCi} / \mathrm{L}$ \\
\hline & Technetium & $22 \mathrm{U}$ & $24 \mathrm{U}$ & NA & NA & $\mathrm{pCi} / \mathrm{L}$ \\
\hline & Total uranium & $1.0 \mathrm{U}$ & $1.0 \mathrm{U}$ & NA & NA & $\mu \mathrm{g} / \mathrm{L}$ \\
\hline \multirow[t]{4}{*}{ X749-54B } & Gross alpha & $5 \mathrm{U}$ & 94 & 45 & 38 & $\mathrm{pCi} / \mathrm{L}$ \\
\hline & Gross beta & $9 U$ & $41 \mathrm{U}$ & NA & NA & $\mathrm{pCi} / \mathrm{L}$ \\
\hline & Technetium & $22 U$ & $24 U$ & NA & NA & $\mathrm{pCi} / \mathrm{L}$ \\
\hline & Total uranium & $1.0 \mathrm{U}$ & $1.0 \mathrm{U}$ & NA & $\mathrm{NA}$ & $\mu \mathrm{g} / \mathrm{L}$ \\
\hline \multirow[t]{4}{*}{ X749-60B } & Gross alpha & 14 & 17 & 15 & 1.3 & $\mathrm{pCi} / \mathrm{L}$ \\
\hline & Gross beta & $10 \mathrm{U}$ & 13 & NA & NA & $\mathrm{pCi} / \mathrm{L}$ \\
\hline & Technetium & $22 \mathrm{U}$ & $24 \mathrm{U}$ & NA & NA & $\mathrm{pCi} / \mathrm{L}$ \\
\hline & Total uranium & 2.4 & 3.1 & 3 & 0.3 & $\mu \mathrm{g} / \mathrm{L}$ \\
\hline \multirow[t]{4}{*}{$X 749-64 B$} & Gross alpha & $14 \mathrm{U}$ & 81 & NA & NA & $\mathrm{pCi} / \mathrm{L}$ \\
\hline & Gross beta & 25 & 89 & NA & NA & $\mathrm{pCi} / \mathrm{L}$ \\
\hline & Technetium & $22 \mathrm{U}$ & $32 \mathrm{U}$ & NA & NA & $\mathrm{pCi} / \mathrm{L}$ \\
\hline & Total uranium & 1.3 & 4 & 2 & 1.2 & $\mu \mathrm{g} / \mathrm{L}$ \\
\hline
\end{tabular}

${ }^{a}$ Qualifiers include: U-parameter was not detected; the result is the attainable detection limit for the sample. J-result must be considered an estimate.

WA-at least $50 \%$ of the results for the given parameter at this location were below detection; therefore, mean and standard deviation functions are not applicable. 
Table 4.5. Portsmouth groundwater monitoring for 1994:

results for volatile organic compounds at the X-231B oil biodegratation plot

\begin{tabular}{|c|c|c|c|c|c|c|}
\hline Well & Parameter ${ }^{a}$ & $\operatorname{Min}^{b}$ & $\operatorname{Max}^{b}$ & Mean $^{c}$ & $\begin{array}{c}\text { Standard } \\
\text { deviation }^{c}\end{array}$ & Units \\
\hline \multicolumn{7}{|c|}{ Gallia wells } \\
\hline \multirow[t]{3}{*}{$\mathrm{X} 231 \mathrm{~B}-02 \mathrm{G}$} & $1,1,1-\mathrm{TCA}$ & $50 \mathrm{U}$ & $100 \mathrm{U}$ & $\mathrm{NA}$ & NA & $\mu \mathrm{g} / \mathrm{L}$ \\
\hline & 1,1-DCE & 43 & $100 \mathrm{U}$ & NA & NA & $\mu \mathrm{g} / \mathrm{L}$ \\
\hline & TCE & 1510 & 2640 & 1928 & 501 & $\mu \mathrm{g} / \mathrm{L}$ \\
\hline \multirow[t]{3}{*}{$\mathrm{X} 231 \mathrm{~B}-03 \mathrm{G}$} & $1,1,1-\mathrm{TCA}$ & 54 & 210 & 134 & 67 & $\mu \mathrm{g} / \mathrm{L}$ \\
\hline & 1,1-DCE & 41 & 170 & 102 & 56 & $\mu \mathrm{g} / \mathrm{L}$ \\
\hline & TCE & 610 & 1110 & 800 & 228 & $\mu \mathrm{g} / \mathrm{L}$ \\
\hline \multirow[t]{3}{*}{ X231B-04G } & $1,1,1-\mathrm{TCA}$ & $20 \mathrm{U}$ & $20 \mathrm{U}$ & NA & NA & $\mu \mathrm{g} / \mathrm{L}$ \\
\hline & 1,1-DCE & $20 U$ & $20 \mathrm{U}$ & NA & NA & $\mu \mathrm{g} / \mathrm{L}$ \\
\hline & TCE & 218 & 305 & 281 & 42 & $\mu \mathrm{g} / \mathrm{L}$ \\
\hline \multirow[t]{3}{*}{$\mathrm{X} 231 \mathrm{~B}-06 \mathrm{G}$} & $1,1,1-\mathrm{TCA}$ & 300 & 520 & 405 & 110 & $\mu \mathrm{g} / \mathrm{L}$ \\
\hline & 1,1-DCE & 200 & 279 & 227 & 45 & $\mu \mathrm{g} / \mathrm{L}$ \\
\hline & TCE & 61 & 200 & 116 & 74 & $\mu \mathrm{g} / \mathrm{L}$ \\
\hline \multirow[t]{3}{*}{ X231B-14G } & $1,1,1-\mathrm{TCA}$ & $2 U$ & $2 \mathrm{U}$ & $\mathrm{NA}$ & NA & $\mu \mathrm{g} / \mathrm{L}$ \\
\hline & 1,1-DCE & $2 \mathrm{U}$ & $2 \mathrm{U}$ & NA & NA & $\mu \mathrm{g} / \mathrm{L}$ \\
\hline & TCE & 39 & 52 & 43 & 6 & $\mu \mathrm{g} / \mathrm{L}$ \\
\hline \multirow[t]{3}{*}{$\mathrm{X} 231 \mathrm{~B}-15 \mathrm{G}$} & $1,1,1-\mathrm{TCA}$ & $2 \mathrm{U}$ & 6 & NA & NA & $\mu \mathrm{g} / \mathrm{L}$ \\
\hline & 1,1-DCE & $2 \mathrm{U}$ & 4 & NA & NA & $\mu \mathrm{g} / \mathrm{L}$ \\
\hline & TCE & 41 & 58 & 48 & 7 & $\mu \mathrm{g} / \mathrm{L}$ \\
\hline \multirow[t]{3}{*}{$\mathrm{X} 231 \mathrm{~B}-16 \mathrm{G}$} & $1,1,1-\mathrm{TCA}$ & $2 \mathrm{U}$ & $2 \mathrm{U}$ & NA & NA & $\mu \mathrm{g} / \mathrm{L}$ \\
\hline & 1,1-DCE & $2 \mathrm{U}$ & $2 \mathrm{U}$ & NA & NA & $\mu \mathrm{g} / \mathrm{L}$ \\
\hline & TCE & $2 \mathrm{U}$ & $2 U$ & NA & NA & $\mu \mathrm{g} / \mathrm{L}$ \\
\hline \multirow[t]{3}{*}{ X231B-23G } & $1,1,1-\mathrm{TCA}$ & 2 & $20 \mathrm{U}$ & NA & NA & $\mu \mathrm{g} / \mathrm{L}$ \\
\hline & 1,1-DCE & $2 U$ & $20 \mathrm{U}$ & NA & NA & $\mu \mathrm{g} / \mathrm{L}$ \\
\hline & TCE & 13 & 26 & 16 & 7 & $\mu \mathrm{g} / \mathrm{L}$ \\
\hline \multirow[t]{3}{*}{$\mathrm{X} 231 \mathrm{~B}-27 \mathrm{G}$} & $1,1,1-\mathrm{TCA}$ & $2 U$ & $2 \mathrm{U}$ & $\mathrm{NA}$ & NA & $\mu \mathrm{g} / \mathrm{L}$ \\
\hline & 1,1-DCE & $2 U$ & $2 U$ & NA & NA & $\mu \mathrm{g} / \mathrm{L}$ \\
\hline & TCE & $2 U$ & $2 \mathrm{U}$ & NA & NA & $\mu \mathrm{g} / \mathrm{L}$ \\
\hline \multirow[t]{3}{*}{$\mathrm{X} 231 \mathrm{~B}-28 \mathrm{G}$} & $1,1,1-\mathrm{TCA}$ & $2 \mathrm{U}$ & $2 \mathrm{U}$ & NA & NA & $\mu \mathrm{g} / \mathrm{L}$ \\
\hline & 1,1-DCE & $2 \mathrm{U}$ & $2 \mathrm{U}$ & NA & $\mathrm{NA}$ & $\mu \mathrm{g} / \mathrm{L}$ \\
\hline & TCE & $2 U$ & $2 U$ & NA & NA & $\mu \mathrm{g} / \mathrm{L}$ \\
\hline \multirow[t]{3}{*}{$\mathrm{X} 231 \mathrm{~B}-37 \mathrm{G}$} & $1,1,1-\mathrm{TCA}$ & $2 \mathrm{U}$ & $2 \mathrm{U}$ & $\mathrm{NA}$ & NA & $\mu \mathrm{g} / \mathrm{L}$ \\
\hline & 1,1-DCE & 7 & 8 & 7.5 & 0.6 & $\mu \mathrm{g} / \mathrm{L}$ \\
\hline & TCE & 62 & 74 & 69 & 5 & $\mu \mathrm{g} / \mathrm{L}$ \\
\hline
\end{tabular}


Table 4.5 (continued)

\begin{tabular}{|c|c|c|c|c|c|c|}
\hline Well & Parameter ${ }^{a}$ & $\operatorname{Min}^{b}$ & $\operatorname{Max}^{b}$ & Mean $^{c}$ & $\begin{array}{c}\text { Standard } \\
\text { deviation }^{c}\end{array}$ & Units \\
\hline \multicolumn{7}{|c|}{ Berea wells } \\
\hline \multirow[t]{3}{*}{$\mathrm{X} 231 \mathrm{~B}-24 \mathrm{~B}$} & $1,1,1-\mathrm{TCA}$ & $2 \mathrm{U}$ & $2 \mathrm{U}$ & NA & NA & $\mu \mathrm{g} / \mathrm{L}$ \\
\hline & 1,1-DCE & $2 \mathrm{U}$ & $2 \mathrm{U}$ & NA & NA & $\mu \mathrm{g} / \mathrm{L}$ \\
\hline & TCE & $2 U$ & $2 \mathrm{U}$ & NA & NA & $\mu \mathrm{g} / \mathrm{L}$ \\
\hline \multirow[t]{3}{*}{$\mathrm{X} 231 \mathrm{~B}-32 \mathrm{~B}$} & $1,1,1-\mathrm{TCA}$ & $2 U$ & $2 \mathrm{U}$ & NA & NA & $\mu \mathrm{g} / \mathrm{L}$ \\
\hline & 1,1-DCE & $2 U$ & $2 U$ & NA & NA & $\mu \mathrm{g} / \mathrm{L}$ \\
\hline & TCE & $2 U$ & $2 \mathrm{U}$ & NA & NA & $\mu \mathrm{g} / \mathrm{L}$ \\
\hline \multirow[t]{3}{*}{$\mathrm{X} 231 \mathrm{~B}-33 \mathrm{~B}$} & $1,1,1-\mathrm{TCA}$ & $2 U$ & $2 U$ & NA & NA & $\mu \mathrm{g} / \mathrm{L}$ \\
\hline & 1,1-DCE & $2 \mathrm{U}$ & $2 U$ & NA & NA & $\mu \mathrm{g} / \mathrm{L}$ \\
\hline & TCE & $2 U$ & $2 \mathrm{U}$ & NA & NA & $\mu \mathrm{g} / \mathrm{L}$ \\
\hline \multirow[t]{3}{*}{$\mathrm{X} 231 \mathrm{~B}-34 \mathrm{~B}$} & $1,1,1-\mathrm{TCA}$ & $2 \mathrm{U}$ & $2 U$ & NA & NA & $\mu \mathrm{g} / \mathrm{L}$ \\
\hline & 1,1-DCE & $2 U$ & $2 U$ & NA & NA & $\mu \mathrm{g} / \mathrm{L}$ \\
\hline & TCE & $2 U$ & $2 \mathrm{U}$ & NA & NA & $\mu \mathrm{g} / \mathrm{L}$ \\
\hline
\end{tabular}

${ }^{a}$ Parameter abbreviations: $1,1,1-\mathrm{TCA}=1,1,1$-trichloroethane; $1,1-\mathrm{DCE}=1,1$-dichloroethene; and TCE = trichloroethylene.

${ }^{b}$ Qualifiers include: $\mathrm{U}$-parameter was not detected; the result is the attainable detection limit for the sample.

NA - at least $50 \%$ of the results for the given parameter at this location were below detection; therefore, mean and standard deviation functions are not applicable. 
Table 4.6. Portsmouth groundwater monitoring for 1994: results for radionuclide parameters at $\mathrm{X}-231 \mathrm{~B}$

\begin{tabular}{|c|c|c|c|c|c|c|}
\hline Well & Parameter & $\operatorname{Min}^{a}$ & $\operatorname{Max}^{a}$ & $\operatorname{Mean}^{b}$ & $\begin{array}{c}\text { Standard } \\
\text { deviation }^{b}\end{array}$ & Units \\
\hline \multicolumn{7}{|c|}{ Gallia wells } \\
\hline \multirow[t]{4}{*}{$\mathrm{X} 231 \mathrm{~B}-02 \mathrm{G}$} & Gross alpha & $5 \mathrm{U}$ & $19 \mathrm{U}$ & NA & NA & $\mathrm{pCi} / \mathrm{L}$ \\
\hline & Gross beta & $11 \mathrm{U}$ & $41 \mathrm{U}$ & NA & NA & $\mathrm{pCi} / \mathrm{L}$ \\
\hline & Technetium & $22 \mathrm{U}$ & $24 \mathrm{U}$ & NA & NA & $\mathrm{pCi} / \mathrm{L}$ \\
\hline & Total uranium & $1.0 \mathrm{U}$ & 1.1 & NA & NA & $\mu \mathrm{g} / \mathrm{L}$ \\
\hline \multirow[t]{4}{*}{$\mathrm{X} 231 \mathrm{~B}-03 \mathrm{G}$} & Gross alpha & $4 \mathrm{U}$ & $17 \mathrm{U}$ & NA & NA & $\mathrm{pCi} / \mathrm{L}$ \\
\hline & Gross beta & 10 & $43 \mathrm{U}$ & NA & NA & $\mathrm{pCi} / \mathrm{L}$ \\
\hline & Technetium & $22 \mathrm{U}$ & 31 & NA & NA & $\mathrm{pCi} / \mathrm{L}$ \\
\hline & Total uranium & $1.0 \mathrm{U}$ & $2.9 \mathrm{U}$ & NA & NA & $\mu \mathrm{g} / \mathrm{L}$ \\
\hline \multirow[t]{4}{*}{$\mathrm{X} 231 \mathrm{~B}-04 \mathrm{G}$} & Gross alpha & $7 \mathrm{U}$ & 64 & 29 & 25 & $\mathrm{pCi} / \mathrm{L}$ \\
\hline & Gross beta & $11 \mathrm{U}$ & $43 \mathrm{U}$ & NA & NA & $\mathrm{pCi} / \mathrm{L}$ \\
\hline & Technetium & $22 \mathrm{U}$ & 23 & NA & NA & $\mathrm{pCi} / \mathrm{L}$ \\
\hline & Total uranium & 2.5 & 18.9 & 8.1 & 7.4 & $\mu \mathrm{g} / \mathrm{L}$ \\
\hline \multirow[t]{4}{*}{$\mathrm{X} 231 \mathrm{~B}-06 \mathrm{G}$} & Gross alpha & 14 & 96 & 40 & 40 & $\mathrm{pCi} / \mathrm{L}$ \\
\hline & Gross beta & 304 & 824 & 548 & 242 & $\mathrm{pCi} / \mathrm{L}$ \\
\hline & Technetium & 359 & 1041 & 715 & 324 & $\mathrm{pCi} / \mathrm{L}$ \\
\hline & Total uranium & 2.7 & 17.1 & 9.2 & 6.0 & $\mu \mathrm{g} / \mathrm{L}$ \\
\hline \multirow[t]{4}{*}{$\mathrm{X} 231 \mathrm{~B}-14 \mathrm{G}$} & Gross alpha & $4 U$ & $5 \mathrm{U}$ & NA & NA & $\mathrm{pCi} / \mathrm{L}$ \\
\hline & Gross beta & $9 U$ & $11 \mathrm{U}$ & NA & NA & $\mathrm{pCi} / \mathrm{L}$ \\
\hline & Technetium & $22 U$ & 27 & NA & NA & $\mathrm{pCi} / \mathrm{L}$ \\
\hline & Total uranium & $1.0 \mathrm{U}$ & $1.0 \mathrm{U}$ & NA & NA & $\mu \mathrm{g} / \mathrm{L}$ \\
\hline \multirow[t]{4}{*}{$\mathrm{X} 231 \mathrm{~B}-15 \mathrm{G}$} & Gross alpha & $10 \mathrm{U}$ & $11 \mathrm{U}$ & NA & NA & $\mathrm{pCi} / \mathrm{L}$ \\
\hline & Gross beta & $10 \mathrm{U}$ & $21 \mathrm{U}$ & NA & NA & $\mathrm{pCi} / \mathrm{L}$ \\
\hline & Technetium & $22 \mathrm{U}$ & $22 \mathrm{U}$ & NA & NA & $\mathrm{pCi} / \mathrm{L}$ \\
\hline & Total uranium & $1.0 \mathrm{U}$ & $1.0 \mathrm{U}$ & NA & NA & $\mu \mathrm{g} / \mathrm{L}$ \\
\hline \multirow[t]{4}{*}{$\mathrm{X} 231 \mathrm{~B}-16 \mathrm{G}$} & Gross alpha & $14 \mathrm{U}$ & $18 \mathrm{U}$ & NA & NA & $\mathrm{pCi} / \mathrm{L}$ \\
\hline & Gross beta & 11 & $22 \mathrm{U}$ & NA & NA & $\mathrm{pCi} / \mathrm{L}$ \\
\hline & Technetium & $22 \mathrm{U}$ & $22 \mathrm{U}$ & NA & NA & $\mathrm{pCi} / \mathrm{L}$ \\
\hline & Total uranium & $1.0 \mathrm{U}$ & $1.0 \mathrm{U}$ & NA & NA & $\mu \mathrm{g} / \mathrm{L}$ \\
\hline \multirow[t]{4}{*}{$\mathrm{X} 231 \mathrm{~B}-23 \mathrm{G}$} & Gross alpha & $8 \mathrm{U}$ & $18 \mathrm{U}$ & NA & NA & $\mathrm{pCi} / \mathrm{L}$ \\
\hline & Gross beta & $10 \mathrm{U}$ & $43 \mathrm{U}$ & NA & NA & $\mathrm{pCi} / \mathrm{L}$ \\
\hline & Technetium & $22 \mathrm{U}$ & $22 \mathrm{U}$ & NA & NA & $\mathrm{pCi} / \mathrm{L}$ \\
\hline & Total uranium & $1.0 \mathrm{U}$ & 1.0 & NA & NA & $\mu \mathrm{g} / \mathrm{L}$ \\
\hline \multirow[t]{4}{*}{$\mathrm{X} 231 \mathrm{~B}-27 \mathrm{G}$} & Gross alpha & $5 \mathrm{U}$ & $15 \mathrm{U}$ & NA & NA & $\mathrm{pCi} / \mathrm{L}$ \\
\hline & Gross beta & $9 \mathrm{U}$ & $43 \mathrm{U}$ & NA & NA & $\mathrm{pCi} / \mathrm{L}$ \\
\hline & Technetium & $22 U$ & $22 \mathrm{U}$ & $\mathrm{NA}$ & NA & $\mathrm{pCi} / \mathrm{L}$ \\
\hline & Total uranium & $1.0 \mathrm{U}$ & 1.1 & NA & $\mathrm{NA}$ & $\mu \mathrm{g} / \mathrm{L}$ \\
\hline
\end{tabular}


Table 4.6 (continued)

\begin{tabular}{|c|c|c|c|c|c|c|}
\hline Well & Parameter & $\operatorname{Min}^{\alpha}$ & $\operatorname{Max}^{a}$ & Mean $^{b}$ & $\begin{array}{c}\text { Standard } \\
\text { deviation }^{b}\end{array}$ & Units \\
\hline \multirow[t]{4}{*}{$\mathrm{X} 231 \mathrm{~B}-28 \mathrm{G}$} & Gross alpha & $9 \mathrm{U}$ & $11 \mathrm{U}$ & NA & NA & $\mathrm{pCi} / \mathrm{L}$ \\
\hline & Gross beta & $10 \mathrm{U}$ & $21 \mathrm{U}$ & NA & NA & $\mathrm{pCi} / \mathrm{L}$ \\
\hline & Technetium & $22 \mathrm{U}$ & $22 \mathrm{U}$ & NA & NA & $\mathrm{pCi} / \mathrm{L}$ \\
\hline & Total uranium & $1.0 \mathrm{U}$ & $1.0 \mathrm{U}$ & NA & NA & $\mu \mathrm{g} / \mathrm{L}$ \\
\hline \multirow[t]{4}{*}{$\mathrm{X} 231 \mathrm{~B}-37 \mathrm{G}$} & Gross alpha & $5 \mathrm{U}$ & $9 \mathrm{U}$ & NA & NA & $\mathrm{pCi} / \mathrm{L}$ \\
\hline & Gross beta & $9 \mathrm{U}$ & $21 \mathrm{U}$ & NA & NA & $\mathrm{pCi} / \mathrm{L}$ \\
\hline & Technetium & $22 \mathrm{U}$ & 36 & NA & NA & $\mathrm{pCi} / \mathrm{L}$ \\
\hline & Total uranium & $1.0 \mathrm{U}$ & $1.0 \mathrm{U}$ & NA & NA & $\mu \mathrm{g} / \mathrm{L}$ \\
\hline \multicolumn{7}{|c|}{ Berea wells } \\
\hline \multirow[t]{4}{*}{$\mathrm{X} 231 \mathrm{~B}-24 \mathrm{~B}$} & Gross apha & $14 \mathrm{U}$ & $21 \mathrm{U}$ & NA & NA & $\mathrm{pCi} / \mathrm{L}$ \\
\hline & Gross beta & 12 & $22 \mathrm{U}$ & NA & NA & $\mathrm{pCi} / \mathrm{L}$ \\
\hline & Technetium & $22 \mathrm{U}$ & $22 \mathrm{U}$ & NA & NA & $\mathrm{pCi} / \mathrm{L}$ \\
\hline & Total uranium & $1.0 \mathrm{U}$ & 1.8 & 1.4 & 0.6 & $\mu \mathrm{g} / \mathrm{L}$ \\
\hline \multirow[t]{4}{*}{$\mathrm{X} 231 \mathrm{~B}-32 \mathrm{~B}$} & Gross alpha & $17 \mathrm{U}$ & 25 & NA & NA & $\mathrm{pCi} / \mathrm{L}$ \\
\hline & Gross beta & $19 U$ & 27 & NA & NA & $\mathrm{pCi} / \mathrm{L}$ \\
\hline & Technetium & $22 \mathrm{U}$ & $22 \mathrm{U}$ & NA & NA & $\mathrm{pCi} / \mathrm{L}$ \\
\hline & Total uranium & $1.0 \mathrm{U}$ & 1.2 & NA & NA & $\mu \mathrm{g} / \mathrm{L}$ \\
\hline \multirow[t]{4}{*}{$\mathrm{X} 231 \mathrm{~B}-33 \mathrm{~B}$} & Gross alpha & $6 \mathrm{U}$ & 15 & NA & NA & $\mathrm{pCi} / \mathrm{L}$ \\
\hline & Gross beta & $10 \mathrm{U}$ & 20 & NA & NA & $\mathrm{pCi} / \mathrm{L}$ \\
\hline & Technetium & $22 \mathrm{U}$ & $24 \mathrm{U}$ & NA & NA & $\mathrm{pCi} / \mathrm{L}$ \\
\hline & Total uranium & $1.0 \mathrm{U}$ & $1.0 \mathrm{U}$ & NA & NA & $\mu \mathrm{g} / \mathrm{L}$ \\
\hline \multirow[t]{4}{*}{$\mathrm{X} 231 \mathrm{~B}-34 \mathrm{~B}$} & Gross alpha & 14 & 26 & 19 & 5.3 & $\mathrm{pCi} / \mathrm{L}$ \\
\hline & Gross beta & 10 & 15 & NA & NA & $\mathrm{pCi} / \mathrm{L}$ \\
\hline & Technetium & $22 \mathrm{U}$ & $22 \mathrm{U}$ & NA & NA & $\mathrm{pCi} / \mathrm{L}$ \\
\hline & Total uranium & 1.6 & 2 & 1.8 & 0.2 & $\mu \mathrm{g} / \mathrm{L}$ \\
\hline
\end{tabular}

${ }^{a}$ Qualifiers include: U-parameter was not detected; the result is the attainable detection limit for the sample.

WA-at least $50 \%$ of the results for the given parameter at this location were below detection; therefore, mean and standard deviation functions are not applicable. 
Table 4.7. Portsmouth groundwater monitoring for 1994: results for volatile organic compounds at $X-616$

\begin{tabular}{|c|c|c|c|c|c|c|}
\hline Well & Parameter & $\operatorname{Min}^{a}$ & $\operatorname{Max}^{a}$ & $\operatorname{Mean}^{b}$ & $\begin{array}{c}\text { Standard } \\
\text { deviation }^{b}\end{array}$ & Units \\
\hline \multicolumn{7}{|c|}{ Gallia wells } \\
\hline \multirow[t]{2}{*}{ X616-05G } & 1,1,1-Trichloroethane & $2 U$ & $5 \mathrm{U}$ & NA & NA & $\mu \mathrm{g} / \mathrm{L}$ \\
\hline & Trichloroethylene & $2 \mathrm{U}$ & $5 \mathrm{U}$ & NA & NA & $\mu \mathrm{g} / \mathrm{L}$ \\
\hline \multirow[t]{2}{*}{$\mathrm{X} 616-09 \mathrm{G}$} & 1,1,1-Trichloroethane & $2 U$ & $5 \mathrm{U}$ & NA & NA & $\mu \mathrm{g} / \mathrm{L}$ \\
\hline & Trichloroethylene & 3 & 4 & 3.3 & 0.6 & $\mu \mathrm{g} / \mathrm{L}$ \\
\hline \multirow[t]{2}{*}{$X 616-10 G$} & 1,1,1-Trichloroethane & $2 U$ & $5 \mathrm{U}$ & NA & NA & $\mu \mathrm{g} / \mathrm{L}$ \\
\hline & Trichloroethylene & $2 U$ & $5 U$ & $\mathrm{NA}$ & NA & $\mu g / L$ \\
\hline \multirow[t]{2}{*}{ X616-13G } & 1,1,1-Trichloroethane & $2 \mathrm{U}$ & $2 \mathrm{U}$ & NA & NA & $\mu \mathrm{g} / \mathrm{L}$ \\
\hline & Trichloroethylene & $2 U$ & $2 \mathrm{U}$ & NA & NA & $\mu \mathrm{g} / \mathrm{L}$ \\
\hline \multirow[t]{2}{*}{$\mathrm{X} 616-14 \mathrm{G}$} & 1,1,1-Trichloroethane & $2 \mathrm{U}$ & $5 \mathrm{U}$ & NA & NA & $\mu \mathrm{g} / \mathrm{L}$ \\
\hline & Trichloroethylene & $2 U$ & $5 \mathrm{U}$ & NA & NA & $\mu \mathrm{g} / \mathrm{L}$ \\
\hline \multirow[t]{2}{*}{$\mathrm{X} 616-16 \mathrm{G}$} & 1,1,1-Trichloroethane & $2 \mathrm{U}$ & $5 \mathrm{U}$ & NA & NA & $\mu \mathrm{g} / \mathrm{L}$ \\
\hline & Trichloroethylene & 4 & 5 & 4.7 & 0.6 & $\mu \mathrm{g} / \mathrm{L}$ \\
\hline \multirow[t]{2}{*}{$\mathrm{X} 616-17 \mathrm{G}$} & 1,1,1-Trichloroethane & $2 \mathrm{U}$ & $5 \mathrm{U}$ & NA & NA & $\mu g / L$ \\
\hline & Trichloroethylene & $2 U$ & $5 U$ & NA & NA & $\mu \mathrm{g} / \mathrm{L}$ \\
\hline \multirow[t]{2}{*}{$\mathrm{X616-21G}$} & 1,1,1-Trichloroethane & $2 U$ & $5 \mathrm{U}$ & NA & NA & $\mu \mathrm{g} / \mathrm{L}$ \\
\hline & Trichloroethylene & $2 U$ & $5 \mathrm{U}$ & NA & NA & $\mu \mathrm{g} / \mathrm{L}$ \\
\hline \multirow[t]{2}{*}{$\mathrm{X} 616-22 \mathrm{G}$} & 1,1,1-Trichloroethane & $2 U$ & $5 \mathrm{U}$ & NA & NA & $\mu \mathrm{g} / \mathrm{L}$ \\
\hline & Trichloroethylene & $2 U$ & $5 \mathrm{U}$ & NA & NA & $\mu \mathrm{g} / \mathrm{L}$ \\
\hline \multirow[t]{2}{*}{$\mathrm{X} 616-25 \mathrm{G}$} & 1,1,1-Trichloroethane & $2 \mathrm{U}$ & $5 \mathrm{U}$ & NA & NA & $\mu \mathrm{g} / \mathrm{L}$ \\
\hline & Trichloroethylene & $2 U$ & $5 \mathrm{U}$ & NA & NA & $\mu \mathrm{g} / \mathrm{L}$ \\
\hline \multirow[t]{2}{*}{$\mathrm{X} 616-26 \mathrm{G}$} & 1,1,1-Trichloroethane & $2 U$ & $5 \mathrm{U}$ & NA & NA & $\mu \mathrm{g} / \mathrm{L}$ \\
\hline & Trichloroethylene & $2 \mathrm{U}$ & $5 \mathrm{U}$ & NA & NA & $\mu \mathrm{g} / \mathrm{L}$ \\
\hline \multicolumn{7}{|c|}{ Berea wells } \\
\hline \multirow[t]{2}{*}{ X616-19B } & 1,1,1-Trichloroethane & $2 \mathrm{U}$ & $5 \mathrm{U}$ & NA & NA & $\mu \mathrm{g} / \mathrm{L}$ \\
\hline & Trichloroethylene & $2 \mathrm{U}$ & 3 & NA & NA & $\mu \mathrm{g} / \mathrm{L}$ \\
\hline \multirow[t]{2}{*}{$\mathrm{X} 616-20 \mathrm{~B}$} & 1,1,1-Trichloroethane & $2 U$ & $5 \mathrm{U}$ & NA & NA & $\mu \mathrm{g} / \mathrm{L}$ \\
\hline & Trichloroethylene & 15 & 21 & 18.0 & 3.0 & $\mu \mathrm{g} / \mathrm{L}$ \\
\hline \multirow[t]{2}{*}{$\mathrm{X} 616-24 \mathrm{~B}$} & 1,1,1-Trichloroethane & $2 \mathrm{U}$ & $5 \mathrm{U}$ & NA & NA & $\mu g / L$ \\
\hline & Trichloroethylene & $2 \mathrm{U}$ & $5 \mathrm{U}$ & NA & NA & $\mu \mathrm{g} / \mathrm{L}$ \\
\hline \multirow[t]{2}{*}{ X616-28B } & 1,1,1-Trichloroethane & $2 \mathrm{U}$ & 4 & NA & NA & $\mu \mathrm{g} / \mathrm{L}$ \\
\hline & Trichloroethylene & $2 \mathrm{U}$ & $5 \mathrm{U}$ & NA & NA & $\mu \mathrm{g} / \mathrm{L}$ \\
\hline
\end{tabular}

${ }^{a}$ Qualifiers include: U-parameter was not detected; the result is the attainable detection limit for the sample.

${ }^{b} \mathrm{NA}$-at least $50 \%$ of the results for the given parameter at this location were below detection; therefore, mean and standard deviation functions are not applicable. 
Table 4.8. Portsmouth groundwater monitoring for 1994: dissolved and total chromium results for $X-616$

\begin{tabular}{|c|c|c|c|c|c|c|}
\hline Well & Parameter & $\operatorname{Min}^{a}$ & $\operatorname{Max}^{a}$ & $\operatorname{Mean}^{b}$ & $\begin{array}{c}\text { Standard } \\
\text { deviation }^{b}\end{array}$ & Units \\
\hline \multicolumn{7}{|c|}{ Gallia wells } \\
\hline \multirow[t]{2}{*}{$\mathrm{X} 616-05 \mathrm{G}$} & Dissolved $\mathrm{Cr}^{\varepsilon}$ & 339 & 465 & 402 & 89 & $\mu \mathrm{g} / \mathrm{L}$ \\
\hline & Total $\mathrm{Cr}$ & 464 & 1520 & 1104 & 563 & $\mu \mathrm{g} / \mathrm{L}$ \\
\hline \multirow[t]{2}{*}{$\mathrm{X} 616-09 \mathrm{G}$} & Dissolved Cr & $2.5 \mathrm{U}$ & 6.2 & NA & NA & $\mu \mathrm{g} / \mathrm{L}$ \\
\hline & Total $\mathrm{Cr}$ & $2.5 \mathrm{U}$ & $4.3 \mathrm{U}$ & NA & NA & $\mu \mathrm{g} / \mathrm{L}$ \\
\hline \multirow[t]{2}{*}{ X616-10G } & Dissolved Cr & $2.5 \mathrm{U}$ & $2.6 \mathrm{U}$ & NA & NA & $\mu \mathrm{g} / \mathrm{L}$ \\
\hline & Total $\mathrm{Cr}$ & $2.5 \mathrm{U}$ & $2.6 \mathrm{U}$ & NA & NA & $\mu \mathrm{g} / \mathrm{L}$ \\
\hline \multirow[t]{2}{*}{$X 616-13 G$} & Dissolved $\mathrm{Cr}$ & $4.3 \mathrm{U}$ & $12.5 \mathrm{U}$ & NA & NA & $\mu \mathrm{g} / \mathrm{L}$ \\
\hline & Total $\mathrm{Cr}$ & $5.1 \mathrm{U}$ & 189 & NA & NA & $\mu \mathrm{g} / \mathrm{L}$ \\
\hline \multirow[t]{2}{*}{$\mathrm{X} 616-14 \mathrm{G}$} & Dissolved $\mathrm{Cr}$ & $12.5 \mathrm{U}$ & 8.2 & NA & NA & $\mu \mathrm{g} / \mathrm{L}$ \\
\hline & Total $\mathrm{Cr}$ & $2.5 \mathrm{U}$ & 9.5 & NA & NA & $\mu \mathrm{g} / \mathrm{L}$ \\
\hline \multirow[t]{2}{*}{$\mathrm{X} 616-16 \mathrm{G}$} & Dissolved $\mathrm{Cr}$ & $2.6 \mathrm{U}$ & 3.2 & NA & NA & $\mu \mathrm{g} / \mathrm{L}$ \\
\hline & Total $\mathrm{Cr}$ & $2.5 \mathrm{U}$ & $2.6 \mathrm{U}$ & NA & NA & $\mu \mathrm{g} / \mathrm{L}$ \\
\hline \multirow[t]{2}{*}{$\mathrm{X} 616-17 \mathrm{G}$} & Dissolved $\mathrm{Cr}$ & $2.5 \mathrm{U}$ & $4.3 \mathrm{U}$ & NA & NA & $\mu \mathrm{g} / \mathrm{L}$ \\
\hline & Total $\mathbf{C r}$ & 21.1 & 52.9 & 40.2 & 16.9 & $\mu \mathrm{g} / \mathrm{L}$ \\
\hline \multirow[t]{2}{*}{$\mathrm{X} 616-21 \mathrm{G}$} & Dissolved $\mathrm{Cr}$ & $2.5 \mathrm{U}$ & $4.3 \mathrm{U}$ & NA & NA & $\mu \mathrm{g} / \mathrm{L}$ \\
\hline & Total $\mathrm{Cr}$ & 5.3 & 14.2 & 8.4 & 5.1 & $\mu \mathrm{g} / \mathrm{L}$ \\
\hline \multirow[t]{2}{*}{$\mathrm{X} 616-22 \mathrm{G}$} & Dissolved $\mathrm{Cr}$ & $2.5 \mathrm{U}$ & $4.3 \mathrm{U}$ & $\mathrm{NA}$ & NA & $\mu \mathrm{g} / \mathrm{L}$ \\
\hline & Total $\mathrm{Cr}$ & $4.3 \mathrm{U}$ & 17.2 & 7.9 & 8.2 & $\mu \mathrm{g} / \mathrm{L}$ \\
\hline \multirow[t]{2}{*}{$\mathrm{X} 616-25 \mathrm{G}$} & Dissolved $\mathrm{Cr}$ & $2.5 \mathrm{U}$ & $4.3 \mathrm{U}$ & NA & NA & $\mu \mathrm{g} / \mathrm{L}$ \\
\hline & Total $\mathrm{Cr}$ & $4.3 \mathrm{U}$ & 21.4 & 13.9 & 10.3 & $\mu \mathrm{g} / \mathrm{L}$ \\
\hline \multirow[t]{2}{*}{$\mathrm{X} 616-26 \mathrm{G}$} & Dissolved $\mathrm{Cr}$ & $2.6 \mathrm{U}$ & $12.5 \mathrm{U}$ & NA & NA & $\mu \mathrm{g} / \mathrm{L}$ \\
\hline & Total $\mathrm{Cr}$ & $2.5 \mathrm{U}$ & 2.8 & NA & NA & $\mu \mathrm{g} / \mathrm{L}$ \\
\hline \multicolumn{7}{|c|}{ Berea wells } \\
\hline \multirow[t]{2}{*}{$\mathrm{X} 616-19 B$} & Dissolved $\mathrm{Cr}$ & $2.5 \mathrm{U}$ & $2.6 \mathrm{U}$ & NA & NA & $\mu \mathrm{g} / \mathrm{L}$ \\
\hline & Total $\mathrm{Cr}$ & $2.5 \mathrm{U}$ & 4.2 & NA & NA & $\mu \mathrm{g} / \mathrm{L}$ \\
\hline \multirow[t]{2}{*}{$\mathrm{X} 616-20 \mathrm{~B}$} & Dissolved $\mathrm{Cr}$ & $2.5 \mathrm{U}$ & $4.3 \mathrm{U}$ & NA & NA & $\mu \mathfrak{g} / \mathrm{L}$ \\
\hline & Total $\mathrm{Cr}$ & $4.3 \mathrm{U}$ & 7.2 & 4.2 & 2.7 & $\mu \mathrm{g} / \mathrm{L}$ \\
\hline \multirow[t]{2}{*}{$\mathrm{X} 616-24 B$} & Dissolved $\mathrm{Cr}$ & 3.9 & 7.7 & 5.8 & 2.7 & $\mu \mathrm{g} / \mathrm{L}$ \\
\hline & Total $\mathrm{Cr}$ & 7.9 & 71.2 & 32.1 & 34.2 & $\mu \mathrm{g} / \mathrm{L}$ \\
\hline \multirow[t]{2}{*}{$\mathrm{X} 616-28 \mathrm{~B}$} & Dissolved $\mathrm{Cr}$ & $2.5 \mathrm{U}$ & $2.6 \mathrm{U}$ & NA & NA & $\mu \mathrm{g} / \mathrm{L}$ \\
\hline & Total $\mathrm{Cr}$ & $2.6 \mathrm{U}$ & 3.6 & 2.6 & 1.2 & $\mu \mathrm{g} / \mathrm{L}$ \\
\hline
\end{tabular}

${ }^{\circ}$ Qualifiers include: U-parameter was not detected; the result is the attainable detection limit for the sample.

NA -at least $50 \%$ of the results for the given parameter at this location were below detection; therefore, mean and standard deviation functions are not applicable.

${ }^{\mathrm{C}}$ Chromium. 
Table 4.9. Portsmouth groundwater monitoring for 1994: surface water ${ }^{a}$ monitoring results for volatile organic compounds

\begin{tabular}{|c|c|c|c|c|c|c|}
\hline Well & Parameter ${ }^{b}$ & $\operatorname{Min}^{c}$ & $\operatorname{Max}^{c}$ & $\operatorname{Mean}^{d}$ & $\begin{array}{c}\text { Standard } \\
\text { deviation }^{d}\end{array}$ & Units \\
\hline \multirow[t]{2}{*}{ BRC-SW01 } & $1,1,1-\mathrm{TCA}$ & $2 U$ & $2 U$ & NA & NA & $\mu \mathrm{g} / \mathrm{L}$ \\
\hline & TCE & $2 U$ & $2 \mathrm{U}$ & NA & NA & $\mu \mathrm{g} / \mathrm{L}$ \\
\hline \multirow[t]{2}{*}{ BRC-SW02 } & $1,1,1-\mathrm{TCA}$ & $2 \mathrm{U}$ & $2 \mathrm{U}$ & NA & NA & $\mu \mathrm{g} / \mathrm{L}$ \\
\hline & TCE & $2 U$ & $2 U$ & NA & NA & $\mu \mathrm{g} / \mathrm{L}$ \\
\hline \multirow[t]{2}{*}{ EDD-SW01 } & $1,1,1-\mathrm{TCA}$ & $2 \mathrm{U}$ & $2 \mathrm{U}$ & NA & NA & $\mu \mathrm{g} / \mathrm{L}$ \\
\hline & TCE & $2 U$ & 2 & NA & NA & $\mu \mathrm{g} / \mathrm{L}$ \\
\hline \multirow[t]{2}{*}{ LBC-SW01 } & $1,1,1-\mathrm{TCA}$ & $2 U$ & $2 U$ & NA & NA & $\mu \mathrm{g} / \mathrm{L}$ \\
\hline & TCE & $2 \mathrm{U}$ & 24 & NA & NA & $\mu \mathrm{g} / \mathrm{L}$ \\
\hline \multirow[t]{2}{*}{ LBC-SW02 } & $1,1,1-\mathrm{TCA}$ & $2 \mathrm{U}$ & $2 U$ & NA & NA & $\mu \mathrm{g} / \mathrm{L}$ \\
\hline & TCE & $2 \mathrm{U}$ & 18 & NA & NA & $\mu \mathrm{g} / \mathrm{L}$ \\
\hline \multirow[t]{2}{*}{ LBC-SW03 } & $1,1,1-\mathrm{TCA}$ & $2 U$ & $2 U$ & NA & NA & $\mu \mathrm{g} / \mathrm{L}$ \\
\hline & TCE & $2 \mathrm{U}$ & 7 & NA & NA & $\mu \mathrm{g} / \mathrm{L}$ \\
\hline \multirow[t]{2}{*}{ LBC-SW04 } & 1,1,1-TCA & $2 U$ & $2 \mathrm{U}$ & NA & NA & $\mu \mathrm{g} / \mathrm{L}$ \\
\hline & TCE & $2 U$ & 2 & NA & NA & $\mu \mathrm{g} / \mathrm{L}$ \\
\hline \multirow[t]{2}{*}{ NHP-SW01 } & $1,1,1-\mathrm{TCA}$ & $2 \mathrm{U}$ & $2 U$ & NA & NA & $\mu \mathrm{g} / \mathrm{L}$ \\
\hline & TCE & $2 U$ & $2 U$ & NA & NA & $\mu \mathrm{g} / \mathrm{L}$ \\
\hline \multirow[t]{2}{*}{ UND-SW01 } & $1,1,1-\mathrm{TCA}$ & $2 \mathrm{U}$ & $2 \mathrm{U}$ & NA & NA & $\mu \mathrm{g} / \mathrm{L}$ \\
\hline & TCE & $2 \mathrm{U}$ & 3 & 2 & 1 & $\mu \mathrm{g} / \mathrm{L}$ \\
\hline \multirow[t]{2}{*}{ UND-SW02 } & $1,1,1-\mathrm{TCA}$ & $2 U$ & $2 U$ & NA & $\mathrm{NA}$ & $\mu \mathrm{g} / \mathrm{L}$ \\
\hline & TCE & $2 U$ & $2 U$ & NA & NA & $\mu \mathrm{g} / \mathrm{L}$ \\
\hline \multirow[t]{2}{*}{ WDD-SW01 } & $1,1,1-\mathrm{TCA}$ & $2 U$ & $2 \mathrm{U}$ & NA & NA & $\mu \mathrm{g} / \mathrm{L}$ \\
\hline & TCE & $2 U$ & $2 U$ & NA & NA & $\mu \mathrm{g} / \mathrm{L}$ \\
\hline \multirow[t]{2}{*}{ WDD-SW02 } & $1,1,1-\mathrm{TCA}$ & $2 \mathrm{U}$ & $2 U$ & NA & NA & $\mu \mathrm{g} / \mathrm{L}$ \\
\hline & TCE & $2 U$ & $2 U$ & NA & NA & $\mu \mathrm{g} / \mathrm{L}$ \\
\hline \multirow[t]{2}{*}{ WDD-SW03 } & 1,1,1-TCA & $2 U$ & $2 \mathrm{U}$ & NA & NA & $\mu \mathrm{g} / \mathrm{L}$ \\
\hline & TCE & $2 \mathrm{U}$ & $2 \mathrm{U}$ & NA & NA & $\mu \mathrm{g} / \mathrm{L}$ \\
\hline
\end{tabular}

${ }^{a}$ Surface water samples are collected to analyze groundwater discharge.

${ }^{b}$ Parameter abbreviations: 1,1,1-TCA $=1,1,1$-trichloroethane; $\mathrm{TCE}=$ trichloroethylene.

'Qualifiers include U-parameter was not detected; the result is the attainable detection limit for the sample.

NA-at least $50 \%$ of the results for the given parameter at this location were below detection; therefore, mean and standard deviation functions are not applicable. 
Table 4.10. Portsmouth groundwater monitoring for 1994: surface water monitoring results for radionuclide parameters

\begin{tabular}{|c|c|c|c|c|c|c|}
\hline Well & Parameter & $\operatorname{Min}^{b}$ & $\operatorname{Max}^{b}$ & Mean $^{c}$ & $\begin{array}{l}\text { Standard } \\
\text { deviation }^{c}\end{array}$ & Units \\
\hline \multirow[t]{4}{*}{ BRC-SW01 } & Gross alpha & $6 \mathrm{U}$ & 13 & NA & NA & $\mathrm{pCi} / \mathrm{L}$ \\
\hline & Gross beta & 10 & 17 & 11.0 & 5.0 & $\mathrm{pCi} / \mathrm{L}$ \\
\hline & Technetium & $22 \mathrm{U}$ & $24 \mathrm{U}$ & NA & NA & $\mathrm{pCi} / \mathrm{L}$ \\
\hline & Total uranium & $1 \mathrm{U}$ & 3.7 & NA & NA & $\mu \mathrm{g} / \mathrm{L}$ \\
\hline \multirow[t]{4}{*}{ BRC-SW02 } & Gross alpha & $7 \mathrm{U}$ & 13 & NA & NA & $\mathrm{pCi} / \mathrm{L}$ \\
\hline & Gross beta & $10 U$ & $11 \mathrm{U}$ & NA & NA & $\mathrm{pCi} / \mathrm{L}$ \\
\hline & Technetium & $22 \mathrm{U}$ & $24 \mathrm{U}$ & NA & NA & $\mathrm{pCi} / \mathrm{L}$ \\
\hline & Total uranium & 1.2 & 1.9 & 1.3 & 0.5 & $\mu \mathrm{g} / \mathrm{L}$ \\
\hline \multirow[t]{4}{*}{ EDD-SW01 } & Gross alpha & $5 \mathrm{U}$ & 44 & 22.6 & 18.5 & $\mathrm{pCi} / \mathrm{L}$ \\
\hline & Gross beta & $9 U$ & 18 & $\mathrm{NA}$ & $\mathrm{NA}$ & $\mathrm{pCi} / \mathrm{L}$ \\
\hline & Technetium & $22 \mathrm{U}$ & 24 & $\mathrm{NA}$ & NA & $\mathrm{pCi} / \mathrm{L}$ \\
\hline & Total uranium & 1.1 & 7.3 & 4.1 & 2.6 & $\mu \mathrm{g} / \mathrm{L}$ \\
\hline \multirow[t]{4}{*}{ LBC-SW01 } & Gross alpha & $5 \mathrm{U}$ & 36 & NA & NA & $\mathrm{pCi} / \mathrm{L}$ \\
\hline & Gross beta & $9 \mathrm{U}$ & 16 & NA & NA & $\mathrm{pCi} / \mathrm{L}$ \\
\hline & Technetium & $22 \mathrm{U}$ & $24 \mathrm{U}$ & NA & $\mathrm{NA}$ & $\mathrm{pCi} / \mathrm{L}$ \\
\hline & Total uranium & $1 U$ & 4.9 & 2.1 & 2.0 & $\mu \mathrm{g} / \mathrm{L}$ \\
\hline \multirow[t]{4}{*}{ LBC-SW02 } & Gross alpha & $5 \mathrm{U}$ & 17 & 8.6 & 6.2 & $\mathrm{pCi} / \mathrm{L}$ \\
\hline & Gross beta & $9 \mathrm{U}$ & 11 & NA & NA & $\mathrm{pCi} / \mathrm{L}$ \\
\hline & Technetium & $22 \mathrm{U}$ & $24 \mathrm{U}$ & NA & $\mathrm{NA}$ & $\mathrm{pCi} / \mathrm{L}$ \\
\hline & Total uranium & $1 \mathrm{U}$ & 3.7 & 1.9 & 1.3 & $\mu \mathrm{g} / \mathrm{L}$ \\
\hline \multirow[t]{4}{*}{ LBC-SW03 } & Gross alpha & $5 \mathrm{U}$ & 44 & NA & $\mathrm{NA}$ & $\mathrm{pCi} / \mathrm{L}$ \\
\hline & Gross beta & $9 \mathrm{U}$ & 16 & NA & NA & $\mathrm{pCi} / \mathrm{L}$ \\
\hline & Technetium & $22 \mathrm{U}$ & $24 \mathrm{U}$ & NA & $\mathrm{NA}$ & $\mathrm{pCi} / \mathrm{L}$ \\
\hline & Total uranium & $1 \mathrm{U}$ & 4.9 & 2.1 & 1.9 & $\mu \mathrm{g} / \mathrm{L}$ \\
\hline \multirow[t]{4}{*}{ LBC-SW04 } & Gross alpha & $5 \mathrm{U}$ & 29 & $\mathrm{NA}$ & NA & $\mathrm{pCi} / \mathrm{L}$ \\
\hline & Gross beta & $9 \mathrm{U}$ & 19 & NA & NA & $\mathrm{pCi} / \mathrm{L}$ \\
\hline & Technetium & $22 \mathrm{U}$ & $24 \mathrm{U}$ & NA & NA & $\mathrm{pCi} / \mathrm{L}$ \\
\hline & Total uranium & $1 \mathrm{U}$ & 5.5 & 2.9 & 2.3 & $\mu \mathrm{g} / \mathrm{L}$ \\
\hline \multirow[t]{4}{*}{ NHP-SW01 } & Gross alpha & $5 \mathrm{U}$ & 13 & NA & NA & $\mathrm{pCi} / \mathrm{L}$ \\
\hline & Gross beta & $9 \mathrm{U}$ & $10 \mathrm{U}$ & NA & NA & $\mathrm{pC} / / \mathrm{L}$ \\
\hline & Technetium & $22 \mathrm{U}$ & $24 \mathrm{U}$ & NA & NA & $\mathrm{pCi} / \mathrm{L}$ \\
\hline & Total uranium & 2.9 & 7.9 & 5.9 & 2.1 & $\mu \mathrm{g} / \mathrm{L}$ \\
\hline \multirow[t]{4}{*}{ UND-SW01 } & Gross alpha & $5 \mathrm{U}$ & $7 \mathrm{U}$ & NA & NA & $\mathrm{pCi} / \mathrm{L}$ \\
\hline & Gross beta & $9 \mathrm{U}$ & $11 \mathrm{U}$ & NA & NA & $\mathrm{pCi} / \mathrm{L}$ \\
\hline & Technetium & $22 \mathrm{U}$ & $24 U$ & $\mathrm{NA}$ & $\mathrm{NA}$ & $\mathrm{pCi} / \mathrm{L}$ \\
\hline & Total uranium & $1 \mathrm{U}$ & 2.1 & 1.7 & 0.8 & $\mu \mathrm{g} / \mathrm{L}$ \\
\hline
\end{tabular}


Table 4.10 (continued)

\begin{tabular}{llccccc}
\hline Well & Parameter & Min $^{b}$ & Max $^{b}$ & Mean $^{c}$ & $\begin{array}{c}\text { Standard } \\
\text { deviation }^{c}\end{array}$ & Units \\
\hline UND-SW02 & Gross alpha & $5 \mathrm{U}$ & $6 \mathrm{U}$ & $\mathrm{NA}$ & $\mathrm{NA}$ & $\mathrm{pCi} / \mathrm{L}$ \\
& Gross beta & $9 \mathrm{U}$ & 11 & $\mathrm{NA}$ & $\mathrm{NA}$ & $\mathrm{pCi} / \mathrm{L}$ \\
& Technetium & $22 \mathrm{U}$ & $24 \mathrm{U}$ & $\mathrm{NA}$ & $\mathrm{NA}$ & $\mathrm{pCi} / \mathrm{L}$ \\
& Total uranium & $1 \mathrm{U}$ & 2.3 & $\mathrm{NA}$ & $\mathrm{NA}$ & $\mu \mathrm{g} / \mathrm{L}$ \\
WDD-SW01 & Gross alpha & $5 \mathrm{U}$ & 16 & $\mathrm{NA}$ & $\mathrm{NA}$ & $\mathrm{pCi} / \mathrm{L}$ \\
& Gross beta & $9 \mathrm{U}$ & 14 & $\mathrm{NA}$ & $\mathrm{NA}$ & $\mathrm{pCi} / \mathrm{L}$ \\
& Technetium & $22 \mathrm{U}$ & $22 \mathrm{U}$ & $\mathrm{NA}$ & $\mathrm{NA}$ & $\mathrm{pCi} / \mathrm{L}$ \\
& Total uranium & 1.8 & 7.4 & 4.8 & 2.4 & $\mu \mathrm{g} / \mathrm{L}$ \\
WDD-SW02 & Gross alpha & $6 \mathrm{U}$ & $12 \mathrm{U}$ & $\mathrm{NA}$ & $\mathrm{NA}$ & $\mathrm{pCi} / \mathrm{L}$ \\
& Gross beta & $9 \mathrm{U}$ & $11 \mathrm{U}$ & $\mathrm{NA}$ & $\mathrm{NA}$ & $\mathrm{pCi} / \mathrm{L}$ \\
& Technetium & $22 \mathrm{U}$ & $24 \mathrm{U}$ & $\mathrm{NA}$ & $\mathrm{NA}$ & $\mathrm{pCi} / \mathrm{L}$ \\
& Total uranium & $1 \mathrm{U}$ & 2.8 & 1.8 & 1.2 & $\mu \mathrm{g} / \mathrm{L}$ \\
WDD-SW03 & Gross alpha & $5 \mathrm{U}$ & $8 \mathrm{U}$ & $\mathrm{NA}$ & $\mathrm{NA}$ & $\mathrm{pCi} / \mathrm{L}$ \\
& Gross beta & $9 \mathrm{U}$ & $11 \mathrm{U}$ & $\mathrm{NA}$ & $\mathrm{NA}$ & $\mathrm{pCi} / \mathrm{L}$ \\
& Technetium & $22 \mathrm{U}$ & $24 \mathrm{U}$ & $\mathrm{NA}$ & $\mathrm{NA}$ & $\mathrm{pCi} / \mathrm{L}$ \\
& Total uranium & 3.0 & 4.8 & 3.6 & 0.8 & $\mu \mathrm{g} / \mathrm{L}$ \\
\hline
\end{tabular}

${ }^{a}$ Surface water samples are collected to analyze groundwater discharge.

${ }^{b}$ Qualifiers include: U-parameter was not detected; the result is the attainable detection limit for the sample.

NA -at least $50 \%$ of the results for the given parameter at this location were below detection; therefore, mean and standard deviation functions are not applicable. 
Table 4.11. Portsmouth groundwater monitoring for 1994: results for trichloroethylene at $\mathrm{X}-701 \mathrm{C}$

\begin{tabular}{ccccccc}
\hline Well & Parameter & Min & Max & Mean & $\begin{array}{c}\text { Standard } \\
\text { deviation }\end{array}$ & Units \\
\hline X701-68G & Trichloroethylene & 30 & 46 & 40 & 6.8 & $\mu \mathrm{g} / \mathrm{L}$ \\
X701-69G & Trichloroethylene & $300 \mathrm{~J}^{a}$ & 2690 & 1663 & 997 & $\mu \mathrm{g} / \mathrm{L}$ \\
X701-70G & Trichloroethylene & 170 & 470 & 310 & 124 & $\mu \mathrm{g} / \mathrm{L}$ \\
\hline
\end{tabular}

${ }^{a} \mathrm{~J}=$ data qualifier indicating that the result must be considered an estimate.

Table 4.12. Portsmouth groundwater monitoring for 1994: results for radionuclide parameters at X-701C

\begin{tabular}{|c|c|c|c|c|c|c|}
\hline Well & Parameter & $\operatorname{Min}^{a}$ & $\operatorname{Max}^{a}$ & $\operatorname{Mean}^{b}$ & $\begin{array}{c}\text { Standard } \\
\text { deviation }^{b}\end{array}$ & Units \\
\hline \multirow[t]{4}{*}{ X701-68G } & Gross alpha & $12 \mathrm{U}$ & $14 \mathrm{U}$ & NA & NA & $\mathrm{pCi} / \mathrm{L}$ \\
\hline & Gross beta & 14 & 18 & 16 & 1.6 & $\mathrm{pCi} / \mathrm{L}$ \\
\hline & Technetium & $22 \mathrm{U}$ & 26 & NA & NA & $\mathrm{pCi} / \mathrm{L}$ \\
\hline & Total uranium & $1.0 \mathrm{U}$ & 2.1 & NA & NA & $\mu \mathrm{g} / \mathrm{L}$ \\
\hline \multirow[t]{4}{*}{$\mathrm{X701-69G}$} & Gross alpha & $3 \mathrm{U}$ & $20 \mathrm{U}$ & NA & NA & $\mathrm{pCi} / \mathrm{L}$ \\
\hline & Gross beta & $10 \mathrm{U}$ & $20 \mathrm{U}$ & NA & NA & $\mathrm{pCi} / \mathrm{L}$ \\
\hline & Technetium & $22 \mathrm{U}$ & $22 \mathrm{U}$ & NA & NA & $\mathrm{pCi} / \mathrm{L}$ \\
\hline & Total uranium & 6.2 & 7.0 & 6.5 & 0.4 & $\mu g / L$ \\
\hline \multirow[t]{4}{*}{ X701-70G } & Gross alpha & $6 \mathrm{U}$ & $9 \mathrm{U}$ & NA & NA & $\mathrm{pCi} / \mathrm{L}$ \\
\hline & Gross beta & 42 & 49 & 45 & 3.2 & $\mathrm{pCi} / \mathrm{L}$ \\
\hline & Technetium & 51 & 71 & 61 & 9.7 & $\mathrm{pCi} / \mathrm{L}$ \\
\hline & Total uranium & 3.0 & 3.4 & 3.1 & 0.2 & $\mu \mathrm{g} / \mathrm{L}$ \\
\hline
\end{tabular}

${ }^{a}$ Qualifiers include U-parameter was not detected; the result is the attainable detection limit for the sample.

NA-at least $50 \%$ of the results for the given parameter at this location were below detection; therefore, mean and standard deviation functions are not applicable. 